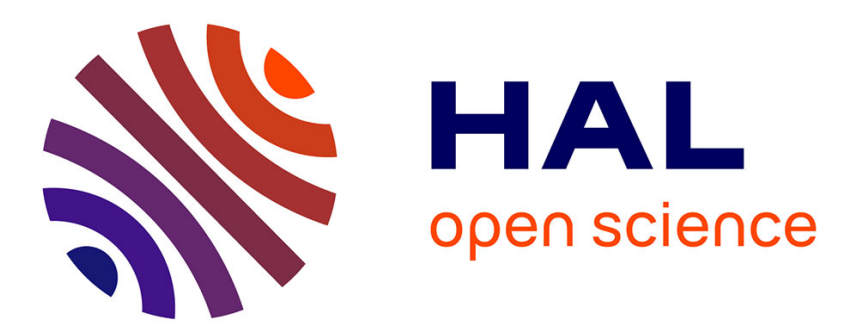

\title{
Utilité clinique de la TEP cérébrale à la 18F-FDOPA dans la prise en charge des syndromes parkinsoniens
}

\author{
Aurélie Schiazza
}

\section{To cite this version:}

Aurélie Schiazza. Utilité clinique de la TEP cérébrale à la 18F-FDOPA dans la prise en charge des syndromes parkinsoniens. Médecine humaine et pathologie. 2015. dumas-01286120

\section{HAL Id: dumas-01286120 https://dumas.ccsd.cnrs.fr/dumas-01286120}

Submitted on 10 Mar 2016

HAL is a multi-disciplinary open access archive for the deposit and dissemination of scientific research documents, whether they are published or not. The documents may come from teaching and research institutions in France or abroad, or from public or private research centers.
L'archive ouverte pluridisciplinaire HAL, est destinée au dépôt et à la diffusion de documents scientifiques de niveau recherche, publiés ou non, émanant des établissements d'enseignement et de recherche français ou étrangers, des laboratoires publics ou privés. 
UNIVERSITÉ DE NICE SOPHIA ANTIPOLIS

FACULTÉ DE MÉDECINE DE NICE

\section{UTILITÉ CLINIQUE DE LA TEP CÉRÉBRALE À LA 18F-FDOPA DANS LA} PRISE EN CHARGE DES SYNDROMES PARKINSONIENS

\section{THÈSE D’EXERCICE EN MÉDECINE}

Présentée et soutenue publiquement le vendredi 25 septembre 2015

Pour obtenir le grade de Docteur en Médecine

Par Aurélie SCHIAZZA 
UNIVERSITÉ DE NICE SOPHIA ANTIPOLIS

FACULTÉ DE MÉDECINE DE NICE

\section{«UTILITÉ CLINIQUE DE LA TEP CÉRÉBRALE À LA 18F-FDOPA DANS LA PRISE EN CHARGE DES SYNDROMES PARKINSONIENS »}

\section{THÈSE D’EXERCICE EN MÉDECINE}

Présentée et soutenue publiquement le vendredi 25 septembre 2015

Pour obtenir le grade de Docteur en Médecine

Par Aurélie SCHIAZZA

Née le 29/04/1987 à NICE

COMPOSITION DU JURY

Président du Jury: $\quad$ Monsieur le Professeur DARCOURT Jacques

Directeur de Thèse : Monsieur le Professeur DARCOURT Jacques

Membres du Jury: Monsieur le Professeur PADOVANI Bernard

Monsieur le Professeur THOMAS Pierre

Monsieur le Docteur BORG Michel

Monsieur le Docteur FONTANA Xavier

Monsieur le Docteur TIFRATENE Karim 


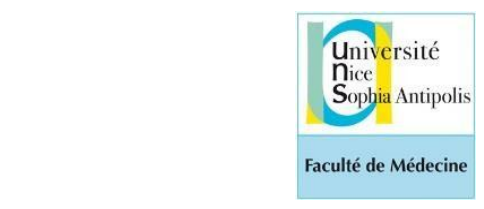

UNIVERSITÉ NICE-SOPHIAANTIPOLIS

FACULTÉ DE MÉDECINE

Liste des professeurs au 1er septembre 2015 à la Faculté de Médecine de Nice

Doyen

Vice-Doyen

Assesseurs

Conservateur de la bibliothèque

Directrice administrative des services

Doyens Honoraires
M. BAQUÉ Patrick

M. BOILEAU Pascal

M. ESNAULT Vincent

M. CARLES Michel

Mme BREUIL Véronique

M. MARTY Pierre

Mme DE LEMOS Annelyse

Mme CALLEA Isabelle

M. AYRAUD Noël

M. RAMPAL Patrick

M. BENCHIMOL Daniel

\section{Professeurs Honoraires}

M. BALAS Daniel

M. BATT Michel M.

BLAIVE Bruno

M. BOQUET Patrice

M. BOURGEON André

M. BOUTTÉ Patrick

M. BRUNETON Jean-Noël

Mme BUSSIERE Françoise

M. CAMOUS Jean-Pierre M.

CHATEL Marcel

M. COUSSEMENT Alain

M. DARCOURT Guy

M. DELLAMONICA Pierre

M. DELMONT Jean

M. DEMARD François

M. DOLISI Claude

M. FRANCO Alain

M. FREYCHET Pierre

M. GÉRARD Jean-Pierre

M. GILLET Jean-Yves

M. GRELLIER Patrick

M. HARTER Michel

M. INGLESAKIS Jean-André
M. LALANNE Claude-Michel

M. LAMBERT Jean-Claude

M. LAZDUNSKI Michel

M. LEFEBVRE Jean-Claude

M. LE BAS Pierre

M. LE FICHOUX Yves

Mme LEBRETON Elisabeth

M. LOUBIERE Robert

M. MARIANI Roger

M. MASSEYEFF René

M. MATTEI Mathieu

M. MOUIEL Jean

Mme MYQUEL Martine

M. OLLIER Amédée

M. ORTONNE Jean-Paul

M. SAUTRON Jean Baptiste

M. SCHNEIDER Maurice

M. SERRES Jean-Jacques

M. TOUBOL Jacques

M. TRAN Dinh Khiem

M VAN OBBERGHEN Emmanuel

M.ZIEGLER Gérard 


\section{M.C.A. Honoraire}

M.C.U. Honoraires
Mlle ALLINE Madeleine
M. ARNOLD Jacques
M. BASTERIS Bernard
Mlle CHICHMANIAN Rose-Marie
Mme DONZEAU Michèle
M. EMILIOZZI Roméo M.
FRANKEN Philippe M.
GASTAUD Marcel
M.GIRARD-PIPAU Fernand
M. GIUDICELLI Jean
M. MAGNÉ Jacques Mme
MEMRAN Nadine M.
MENGUAL Raymond M.
POIRÉE Jean-Claude
Mme ROURE Marie-Claire

\section{PROFESSEURS CLASSE EXCEPTIONNELLE}

$\begin{array}{ll}\text { M. } & \text { AMIEL Jean } \\ \text { M. } & \text { BENCHIMOL Daniel } \\ \text { M. } & \text { BOILEAU Pascal } \\ \text { M. } & \text { DARCOURT Jacques } \\ \text { M. } & \text { DESNUELLE Claude } \\ \text { Mme } & \text { EULLER-ZIEGLER Liana } \\ \text { M. } & \text { FENICHEL Patrick } \\ \text { M. } & \text { FUZIBET Jean-Gabriel } \\ \text { M. } & \text { GASTAUD Pierre } \\ \text { M. } & \text { GILSON Éric } \\ \text { M. } & \text { GRIMAUD Dominique } \\ \text { M. } & \text { HASSEN KHODJA Reda } \\ \text { M. } & \text { HÉBUTERNE Xavier } \\ \text { M. } & \text { HOFMAN Paul } \\ \text { M. } & \text { LACOUR Jean-Philippe } \\ \text { M. } & \text { MARTY Pierre } \\ \text { M. } & \text { MICHIELS Jean-François } \\ \text { M. } & \text { MOUROUX Jérôme } \\ \text { M. } & \text { PAQUIS Philippe } \\ \text { M. } & \text { PRINGUEY Dominique } \\ \text { M. } & \text { QUATREHOMME Gérald } \\ \text { M. } & \text { M.ROBERT Philippe } \\ \text { M. } & \text { SANTINI Joseph } \\ \text { M. } & \text { THYSS Antoine } \\ & \end{array}$

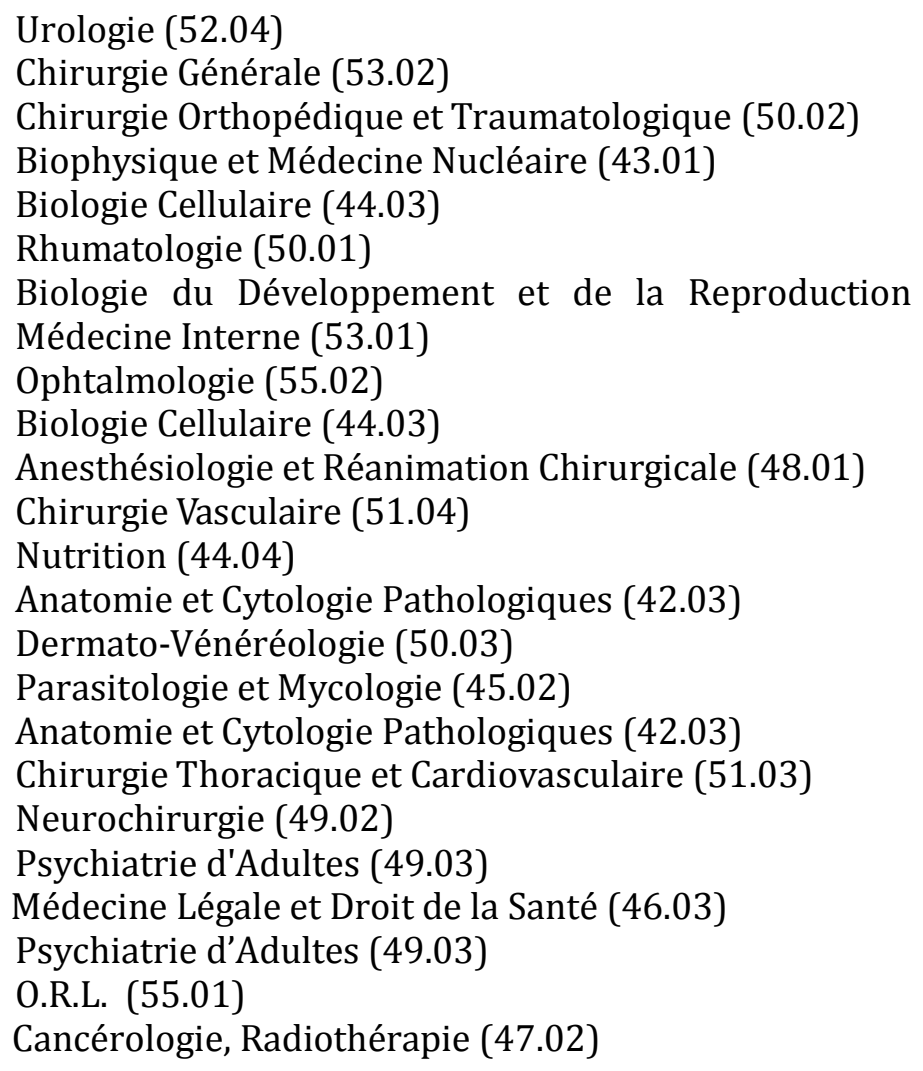




\section{PROFESSEURS PREMIERE CLASSE}

\begin{tabular}{|c|c|}
\hline Mme & ASKENAZY-GITTARD Florence \\
\hline M. & BAQUÉ Patrick \\
\hline M. & BÉRARD Étienne \\
\hline M. & BERNARDIN Gilles \\
\hline M. & BONGAIN André \\
\hline M. & CASTILLO Laurent \\
\hline Mme & CRENESSE Dominique \\
\hline M. & DE PERETTI Fernand \\
\hline M. & DRICI Milou-Daniel \\
\hline M. & ESNAULT Vincent \\
\hline M. & FERRARI Émile \\
\hline M. & FERRERO Jean-Marc \\
\hline M. & GIBELIN Pierre \\
\hline M. & GUGENHEIM Jean \\
\hline Mme & ICHAI Carole \\
\hline M. & LONJON Michel \\
\hline M. & MARQUETTE Charles-Hugo \\
\hline M. & MOUNIER Nicolas \\
\hline M. & PADOVANI Bernard \\
\hline Mme & PAQUIS Véronique \\
\hline M. & PRADIER Christian \\
\hline M. & RAUCOULES-AIMÉ Marc \\
\hline Mme & RAYNAUD Dominique \\
\hline M. & ROSENTHAL Éric \\
\hline M. & SCHNEIDER Stéphane \\
\hline M. & STACCINI Pascal \\
\hline M. & THOMAS Pierre \\
\hline M. & TRAN Albert \\
\hline
\end{tabular}

Pédopsychiatrie (49.04)

Anatomie - Chirurgie Générale (42.01)

Pédiatrie (54.01)

Réanimation Médicale (48.02)

Gynécologie-Obstétrique (54.03)

O.R.L. (55.01)

Physiologie (44.02)

Anatomie-Chirurgie Orthopédique (42.01)

Pharmacologie Clinique (48.03)

Néphrologie (52-03)

Cardiologie (51.02)

Cancérologie ; Radiothérapie (47.02)

Cardiologie (51.02)

Chirurgie Digestive (52.02)

Anesthésiologie et Réanimation Chirurgicale (48.01)

Neurochirurgie (49.02)

Pneumologie (51.01)

Cancérologie, Radiothérapie (47.02)

Radiologie et Imagerie Médicale (43.02)

Génétique (47.04)

Épidémiologie, Économie de la Santé et Prévention

Anesthésie et Réanimation Chirurgicale (48.01)

Hématologie (47.01)

Médecine Interne (53.01)

Nutrition (44.04)

Biostatistiques et Informatique Médicale (46.04)

Neurologie (49.01)

Hépato Gastro-entérologie (52.01)

\section{PROFESSEURS DEUXIEME CLASSE}

$\begin{array}{ll}\text { M. } & \text { ALBERTINI Marc } \\ \text { Mme } & \text { BAILLIF Stéphanie } \\ \text { M. } & \text { BAHADORAN Philippe } \\ \text { M. } & \text { BARRANGER Emmanuel } \\ \text { M. } & \text { BENIZRI Emmanuel } \\ \text { M. } & \text { BENOIT Michel } \\ \text { Mme } & \text { BLANC-PEDEUTOUR Florence } \\ \text { M. } & \text { BREAUD Jean } \\ \text { Mlle } & \text { BREUIL Véronique } \\ \text { M. } & \text { CANIVET Bertrand } \\ \text { M. } & \text { CARLES Michel } \\ \text { M. } & \text { CASSUTO Jill-Patrice } \\ \text { M. } & \text { CHEVALLIER Patrick } \\ \text { Mme } & \text { CHINETTI Giulia } \\ \text { M. } & \text { DELOTTE Jérôme } \\ \text { M. } & \text { DUMONTIER Christian } \\ \text { M. } & \text { FONTAINE Denys } \\ \text { M. } & \text { FOURNIER Jean-Paul } \\ \text { M. } & \text { FREDENRICH Alexandre } \\ \text { Mlle } & \text { GIORDANENGO Valérie } \\ \text { M. } & \text { GUÉRIN Olivier } \\ \text { M. } & \text { HANNOUN-LEVI Jean-Michel }\end{array}$

Pédiatrie (54.01)

Ophtalmologie (55.02)

Cytologie et Histologie (42.02)

Gynécologie Obstétrique (54.03)

Chirurgie Générale (53.02)

Psychiatrie (49.03)

Cancérologie - Génétique (47.02)

Chirurgie Infantile (54-02)

Rhumatologie (50.01)

Médecine Interne (53.01)

Anesthésiologie Réanimation (48.01)

Hématologie et Transfusion (47.01)

Radiologie et Imagerie Médicale (43.02)

Biochimie-Biologie Moléculaire (44.01)

Gynécologie-obstétrique (54.03)

Chirurgie plastique

Neurochirurgie (49.02)

Thérapeutique (48-04)

Endocrinologie, Diabète et Maladies métaboliques

Bactériologie-Virologie (45.01)

Gériatrie (48.04)

Cancérologie ; Radiothérapie (47.02) 


\section{PROFESSEURS DEUXIEME CLASSE (suite)}

$\begin{array}{ll}\text { M. } & \text { IANNELLI Antonio } \\ \text { M } & \text { JEAN BAPTISTE Elixène } \\ \text { M. } & \text { JOURDAN Jacques } \\ \text { M. } & \text { LEVRAUT Jacques } \\ \text { M. } & \text { PASSERON Thierry } \\ \text { M. } & \text { PICHE Thierry } \\ \text { M. } & \text { ROGER Pierre-Marie } \\ \text { M. } & \text { ROHRLICH Pierre } \\ \text { M. } & \text { RUIMY Raymond } \\ \text { Mme } & \text { SACCONI Sabrina } \\ \text { M. } & \text { SADOUL Jean-Louis } \\ \text { M. } & \text { TROJANI Christophe } \\ \text { M. } & \text { VENISSAC Nicolas }\end{array}$

Chirurgie Digestive (52.02)

Chirurgie vasculaire (51.04)

Chirurgie Thoracique et Cardiovasculaire (51.03)

Anesthésiologie et Réanimation Chirurgicale (48.01)

Dermato-Vénéréologie (50-03)

Gastro-entérologie (52.01)

Maladies Infectieuses ; Maladies Tropicales (45.03)

Pédiatrie (54.01)

Bactériologie-virologie (45.01)

Neurologie (49.01)

Endocrinologie, Diabète et Maladies Métaboliques

Chirurgie Orthopédique et Traumatologique (50.02)

Chirurgie Thoracique et Cardiovasculaire (51.03)

\section{PROFESSEUR DES UNIVERSITÉS}

M. HOFLIGER Philippe Médecine Générale

PROFESSEUR AGRÉGÉ

Mme LANDI Rebecca Anglais

Mme ROSE Anglais

\section{MAITRES DE CONFÉRENCES DES UNIVERSITÉS - PRATICIENS HOSPITALIERS}

$\begin{array}{ll}\text { Mme } & \text { ALUNNI Véronique } \\ \text { M. } & \text { AMBROSETTI Damien } \\ \text { Mme } & \text { BANNWARTH Sylvie } \\ \text { M. } & \text { BENOLIEL José } \\ \text { Mme } & \text { BERNARD-POMIER Ghislaine } \\ \text { Mme } & \text { BUREL-VANDENBOS Fanny } \\ \text { M. } & \text { DOGLIO Alain } \\ \text { M } & \text { DOYEN Jérôme } \\ \text { M } & \text { FAVRE Guillaume } \\ \text { M. } & \text { FOSSE Thierry } \\ \text { M. } & \text { GARRAFFO Rodolphe } \\ \text { Mme } & \text { GIOVANNINI-CHAMI Lisa } \\ \text { Mme } & \text { HINAULT Charlotte } \\ \text { Mme } & \text { LEGROS Laurence } \\ \text { Mme } & \text { MAGNIÉ Marie-Noëlle } \\ \text { Mme } & \text { MOCERI Pamela } \\ \text { Mme } & \text { MUSSO-LASSALLE Sandra } \\ \text { M. } & \text { NAÏMI Mourad } \\ \text { M. } & \text { PHILIP Patrick } \\ \text { Mme } & \text { POMARES Christelle } \\ \text { M. } & \text { ROUX Christian } \\ \text { M. } & \text { TESTA Jean } \\ \text { M. } & \text { TOULON Pierre }\end{array}$

Médecine Légale et Droit de la Santé (46.03)

Cytologie et Histologie (42.02)

Génétique (47.04)

Biophysique et Médecine Nucléaire (43.01)

Immunologie (47.03)

Anatomie et Cytologie pathologiques (42.03)

Bactériologie-Virologie (45.01)

Radiothérapie (47.02)

Néphrologie (52.03)

Bactériologie-Virologie-Hygiène (45.01)

Pharmacologie Fondamentale (48.03)

Pédiatrie (54.01)

Biochimie et biologie moléculaire (44.01)

Hématologie et Transfusion (47.01)

Physiologie (44.02)

Cardiologie (51.02)

Anatomie et Cytologie pathologiques (42.03)

Biochimie et Biologie moléculaire (44.01)

Cytologie et Histologie (42.02)

Parasitologie et mycologie (45.02)

Rhumatologie (50.01)

Épidémiologie Économie de la Santé et Prévention

Hématologie et Transfusion (47.01) 


\section{PROFESSEURS ASSOCIÉS}

M BALDIN Jean-Luc

Médecine Générale

M COYNE John

M. GARDON Gilles

Anatomie et Cytologie

Mme PACZESNY Sophie

Médecine Générale

Hématologie (47.01)

\section{MAITRES DE CONFÉRENCES ASSOCIÉS}

M. DARMON David

Médecine Générale

Mme MONNIER Brigitte

Médecine Générale

\section{PROFESSEURS CONVENTIONNÉS DE L'UNIVERSITÉ}

M. BERTRAND François

M. BROCKER Patrice

M. CHEVALLIER Daniel

Mme FOURNIER-MEHOUAS Manuella

M. JAMBOU Patrick

M. QUARANTA Jean-François
Médecine Interne

Médecine Interne Option Gériatrie Urologie

Médecine Physique et Réadaptation Coordination prélèvements Santé Publique 
«Ne va pas penser que, si une chose est difficile à comprendre pour toi, elle est incompréhensible pour tout homme; mais si une chose est possible et familière à un homme, crois bien aussi que tu peux l'atteindre » Marc Aurèle 
A mon grand-père Gabriel, A toi qui me manque tellement, A toi que j'aime éperdument, Je te dédie ce travail. 


\section{REMERCIEMENTS}

A Monsieur le Professeur Jacques DARCOURT

Vous avez d'abord été mon professeur sur les bancs de la Faculté de Nice puis ensuite mon maître tout au long de l'internat. Merci de m'avoir accueillie et initiée à la médecine nucléaire. Je vous exprime mon profond respect et toute ma gratitude pour tout ce que vous m'avez appris pendant mon internat, pour votre expérience, le goût de l'excellence et de la réflexion ainsi que vos conseils bienveillants. Merci infiniment de m'avoir permis de réaliser ce travail si intéressant et enrichissant et d'avoir partagé avec moi votre domaine d'expertise. Je suis très honorée que vous soyez ce soir le Président de ma thèse.

\section{A Monsieur le Professeur Bernard PADOVANI}

J'ai eu la chance de vous avoir comme maître de stage de Radiologie et je vous remercie pour ce que vous m'avez enseigné. Vous faites partie des personnes qui ont beaucoup compté dans ma formation médicale depuis les bancs de la Faculté. Je suis très honorée que vous ayez accepté de faire partie de mon Jury de thèse et vous en remercie.

\section{A Monsieur le Professeur Pierre THOMAS}

Vous me faites l'honneur de participer à ce jury. Je vous suis très reconnaissante d'évaluer ce travail et vous remercie de nous faire bénéficier de votre niveau d'expertise dans la Neurologie qui est une des spécialités médicales les plus pointues à mes yeux avec de grandes perspectives d'avenir, en collaboration avec la médecine nucléaire, je l'espère.

A Monsieur le Docteur Michel BORG

Merci pour votre expertise et votre niveau d'excellence. Je tiens tout particulièrement à vous remercier pour votre accompagnement et votre disponibilité qui m’ont aidée à initier cette étude. Vos conseils expérimentés m’ont guidée pour faire qu'aujourd'hui je suis face à vous pour le présenter. Sachez que je suis sensible à votre participation à l'estimation de ce travail.

\section{A Monsieur le Docteur Xavier FONTANA}

J'ai eu la chance d'être à tes côtés aux prémices de ma vie de médecin alors que ce fut pour toi les dernières années de ta carrière. C'était un plaisir pour moi d'être ton interne, tu m'as appris à travailler efficacement et toujours dans la bonne humeur. Je te remercie sincèrement d'avoir accepté de juger ce travail et je pense que tous les deux nous nous souviendrons longtemps de cette journée du vendredi 25 septembre 2015. 
A Monsieur le Docteur Karim TIFRATENE

Quel bonheur de te rencontrer, tu as su me donner l'élan dont j'avais besoin pour débuter ce travail, tes encouragements m'ont permis d'arriver à le réaliser et je te remercie infiniment pour ton aide si précieuse. Merci pour ta disponibilité, pour ton savoir et ta bienveillance. Je te suis profondément reconnaissante de me faire l'honneur de faire partie de ce jury et j'espère que nous aurons l'occasion de travailler encore ensemble dans le futur. 
A mes maîtres et condisciples :

A Docteur Matthieu-John OUVRIER, tu étais là quand j'ai fait mes premiers pas en médecine nucléaire, merci pour la générosité avec laquelle tu m'as accueillie dans le service puis formée à la médecine nucléaire. C'est un plaisir de travailler avec toi.

$\mathrm{Au}$ Docteur Danièle BENISVY, je te remercie sincèrement pour tout ce que tu m'as transmis, ta disponibilité et bien sûr tout le soutien et les bons conseils que tu m'as donnés ces derniers mois. J'espère avoir la chance de parfaire ma formation à tes côtés, tu es mon modèle!

$\mathrm{Au}$ Docteur Colette ZWARTHOED, tu m'as guidée lors de mon arrivée en médecine nucléaire. Je ne sais pas si tu réalises à quel point tu as joué un rôle important dans mon internat et ma formation. Je t'admire pour le médecin que tu es devenue, et te remercie d'être à mes côtés !

Au Docteur Micheline RAZZOUK-CADET,

Merci de m'avoir si gentiment accueillie pendant mes six mois à l'Archet. Merci pour votre enseignement ce fut un plaisir d'être votre interne tant sur le plan humain que professionnel et j'espère avoir la chance de pouvoir encore travailler avec vous dans les années à venir.

$\mathrm{Au}$ Docteur Philippe VIAU, merci de m'avoir transmis tes connaissances, en particulier en scintigraphie cardiaque. J'ai tellement apprécié travailler à tes côtés, toujours dans la bonne humeur. Merci pour ton énergie et ton accompagnement bienveillant pendant mon stage.

Au Docteur Renaud GUIGNARD, tu es aussi à l'origine de ce travail et sans ton départ tu m'aurais probablement aidé à le réaliser. Tu me manques beaucoup! Je te remercie sincèrement pour tout ce que tu m'as apporté au début de mon internat.

A Monsieur le Professeur Antoine THYSS, qui m'a accueillie à bras ouverts pour réaliser le DESC d'oncologie.

A mes chefs de la Réunion. Merci Cédric pour tout ce que tu m'as appris, ce fut un plaisir pour moi de travailler avec toi, d'apprendre de ton expertise. Merci David pour m'avoir si bien accueillie et pour m'avoir transmis ton savoir. J'ai passé 6 mois extraordinaires à la Réunion et c'est en grande partie grâce à vous.

A tous les Radiothérapeutes de Mougins qui m'ont si accueillie à bras ouverts. Vous m'avez ouvert au domaine de la Radiothérapie et je vous en remercie.

A Michael, Jean-Philippe et Philippe. Merci pour votre accueil dans votre service et pour m'avoir permis de faire de la médecine nucléaire lorsque j'étais en stage à côté de chez vous. Merci pour votre confiance et votre dynamisme. 
A Lydiane MONDOT, Sabrina MACARIO, Marie BAQUE et Stéphane CHANALET qui m'ont formée pendant 6 mois à la radiologie, ce fut un plaisir d'être à vos côtés et je vous remercie pour la bienveillance, la patience et la pédagogie dont vous avez fait preuve à mon égard.

A tous mes co-internes, car sans les co-internes l'internat ne serait pas l'internat ! On partage nos joies, nos peines, nos difficultés ensemble, merci pour votre soutien !:

A Julien et Alexandra de la Radio,

A Audrey, Alexander et tous les autres internes de cancéro et radiothérapie,

A Maxime, Marie et Romain de la médecine nucléaire qui je l'espère seront mes futurs collègues!

A toute l'équipe du CAL :

A Anne notre chef, merci pour ta gentillesse envers moi et ton soutien.

A tous les manipulateurs qui sont indispensables à l'exercice de notre métier: Pauline, Jérémy, Linda, Pierre, Romain, Elodie, Ingrid, Julie et Karima (et ceux que j'oublie maladroitement).

A Annie et bien sûr à toutes nos secrétaires qui font un travail indispensable avec une dédicace spéciale à Véronique et Faustine sans qui ce travail n'aurait pas vu le jour! Je vous suis infiniment reconnaissante pour votre aide.

A toutes les équipes de la Radio de Pasteur et St-Roch, de la médecine nucléaire de l'Archet et aussi de la Radiothérapie de Mougins avec une spéciale dédicace à Maeva et Carine. 


\section{A ma famille :}

A mes parents, ces quelques mots ne suffiront pas à vous dire tout l'amour, le respect et l'admiration que j'ai pour vous. Votre soutien a été le pilier de ma réussite. A mon père, merci Papa pour ton amour puissance dix millions. Notre complicité c'est notre force, tu es mon pilier. A tes côtés je me suis toujours sentie invincible, tu m'as tout appris, tu as fait révéler le meilleur de moi pour me permettre d'avancer. Tu es le papa idéal dont rêve toute fille, je t'aime.

A ma mère, merci Maman, ton niveau d'exigence m'a poussée vers le haut, tu m'as appris à ne pas avoir de limites, tu m'as dit que tout était à ma portée, tu m'as soutenu de tout ton cœur pour que je réussisse, tu as toujours su me faire oublier mes doutes et me donner confiance, tu m'as transmis ton assurance et ton humilité. J'espère un jour devenir une aussi bonne mère que toi. Tu es mon modèle, je t'aime.

A Ludo mon Amour, ma moitié pour la vie entière; merci pour ton soutien, ta patience et ta confiance. Ton calme m'apaise, tes conseils me rassurent, je suis tellement fière d'avancer à tes côtés. Notre amour a toujours été pour toi une évidence. Merci de rendre ma vie chaque jour plus belle. Nos premières années ensemble furent magnifiques et je le sais, ne sont qu'un préambule de tout ce qui nous reste à vivre. Je t'aime.

A mon frère Hugo, ma fierté, merci pour ta joie de vivre, merci pour ton amour. A notre enfance, nos joies, nos peines, notre complicité, à toutes ces choses qui nous ont construit ensemble et qui ont tissé entre nous un lien indéfectible. Je t'aime.

A Claudia, merci de faire de mon frère un homme heureux et épanoui, tu es un vrai rayon de soleil dans notre famille.

A ma grand-mère Angèle, merci pour ton immense soutien tout au long de mes études. Depuis notre naissance à Hugo et moi, tu nous as placés au centre de tout et ces quelques mots ne suffisent pas à te remercier pour tout ce que tu m'as apportée.

A ma grand-mère Enza, parce qu'une mamie c'est comme une maman! Merci pour toute la tendresse et l'amour que tu me donnes depuis ma plus tendre enfance.

A ma belle-famille, Blandine, Patrick et Olivier. Merci de m'avoir accueilli avec tant de générosité dans votre famille. Merci pour votre soutien et votre gentillesse, j'ai de la chance de vous avoir et je suis heureuse de devenir très bientôt une Kuffler pour de bon!

A mes oncles, tantes, cousins et cousines : Fanfan, Jean Jean, Nadège, Léo, Marjorie, Nicolas et Romain.

\section{A mes amis :}

A Marine, avec toi c'est l'amitié avec un grand A. Merci d'avoir traversé toutes ces années à mes côtés, merci pour ta gentillesse, ta générosité et ton amour. Je te l'ai déjà dit, tu es la sœur que je n'ai pas eue. Je t'admire pour la femme et le médecin que tu es devenue et je suis fière d'être ton amie. J'ai des souvenirs magnifiques avec toi et j'espère que le futur nous réserve encore plein de beaux moments ensemble. 
A Marie, ma cantoch. Ton départ à Paris a été un déchirement, mais heureusement j'ai trouvé le moyen de t'y rejoindre de temps en temps... Merci pour ta joie de vivre, ta gentillesse, ton intelligence. Je ne connais personne qui ait autant le sens de l'amitié que toi.

A Laura, ma mussmuss. Ces années médecine n'auraient pas été les mêmes sans toi. Dès que je t'ai rencontrée ça a été le coup de cœur. Merci pour notre complicité, pour notre amitié qui traverse les années.

A Romain, merci d'être tout simplement toi, tu es un ami en or.

A toute ma joyeuse troupe, vous êtes devenus des amis, des vrais! A Julie ma beauté, à Alex ma star («ma star? C'est-à-dire ?»), à Céline la plus brillante de tous les urgentistes!, à Adeline pour ta joie de vivre, à Sonia la plus marrante de toutes, à Ariane la casse-cou en roller derby, sans oublier les garçons : à Raph pour ta bonne humeur légendaire, à Jb le sexy-pompier par excellence, à Vincent notre petit bébé et à Marco le chirurgien syndicaliste de la troupe! Je suis une troupe, tu es une troupe, nous sommes une troupe; je vous aime, tendresse et chocolat.

A Elodie ma copine de toujours. Je vous souhaite avec John beaucoup de bonheur. Merci à tous les deux de faire partie de nos vies.

A Coralie ma copine depuis les bancs de la maternelle. Merci ma Coco, notre amitié est si chère à mes yeux. Merci à Denise d'avoir accepté de devenir ami avec mes parents! C'était notre rêve avec Coco et on a réussi ! Notre voyage tous ensemble en Thaïlande reste un souvenir inoubliable. Merci à Baptiste de rendre heureuse ma copine et à bébé Oscar de lui permettre de s'épanouir en tant que maman!

A Laura, ma pipich, merci pour ces années lycées qui furent sans doute les plus belles ; pour notre insouciance, notre énergie, notre complicité, nos folles soirées de concert et nos folles journées de glisse. Merci de toujours être à mes côtés.

A Sarah, que de beaux souvenirs ensemble, nos deux mois au Vietnam sont inoubliables. Merci pour ton amitié et ta gentillesse ma Sari.

A Marie-Gabrielle, que de fou rire avec toi pendant ces années de fac ! Merci à Guigui de te rendre heureuse, l'avenir vous réserve de belles surprises, vivement l'été prochain!

Aux copains de promo : Jean, Claire, TomTom, Matthias, Lory, Thomas, Charles et Romano (sans le VVV ces années n'auraient pas été les mêmes !), Clémentine, Maeva et tous les autres...

A mes amis de la Colle : Adrien, Florian, Marine et Lina, Eléna, Elise, Thomas, Lulu et Nicole, Nathalie et Jean-Luc, Mumu et Pierre, Josette, Christine, Nico, Richard et Yohan. 
A Caro, Juju et Antoine, Jean-Mi, Angelica, Anto, Julie ma juju, Florence la floump, Seffat, Crock et Popo ; merci d'être à nos côtés et de faire partie de nos vies.

A ceux qui ne sont plus là mais qui ont tant compté :

A mes deux grands-pères mon pépé Gaby et mon pépé Jo, bien plus que des grandspères je sais que vous êtes fiers de moi de là où vous êtes et que vous veillez sur moi, mes anges gardiens.

A mémé Olga et pépé Marius, mémé d'Italie et mamie Paulette,

A nos amis partis trop tôt, Luc et Jean vous êtes dans mon cœur. 


\section{TABLE DES MATIÈRES}

ABRÉVIATIONS .......................................................................................................................20

INTRODUCTION ...................................................................................................21

MISE AU POINT : SYNDROMES PARKINSONIENS, SYSTÈME DOPAMINERGIQUE ET TEP

CÉRÉBRALE A LA 18F-FDOPA

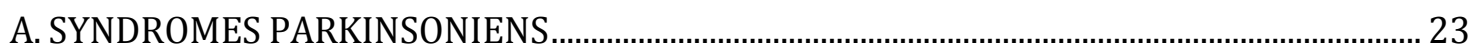

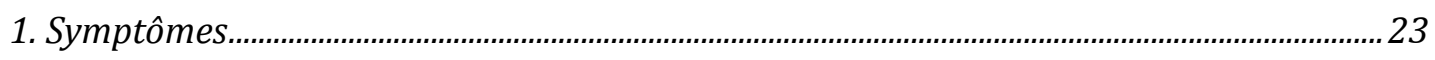

2. Etiologies des syndromes parkinsoniens .................................................................................... 24

3. Epidémiologie de la maladie de Parkinson ……........................................................................24

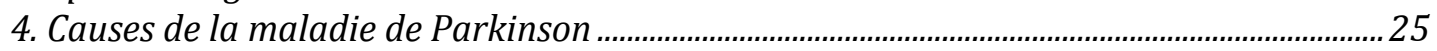

B. PHYSIOPATHOLOGIE : LA DOPAMINE

C. BIODISTRIBUTION ET PHARMACOCINÉTIQUE DE LA 18 F-FDOPA....................................... 29

D. INTERPRÉTATION VISUELLE ET SEMI-QUANTITATIVE DE LA TEP CÉRÉBRALE A LA

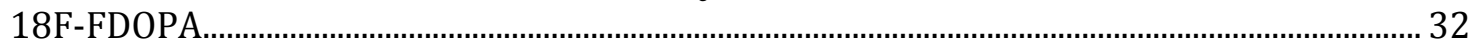

1. Critères visuels d'interprétation .................................................................................................. 32

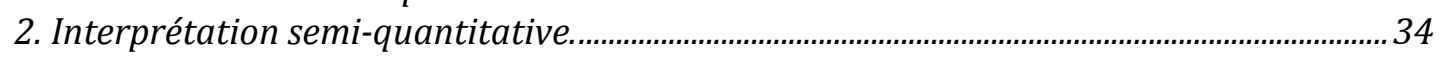

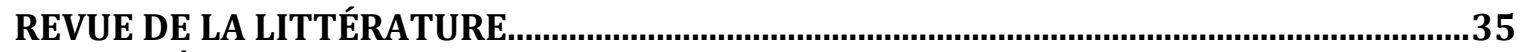

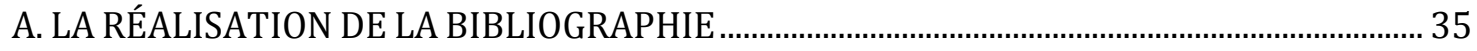

B. PERFORMANCES DE LA TEP A LA 18F-FDOPA DANS LE DIAGNOSTIC POSITIF DE LA

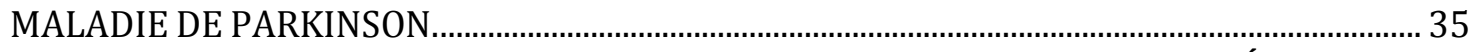

C. PERFORMANCES DE LA TEP A LA 18F-FDOPA DANS LE DIAGNOSTIC DIFFÉRENTIEL

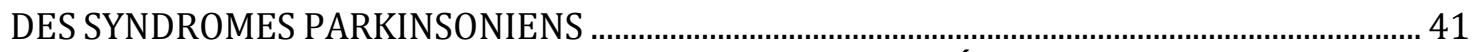

D. CONCLUSION ET DISCUSSION DE LA REVUE DE LA LITTÉRATURE ..................................... 45

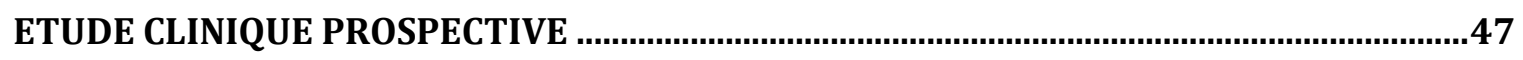

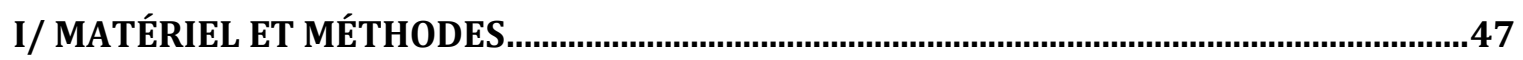

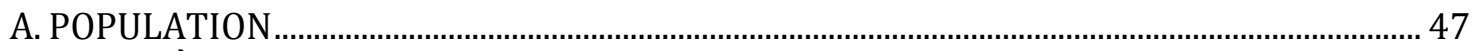

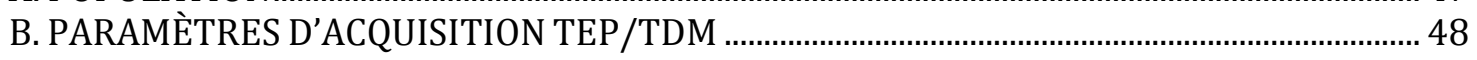

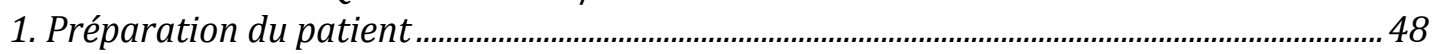

2. Réalisation de l'examen .................................................................................................................. 48

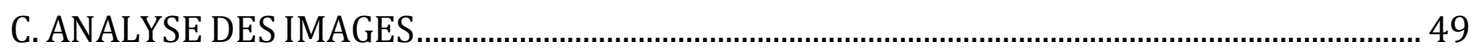

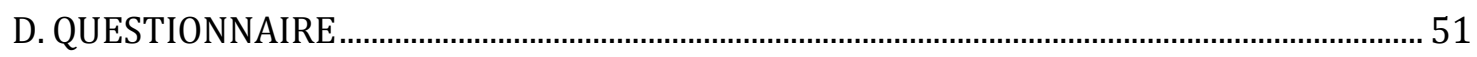

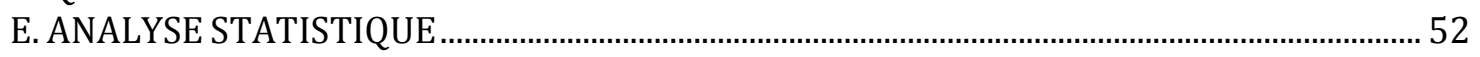

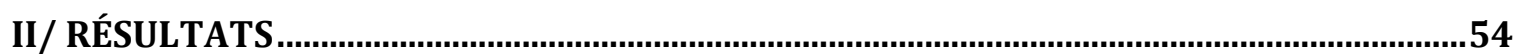

A. RÉSULTATS DE L'ÉVALUATION CLINIQUE ....................................................................... 54

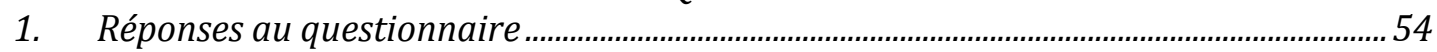

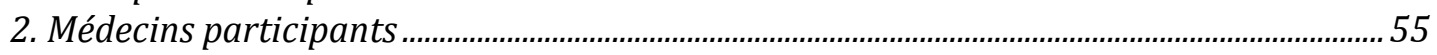

3. Caractéristiques de la population des patients .......................................................................56

4. Evaluation du cheminement diagnostique avant et après la TEP........................................5 57

5. Evaluation de l'intérêt de la TEP cérébrale à la 18F-FDOPA par les neurologues............ 64

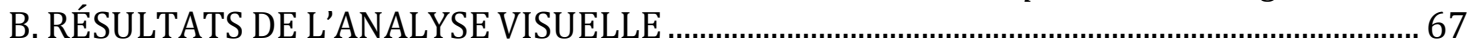

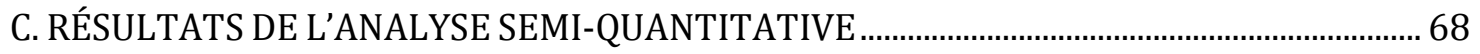

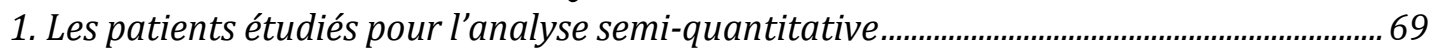

2. Etude de reproductibilité inter-observateur....................................................................... 70

3. Etudes préliminaires à l'analyse semi-quantitative ..................................................................

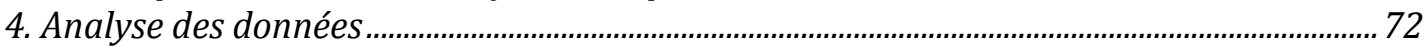

4.1 Valeurs semi-quantitatives...........................................................................................................................................72

4.2 Courbes ROC : figures de mérite SUV controlatéraux et homolatéraux à l'atteinte motrice

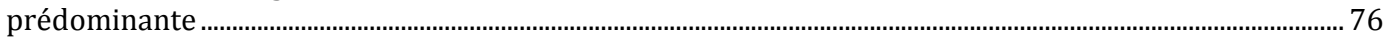

4.3 Courbes ROC : figures de mérite SOR, COR et POR controlatéraux et homolatéraux à l'atteinte motrice prédominante. 
III/ DISCUSSION

CONCLUSION

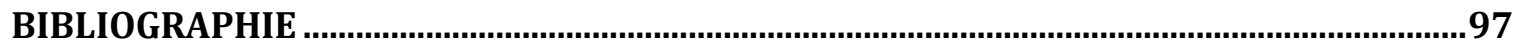

A. ANNEXE 1 : QUESTIONNAIRE ENVOYE AUX MEDECINS NEUROLOGUES PRESCRIPTEURS ...................101

B. ANNEXE 2 : FICHE D'INTERPRETATION DU MEDECIN NUCLEAIRE ......................................................102

TABLE DES ILLUSTRATIONS

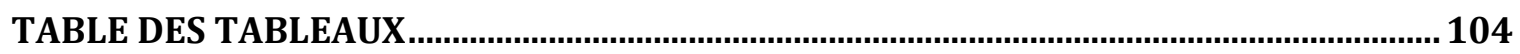

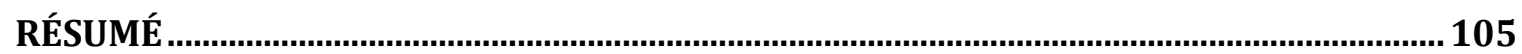

ABSTRACT

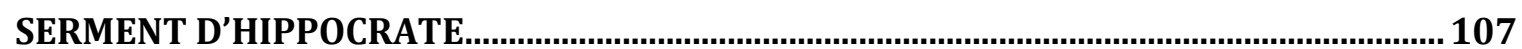




\section{ABRÉVIATIONS}

AADC : aromatic acid decarboxylase

AMM : autorisation de mise sur le marché

AMS : atrophie multi-systématisée

ATV : aire tegmentale ventrale

BHE : barrière hémato-encéphalique

CHU : centre hospitalier universitaire

COMT : catécholamine-0-méthyltransférase

COR : caudé occipital ratio

DAT : dopamine active transporteur

DCB : dégénérescence cortico-basale

DCL : démence à corps de lewy

DDC : dopa-décarboxylase

DFT : démence fronto-temporale

HPN : hydrocéphalie à pression normale

INCER : institut des neurosciences cliniques de Rennes

INSERM : institut national de la santé et de la recherche médicale

LAT1 : large neutral amino acid transporter type 1

L-DOPA : L-dihydroxyphénylalaline

MP : maladie de parkinson

POR : putamen occipital ratio

PSP : paralysie supra-nucléaire progressive

ROC : receiving operating characteristics

SOR : striatal occipital ratio

SUV : standardized uptake value

TDM : tomodensitométrie

TEMP : tomographie à émission mono-photonique

TEP : tomographie à émission de positon

VMAT2 : vesicular monoamine transporter type 2

VOI : volume of interest 


\section{INTRODUCTION}

Les pathologies neurodégénératives sont un problème de santé publique majeur dans les pays industrialisés dont l'âge moyen de la population est en constante augmentation. La maladie de Parkinson (MP) est la seconde maladie neurodégénérative la plus fréquente après la maladie d'Alzheimer, elle est caractérisée par la disparition prématurée, lente et progressive des neurones dopaminergiques au niveau des noyaux gris centraux, en particulier de la substance noire pars compacta, conduisant à un déficit en dopamine dans les zones de projection et en particulier le striatum.

Le syndrome parkinsonien, aussi appelé syndrome extra-pyramidal est composé de la triade clinique tremblements, rigidité et akinésie. Dans certaines situations, devant la présence d'une triade incomplète, de symptômes peu marqués, devant la suspicion d'un syndrome parkinsonien secondaire ou encore l'absence de réponse thérapeutique, le clinicien peut être amené à prescrire un examen complémentaire. La tomographie d'émission monophotonique (TEMP) utilisant le 123I-FP-CIT (DaTSCAN®) est encore considérée comme «la méthode d'imagerie de référence »(1,2) dans le diagnostic des syndromes parkinsoniens. En effet, chaque service de médecine nucléaire dispose d'une caméra TEMP nécessaire à la réalisation de l'examen et le radiopharmaceutique, bien que couteux, est facilement accessible car disponible depuis longtemps. Pourtant avec l'arrivée des caméras TEP TDM dans les services de médecine nucléaire et la distribution commerciale de la 18F-FDOPA, il est désormais possible de réaliser la TEP cérébrale à la 18F-FDOPA en routine clinique, nous offrant une meilleure sensibilité et amélioration de la résolution spatiale.

Au sein du service de médecine nucléaire du Centre Antoine Lacassagne, nous avons fait le choix d'abandonner le DaTSCAN® et de réaliser une TEP cérébrale à la 18F-FDOPA. D'une part ce dernier examen nous permet de proposer à nos correspondants des images de meilleure qualité en augmentant la sensibilité et la résolution spatiale et d'autre part il permet un gain de temps non négligeable en diminuant le temps de présence du patient dans le service. En effet, les délais 
injection - acquisition ainsi que le temps d'acquisition sont considérablement réduits, y compris en administrant la Carbidopa une heure avant l'injection du radiopharmaceutique. Le patient reste dans notre service environ 2 heures et demi à 3 heures contre 4 à 6 heures pour un DaTSCAN®. De plus en pratique, le radiotraceur peut être utilisé pour des indications oncologiques comme le bilan d'extension ou de suivi des tumeurs neuro-endocrines digestives, les insulinomes ou encore les cancers médullaires de la thyroïde, des tumeurs cérébrales, des phéochromocytomes et para-gangliomes.

Notre travail a consisté dans un premier temps à réaliser une revue de la littérature afin de vérifier que la TEP cérébrale à la 18F-FDOPA, est un examen fiable et robuste pour le diagnostic de la maladie de Parkinson et ce malgré les arguments des détracteurs qui pensent que contrairement au DaTSCAN ${ }$, la TEP cérébrale serait moins sensible au stade précoce de la maladie en raison de mécanismes qui compensent la perte dopaminergique par une augmentation de l'activité de la dopa décarboxylase.

Dans un second temps, ce changement d'imagerie en pratique clinique nous a conduits à réaliser une étude clinique prospective monocentrique afin d'évaluer auprès de nos médecins neurologues prescripteurs l'utilité clinique de la TEP cérébrale à la 18F-FDOPA dans la prise en charge des syndromes parkinsoniens. Enfin, nous avons réalisé une analyse semi-quantitative des images afin de déterminer quels seuils de fixation permettent de différencier les patients atteints des patients sains. 


\section{MISE AU POINT : SYNDROMES PARKINSONIENS, SYSTÈME DOPAMINERGIQUE ET TEP CÉRÉBRALE A LA 18F-FDOPA}

\section{A. SYNDROMES PARKINSONIENS}

\section{Symptômes}

Les syndromes parkinsoniens ou extra-pyramidaux dont fait partie la maladie de Parkinson (MP), regroupent les maladies présentant la triade clinique de symptômes moteurs: tremblement, akinésie et rigidité. Le tremblement parkinsonien est un tremblement de repos qui disparaît lors des mouvements volontaires ou lors du maintien d'une attitude. Il peut être augmenté par l'émotion ou la concentration. Il peut siéger aux membres supérieurs ou inférieurs, aux lèvres ou au menton mais pas à l'ensemble de la tête et peut être unilatéral. L'akinésie est caractérisée par la réduction et la lenteur des gestes, avec des mouvements plus rares, lents et parfois impossibles. Elle se manifeste par la marche à petits pas, et un faciès figé (perte des mouvements automatiques comme le clignement oculaire). Enfin la rigidité aussi appelée hypertonie extra-pyramidale s'objective par la résistance aux mouvements passifs imposés aux membres par l'examinateur (phénomène de la roue dentée). Cette triade symptomatique peut aussi atteindre la parole avec la dysarthrie parkinsonienne (élocution monotone, troubles du débit) ainsi que l'écriture avec la micrographie.

Selon les patients, certains symptômes peuvent être plus ou moins marqués donnant autant de formes cliniques.

Par ailleurs, le clinicien peut également retrouver des symptômes non moteurs tels que la douleur, des troubles psychiques comme la dépression, des troubles cognitifs, des troubles du sommeil, des troubles de l'équilibre, la perte de l'odorat ou encore une dysautonomie. Ces symptômes résultent probablement des répercussions de la maladie sur des structures cérébrales non dopaminergiques. 


\section{Etiologies des syndromes parkinsoniens}

La première étiologie est la maladie de Parkinson idiopathique. Ensuite on retrouve les syndromes parkinsoniens dits secondaires :

- la démence à corps de Lewy (DCL) : triade extra-pyramidale, troubles sphinctériens, hallucinations visuelles et des troubles cognitifs fluctuants ;

- la paralysie supra-nucléaire progressive ou maladie de Steele-RichardsonOlszewski (PSP): syndrome akinéto-rigide, troubles cognitifs frontaux, troubles de l'équilibre et de la marche, dystonie, dysarthrie et dysphagie ;

- l'atrophie multi-systématisée (AMS) : dysautonomie (hypotension orthostatique, troubles génito-sphinctériens), syndromes cérébelleux et pyramidal peuvent être associés ;

- la dégénérescence cortico-basale (DCB): triade extra-pyramidale et atrophie fronto-pariétale ;

- d'origine iatrogène, dus en général aux neuroleptiques (mais d'autres médicaments peuvent en être responsables) avec un syndrome extrapyramidal plutôt symétrique ;

- d'origine vasculaire: secondaire à des accidents ischémiques cérébraux malformation artério-veineuse. (3)

- l'hydrocéphalie à pression normale

- d'origine post-traumatique : traumatismes crâniens uniques ou répétés des boxeurs.

- d'origine toxique : maladie de Wilson, anoxie/hypoxie, hémochromatose.

\section{Epidémiologie de la maladie de Parkinson}

Selon l'INSERM (4) la maladie de Parkinson est au second rang des maladies neuro-dégénératives après la maladie d'Alzheimer. Elle est caractérisée par la destruction spécifique des neurones dopaminergiques de la substance noire du cerveau. Cette pathologie peut toucher aussi bien les hommes que les femmes. Selon le guide de l'HAS paru en juin 2014, un rassemblement des études de la prévalence et de l'incidence de la MP a été réalisé en 2005. La prévalence moyenne de la MP en 
France a ainsi été estimée à 827,5 pour 100000 habitants et environ 8000 nouveaux cas se déclarent par an dans notre pays. La MP touche les sujets âgés avec un pic autour de 70 ans, 1\% des plus de 65 ans sont concernés. Elle survient cependant dans 15\% des cas avant 40 ans. Compte-tenu de l'allongement de la durée de vie, l'incidence de la maladie progresse. La MP est de plus la seconde cause de handicap moteur d'origine neurologique chez le sujet âgé après les accidents vasculaires cérébraux.

\section{Causes de la maladie de Parkinson}

Les causes exactes demeurent à ce jour mal connues mais semblent être multifactorielles combinant des facteurs génétiques et environnementaux (5). En effet, il existe une susceptibilité génique à la maladie de Parkinson mais qui reste relativement faible. Selon l'INSERM, 21 variants génétiques associés à la maladie ont été identifiés en étudiant le génome de grandes cohortes de patients. Cependant, aucun de ces variants n'a d'effet suffisamment fort pour être prédictif de la maladie à un niveau individuel. Par exemple un sujet qui présente le profil génétique le plus défavorable voit son risque de développer la maladie multiplié par un facteur seulement égal à 2,5. La maladie de Parkinson n'est donc généralement pas une maladie héréditaire. Cependant, il existe environ $5 \%$ de formes génétiques, liées à des mutations affectant des gènes spécifiques (le gène de l' $\alpha$-synucléine, le gène LRRK-2 (leucine-rich repeat kinase 2) et le gène de la glucocerebrosidase (GBA)). Mais ces mutations ne sont pas systématiquement associées au développement de la maladie. A l'inverse le risque de développer la MP due à l'exposition aux pesticides a lui été établi, des études (6) ont montré l'existence d'un risque accru de la maladie de Parkinson chez les agriculteurs exposés aux insecticides de type organochlorés et la MP fait dorénavant partie du tableau des maladies professionnelles du régime agricole. D'autres facteurs environnementaux peuvent être protecteurs comme le tabac ou le café probablement par effet stimulant sur les neurones dopaminergiques. 


\section{B. PHYSIOPATHOLOGIE : LA DOPAMINE}

La dopamine est un neurotransmetteur appartenant aux catécholamines, elle est issue de la tyrosine. Son précurseur, la L-dihydroxyphénylalaline (L DOPA) qui est un acide aminé peut traverser la barrière hémato-encéphalique (BHE) contrairement à la dopamine (figure 1).

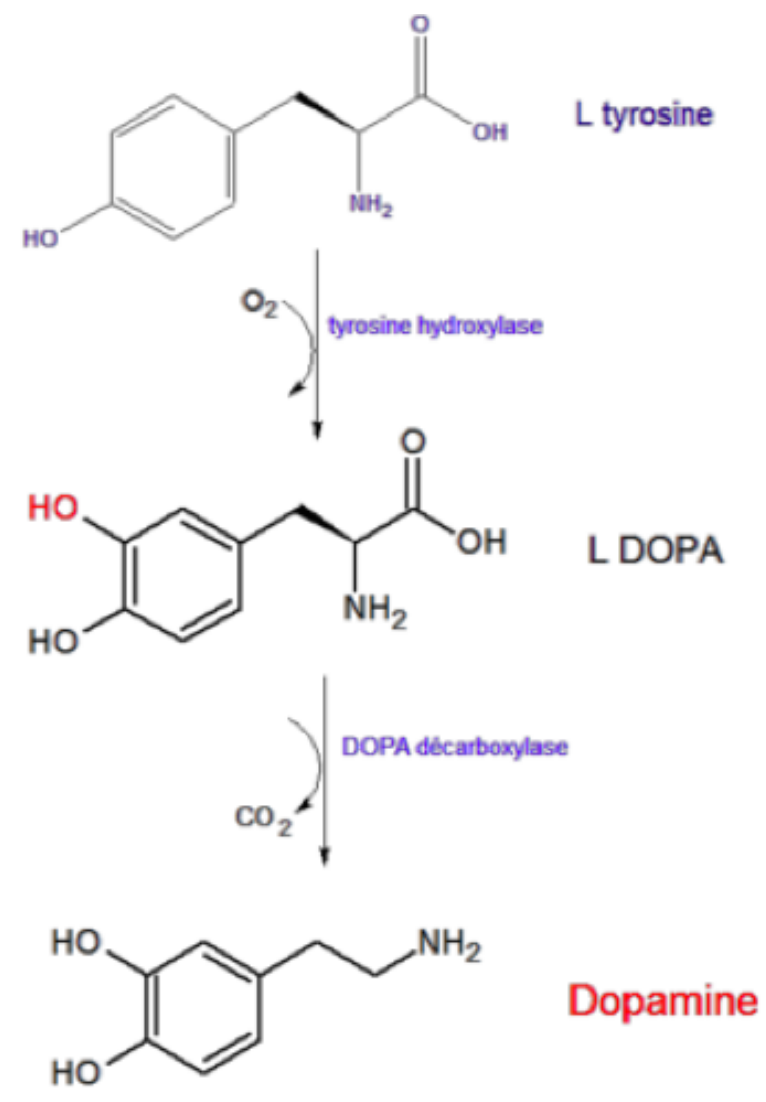

Figure 1 : L tyrosine, L DOPA et Dopamine

La dopamine est produite dans le mésencéphale principalement au niveau de la substance noire et de l'aire tegmentale ventrale (ATV) (figure 2). 

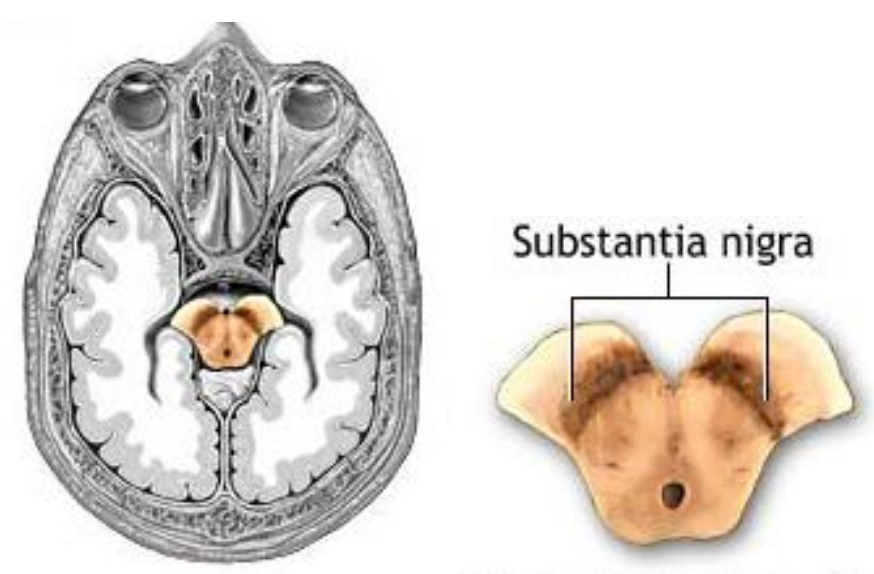

Figure 2 : Aire tegmentale ventrale

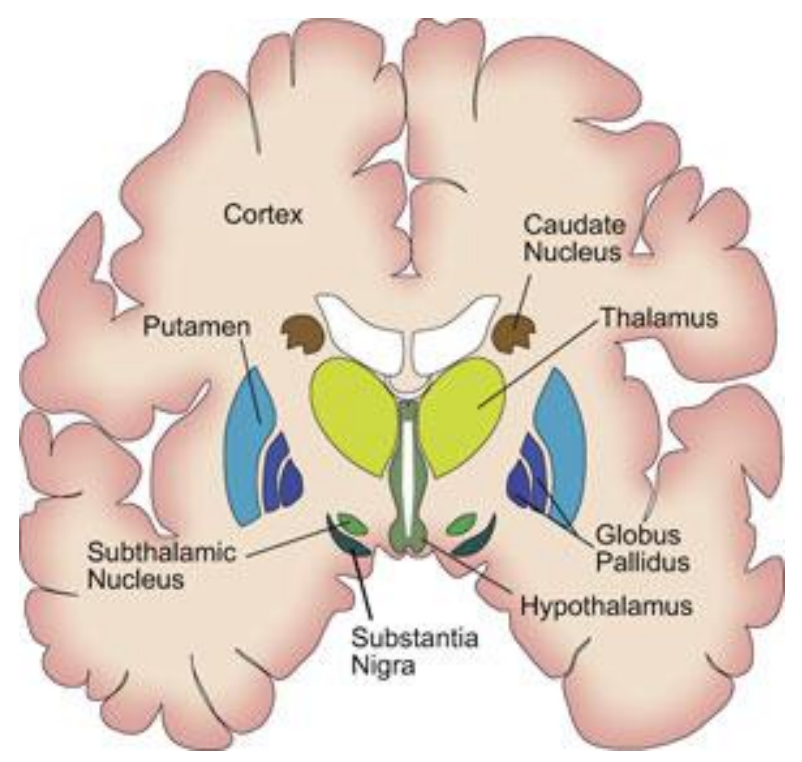

Figure 3 : Schéma du cerveau en coupe coronale et des noyaux gris centraux

La dopamine a un rôle de modulateur final des fonctions motrices et psychiques et elle intervient dans trois voies différentes :

- La voie nigro-striée : substance noire, noyau caudé et putamen (qui forment le striatum) (cf figure 3: schéma des noyaux gris centraux). Cette voie constitue le système modulateur des aires corticales motrices et intervient dans le contrôle des fonctions motrices.

- La voie méso-limbique: ATV, noyau accumbens, septum, amygdale, hippocampe. Ce système régule la motivation et la récompense (impliqué également dans l'addiction). 
- La voie méso-corticale: ATV, cortex fronto-cingulaire et entorhinal. Ce système est impliqué dans la concentration et les fonctions exécutives (mémoire de travail).

En plus d'être un neurotransmetteur, la dopamine est aussi une neurohormone, produite dans l'hypothalamus (figure 3). Sa principale fonction hormonale est d'inhiber la libération de prolactine au niveau de l'hypophyse antérieure.

Dans la maladie de Parkinson, il existe une dégénérescence sélective des neurones dopaminergiques du mésencéphale en particulier des neurones constituant la substance noire (voie nigro-striée). Les neurones dopaminergiques sont riches en neuro-mélanine ce qui leur confère une pigmentation noire visible sur coupe (figure 4). La dénervation dopaminergique induit une dépigmentation de cette zone chez les sujets MP. On observe également des inclusions cytoplasmiques éosinophiles caractéristiques au sein du corps de ces neurones : les corps de Lewy (figure 5). Ces neurones ayant des prolongements axonaux vers le striatum, la neurotransmission se trouve affectée d'abord au niveau des putamens puis des noyaux caudés. Bien que le diagnostic de certitude de la MP soit post-mortem, on comprend tout l'intérêt de l'imagerie fonctionnelle de la neurotransmission dopaminergique et en particulier de la TEP cérébrale à la 18F-FDOPA dans l'aide au diagnostic de cette pathologie. 


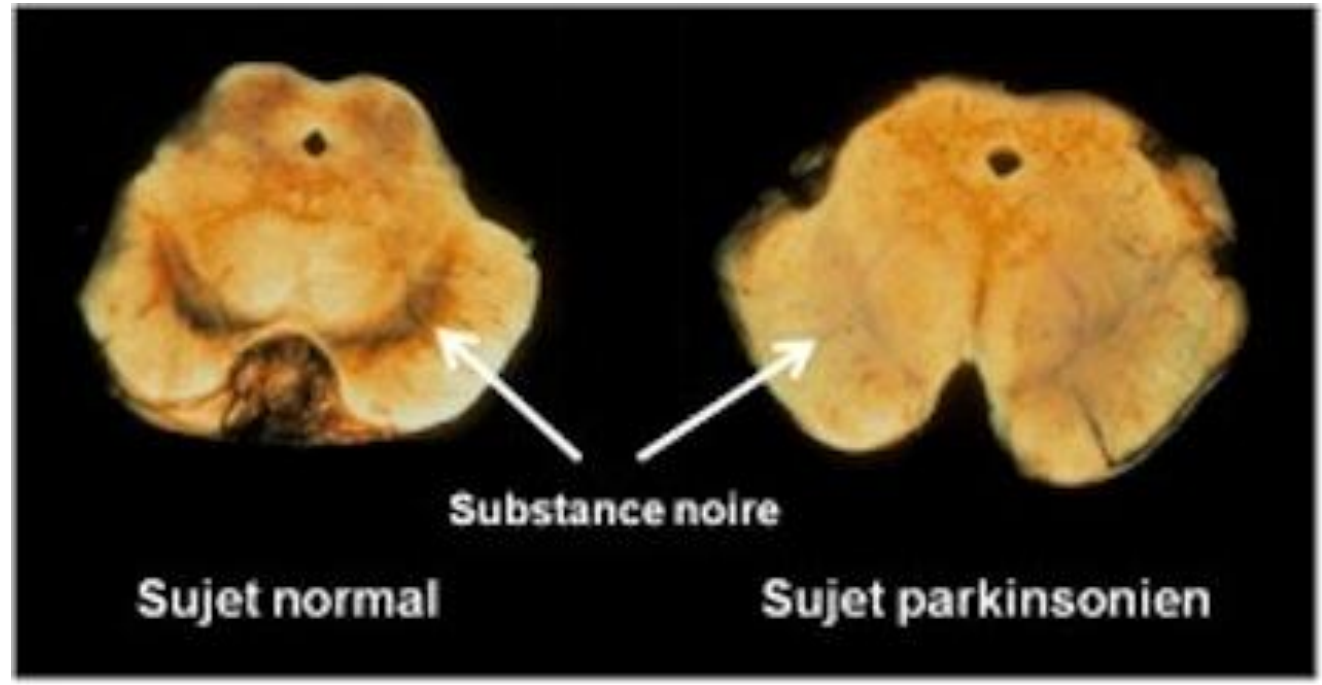

Figure 4 : Coupes de mésencéphale humain humain illustrant la dépigmentation de la substance noire chez un sujet parkinsonien (Images de l'INCR : institut des neurosciences cliniques de Rennes)

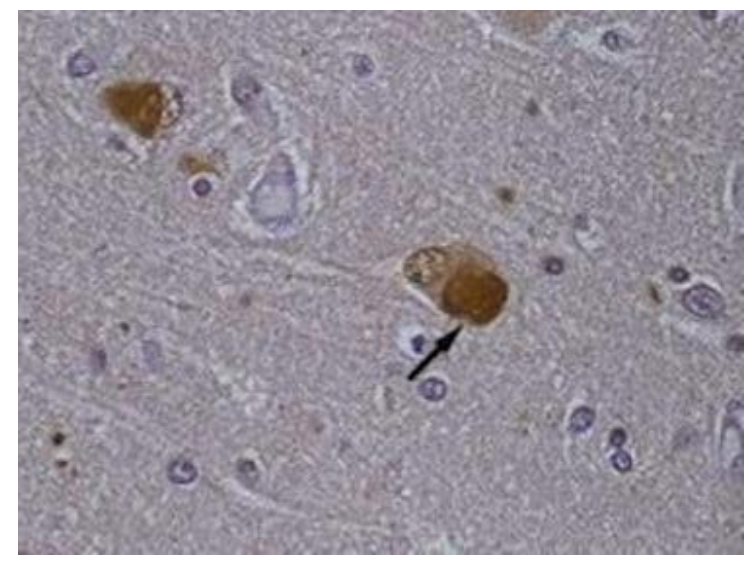

Figure 5 : Marquage par un anticorps anti-ubiquitine révélant la présence de corps de Lewy au sein des neurones de la substance noire.

\section{BIODISTRIBUTION ET PHARMACOCINÉTIQUE DE LA 18 F-FDOPA}

La 18F-FDOPA est un radiopharmaceutique composé de Ldihydroxyphénylalaline (L-DOPA) marqué au fluor 18. Après l'administration intraveineuse de la 18F-FDOPA, le radiopharmaceutique a la capacité de traverser la BHE grâce au «Large neutral Amino acid Transporter type 1 » (LAT1 : SLC7A5), il est ensuite converti en fluoro-dopamine dans les neurones dopaminergiques grâce à l'activité enzymatique de la dopa-décarboxylase (DDC) (Figure 6). A la différence de la L-DOPA et de son précurseur la tyrosine qui sont des acides aminés, la dopamine 
n'en est pas un. Ce radiopharmaceutique est indirectement le reflet du nombre de neurones dopaminergiques via l'activité enzymatique de la dopa-décarboxylase au sein de ces neurones.

Le radiomarqueur fluoré suit ensuite le même chemin que la dopamine, il est chargé dans les vésicules synaptiques par le transporteur VMAT-2, libéré dans la fente synaptique par exocytose, en partie capté par les récepteurs post-synaptiques, recapté par les neurones dopaminergiques pré-synaptiques par le DAT (dopamine active transporteur) puis dégradé dans la fente synaptique par une ectoenzyme (la COMT = catécholamine-0-méthyltransférase) ou dans les mitochondries du neurone pré-synaptique par les monoamine-oxydases respectivement en acide homovanillique et en acide dihydroxyphénylacétique. Ces métabolites peuvent ensuite traverser la BHE, passer dans le plasma puis être éliminés par les reins.

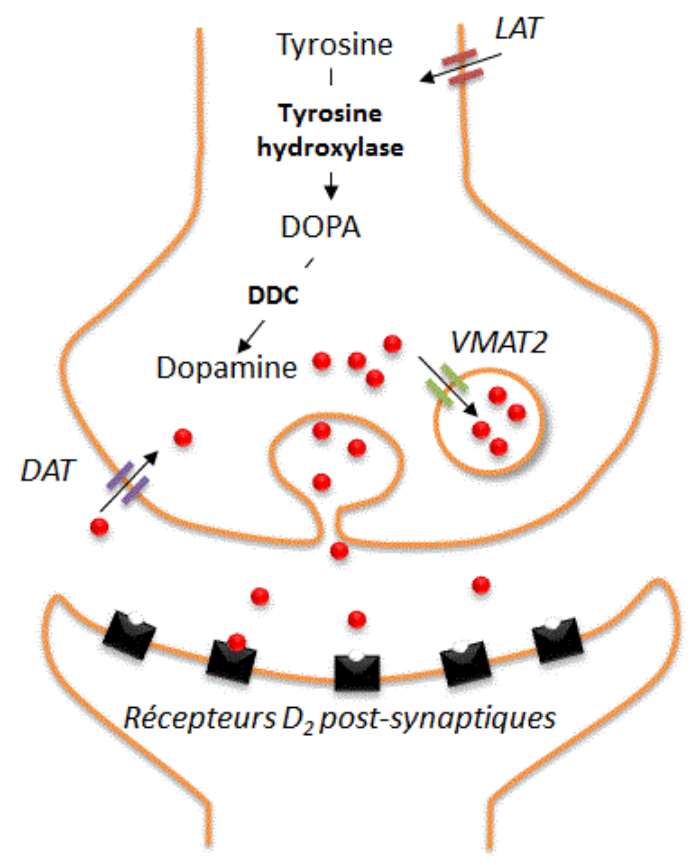

Figure 6 : Représentation schématique d'une synapse dopaminergique : LAT= large aminoacid transporter, DDC = dopa-décarboxylase, VMAT2 = type 2 vesicular monoamine transporter, DAT = dopamine transporter. Schéma adapté de (7) avec permission.

Pour mémoire, le 123I-FP-CIT (123I-fluoropropyl-2béta-carbomethoxy-3béta (4idodophényl) nortropane) connu sous le nom commercial de DatSCAN® est un radiopharmaceutique substrat transporteur de la dopamine (DAT). 
La fixation striatale du radiopharmaceutique atteint un plateau 30 à 45 minutes après l'administration puis décroît lentement à partir de 2 à 3 heures (8). La fixation extra-striatale du cerveau avoisinant atteint un maximum de fixation 30 minutes après injection mais décroît plus rapidement que la fixation striatale. En effet au sein des neurones dopaminergiques, le radiopharmaceutique va être métabolisé de la même façon que la dopamine, décarboxylé puis intégré dans les vésicules VMAT, libéré dans la fente synaptique et le plus souvent recapté par le DAT ce qui lui offre un temps de séjour beaucoup plus long qu'au sein des neurones non dopaminergiques avoisinants.

La fixation du radiotraceur au niveau cérébral représente seulement 0,2\% de l'activité administrée (9). C'est pour pallier ce faible pourcentage de fixation, qu'il est possible d'administrer au patient de la Carbidopa avant l'injection de 18FFDOPA, afin de bloquer l'activité enzymatique de la Dopadécarboxylase (DDC) périphérique en particulier au niveau digestif. L'étude de Hoffman a montré une augmentation des taux plasmatiques de 18F-FDOPA ainsi qu'une augmentation de l'activité spécifique (striatale) et non spécifique (cérébelleuse) chez les patients à qui on administrait par voie orale de la Carbidopa avant l'injection de 18F-FDOPA (10). Dans cette étude l'augmentation de la captation spécifique est de l'ordre de 1,5 après $100 \mathrm{mg}$ de carbidopa et de 2 après 250 mg de carbidopa ; l'augmentation de la captation non spécifique est respectivement augmentée de 1,2 et 1,4. L'étude de Brown (11) sur la dosimétrie de la 18F-FDOPA dans le corps entier, deux heures après l' administration de 100mg de Carbidopa, a montré une diminution de l'excrétion urinaire à $2 \mathrm{~h}$ et à $3 \mathrm{~h}$ après injection, un temps de résidence significativement augmenté du radiopharmaceutique au niveau du striatum par rapport à la fixation non spécifique et un bruit de fond cérébral équivalent à celui de l'abdomen. 


\section{INTERPRÉTATION VISUELLE ET SEMI-QUANTITATIVE DE LA TEP CÉRÉBRALE A}

\section{LA 18F-FDOPA}

\section{Critères visuels d'interprétation}

Visuellement on ne peut apprécier que la répartition relative de la fixation entre les diverses structures, et la quantification absolue reste très difficile voire impossible. D’après les données de la littérature, celles de notre propre expérience (12) ainsi que les données des études post-mortem la densité des neurones dopaminergiques chez les sujets sains est plus élevée dans le putamen que dans le noyau caudé et à l'inverse diminuée de façon plus marquée dans le putamen que dans le noyau caudé chez les patients MP (13). Nous avons donc retenu la diminution de fixation du putamen et la relative épargne du noyau caudé comme des critères majeurs de l'interprétation. Chez les sujets normaux, le maximum de fixation est situé dans le putamen antérieur ou postérieur (image 1) et chez les patients anormaux on observe une diminution de fixation commençant au niveau du putamen postérieur, puis antérieur avec un maximum de fixation situé dans le noyau caudé, l'atteinte est initialement asymétrique controlatérale aux symptômes dominants (image 2) puis avec l'évolution de la maladie elle devient bilatérale (image 3). Chez les patients très évolués, on retrouvera une fixation caudée quasiexclusive (image 4) avec une augmentation de la fixation cérébrale non spécifique autrement dit le bruit de fond cérébral. Nous recommandons d'utiliser une échelle de couleur qui permette de bien mettre en évidence le maximum de fixation, par exemple une échelle type « arc en ciel ». Afin que l'interprétation visuelle soit fiable, le maximum de fixation doit être situé dans le striatum. 
Figure 7 : Images de TEP cérébrale à la 18F-FDOPA en coupe axiale passant par les striatum réaxées dans le plan CA-CP.

Image 1 : image normale avec maximum de fixation dans le putamen antérieur ou postérieur

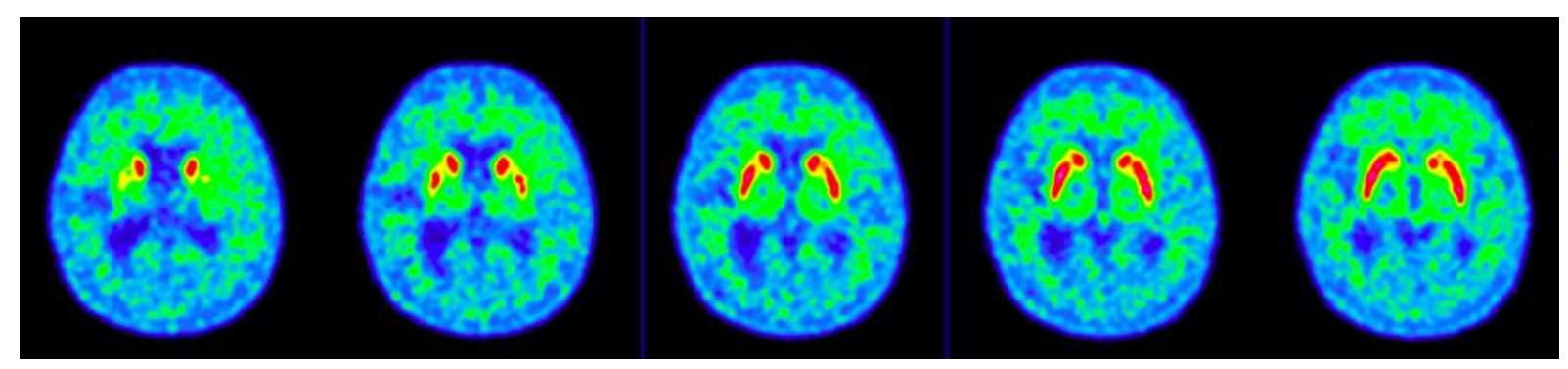

Image 2 : dénervation dopaminergique débutante avec hypofixation du putamen postérieur unilatérale

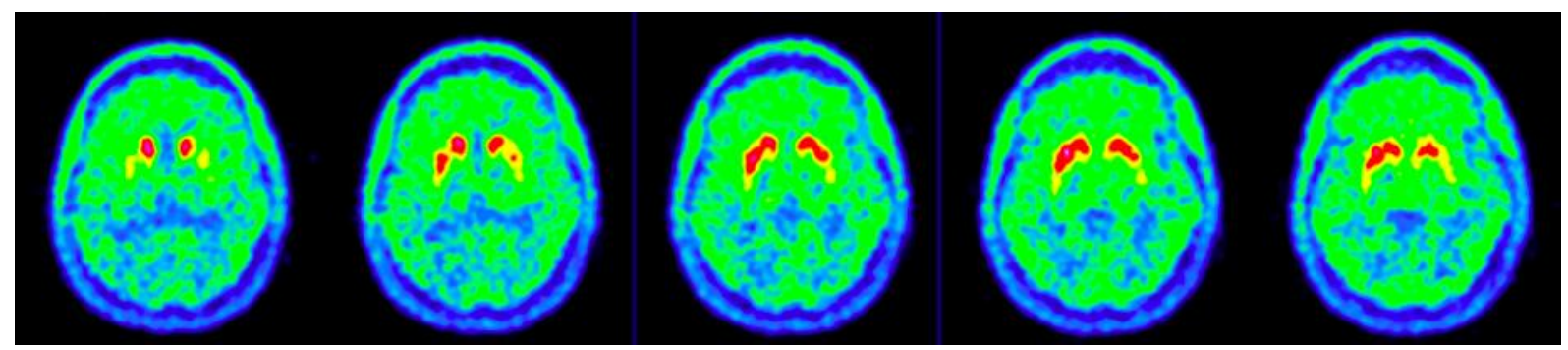

Image 3 : dénervation dopaminergique avec hypofixation putaminale bilatérale et maximium de fixation dans le noyau caudé

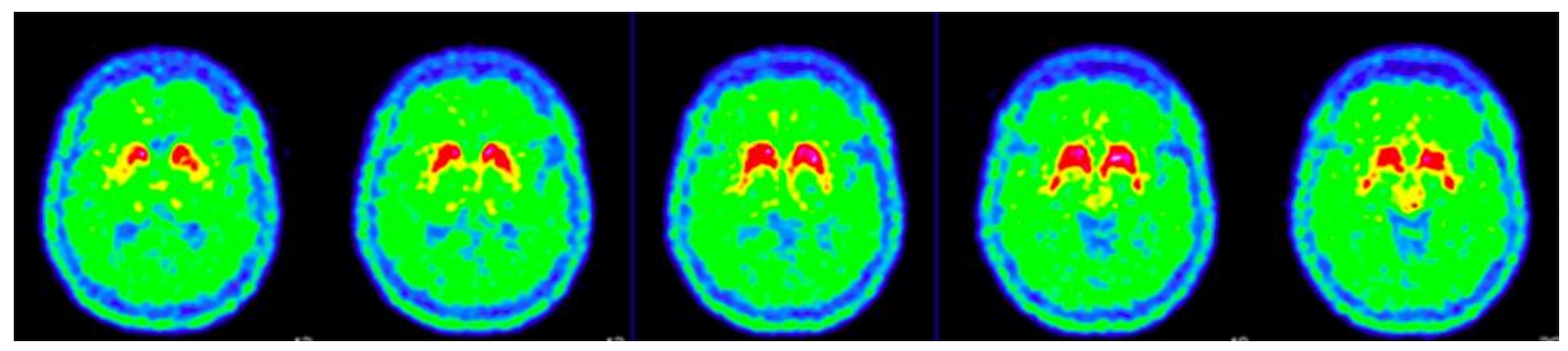

Image 4 : dénervation dopaminergique évoluée avec fixation caudée quasi-exclusive avec augmentation du bruit de fond cérébral

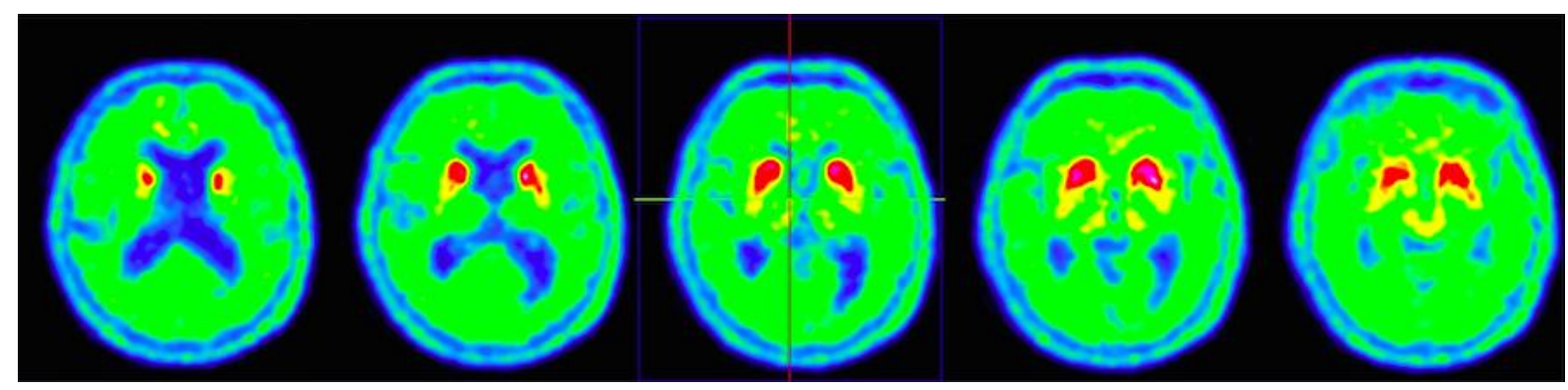




\section{Interprétation semi-quantitative.}

Pour plus de reproductibilité dans l'interprétation et afin que l'interprétation de la TEP cérébrale à la 18F-FDOPA ne soit pas opérateur dépendant, il est intéressant d'utiliser des mesures de fixation exploitées le plus souvent en termes de rapports: fixation spécifique (striatale, caudée ou putaminale antérieure ou postérieure) sur une fixation non spécifique (en général occipitale). Ils permettent aussi de s'affranchir des biais liés au fait de comparer les zones atteintes à un maximum de fixation qui est lui-même une zone hypofixante le plus souvent. Ces rapports de fixation sont appelés SOR en anglais (striatal to occipital ratio). Les premières études calculaient la constante d'influx Ki par analyse graphique, ce qui nécessitait de longues acquisitions dynamiques et des mesures plasmatiques fastidieuses réservées à la recherche mais il a été démontré que les SOR mesurés sur une image statique permettaient de différencier aussi bien les patients MP des patients sains que la constante Ki, permettant d'adapter l'analyse quantitative à la pratique clinique $(14,15)$. Afin de diminuer encore plus la variabilité interobservateur la tendance va au développement de logiciels d'analyse automatique avec des VOIs prédéfinies (16). Pourtant, à ce jour l'analyse visuelle reste la méthode de choix pour la pratique clinique de routine. 


\section{REVUE DE LA LITTÉRATURE}

La revue de la littérature des performances diagnostiques de la TEP cérébrale à la 18F-FDOPA dans les syndromes parkinsoniens a été un travail préliminaire à notre étude qui a conduit à une publication (7) en décembre 2014. La revue de la littérature a dans un premier temps porté sur le diagnostic positif de la maladie de Parkinson et dans un second temps sur les performances diagnostiques de la TEP cérébrale à la 18 F-FDOPA dans le diagnostic différentiel des syndromes parkinsoniens.

\section{A. LA RÉALISATION DE LA BIBLIOGRAPHIE}

La bibliographie a été effectuée avec le logiciel Zotero. Les documents ont été collectés grâce à des moteurs de recherche comme Pub Med, SUDOC, Google Scholar, et Google ainsi que les documents fournis par le Directeur de thèse.

Les mots clés utilisés étaient [positron-emission tomography], [18F-fluoro-L-dopa], [parkinson disease], [diagnosis], [parkinsonian syndrome], [extra-pyramidal syndrome].

\section{B. PERFORMANCES DE LA TEP A LA 18F-FDOPA DANS LE DIAGNOSTIC POSITIF DE}

\section{LA MALADIE DE PARKINSON.}

La revue de la littérature montre que de nombreuses études (Tableau 1) ont déjà cherché à évaluer les performances diagnostiques de la TEP cérébrale à la 18FFDOPA dans la maladie de parkinson aussi bien à des stades avancés qu'à des stades plus précoces.

On comprend ici toute l'importance de cet examen s'il permet de diagnostiquer la maladie de Parkinson à un stade précoce, tant en terme d'impact diagnostique, thérapeutique que pour le suivi du patient.

En 1990, D.J. Brooks décrivait déjà la place de l'imagerie fonctionnelle dans le diagnostic de la MP (17) ainsi que le modèle de fixation striatale typique de la 18F- 
FDOPA dans la maladie de Parkinson (18) avec une sévère hypofixation du putamen postérieur et une relative épargne du noyau caudé. Dans son étude, Rinne et al (19) a affiné ce modèle de fixation striatale en comparant les examens de patients MP précoces jamais traités à ceux de volontaires sains. L'hypofixation était la plus marquée dans le putamen postérieur (elle correspond à 28\% de la valeur de fixation chez les volontaires sains, $\mathrm{p}<0,0001$ ) par rapport au putamen antérieur. La fixation du noyau caudé était également réduite au niveau homo et controlatéral mais de façon moins marquée (69\% de la fixation des volontaires sains, $p<0,003)$. Cette étude a montré, sur un petit échantillon, la réduction significative de la fixation striatale du radiopharmaceutique pour les MP précoces, en commençant par le putamen postérieur, puis le putamen antérieur et de façon moins marquée et plus tardive le noyau caudé.

Alors que chez les patients sains, le maximum de fixation est clairement situé au niveau du putamen (antérieur voire même postérieur) la présence du maximum de fixation dans le noyau caudé est un signe fort de l'atteinte dopaminergique. Nurmi et al ont étudié la progression annuelle de la maladie au niveau de différentes sous-régions striatales et ont montré que l'atteinte maximale était observée au niveau du putamen postérieur (20). Des études comme celles de Bruke et al (21) et Hilker et al (22) en réussissant à suivre sur plusieurs années des patients MP avec des TEP cérébrales à la 18F-FDOPA ont montré que l'aggravation de l'atteinte dopaminergique n'était pas linéaire dans le temps mais suivait une courbe exponentielle négative. La perte dopaminergique est rapidement progressive au début de la maladie puis ralentit avec l'aggravation des symptômes. Au cours de la progression de la maladie, alors que la fixation striatale diminue, la fixation extrastriatale augmente et ce phénomène a été décrit dans l'étude de Pavese et al (23) comme un effet compensateur.

Jokinen et al (14) a montré que la valeur du SOR (striatal occipital ratio) comme la valeur du Ki (constante calculée par analyse graphique) permettent de séparer les patients MP des volontaires sains. Le putamen postérieur controlatéral au côté de l'atteinte motrice prédominante était la valeur qui avait la plus grande aire sous la courbe (courbe ROC) $(0,994$ for SOR et 0,998 for Ki).

Les capacités de cet examen à détecter les MP précoces ont été spécifiquement étudiées. Dans son étude Morrish et al (24) a comparé 27 MP 
précoces avec 16 patients normaux. Parmi les MP précoces, 11 patients avaient un hémi parkinson (HP) pur et le striatum controlatéral au côté non affecté était considéré comme représentant d'une atteinte pré-symptomatique. L'étude a montré une diminution significative de la fixation putaminale comparativement aux sujets sains aussi bien pour le côté en rapport avec les lésions pré-symptomatiques que pour le côté en rapport avec l'HP précoce. Concernant le côté symptomatique, 25/27 patients avaient des valeurs de Ki inférieures de 2 DS à la normale. Pour le côté présymptomatique, la fixation était la plupart du temps inférieure à celle des patients normaux avec seulement 2/11 patients avec une valeur inférieure à 2 DS. L'étude de Nagasawa et al (25) retrouvait une diminution significative de la fixation aussi bien du côté atteint que du côté pré-symptomatique en comparant 10 HP avec 10 patients contrôles. Rinne et al (19) a montré qu'aussi bien la TEP cérébrale à la 18FFDOPA qu'au 18F CFT (Fluor 18 marqué avec un DAT ligand) était capable de détecter les MP précoces. Les valeurs de fixation au niveau du putamen antérieur et postérieur étaient diminuées de 3 DS par rapport aux valeurs des sujets contrôles. D’autres études ont comparé directement le DaTSCAN® (123 I-FP-CIT TEMP TDM), à la TEP cérébrale à la 18F-FDOPA. Eshuis et al (26) a comparé les deux examens chez 13 MP précoces et 17 MP avancées. En évaluant les valeurs de fixation du noyau caudé ils ont obtenu une sensibilité de $77 \%$ et une spécificité de $71 \%$ pour le DaTSCAN® et de 76\% et de 85\% pour la 18F-FDOPA. Dans une seconde étude (27) ils ont différencié les MP précoces des MP avancées et ont comparé les données de 11 MP précoces, 17 MP avancés et 20 patients sains. Ils ont obtenu une sensibilité et une spécificité de $100 \%$ pour les deux techniques en analysant les valeurs de fixation du putamen controlatéral à la symptomatologie motrice prédominante.

Comme vu précédemment, la progression de la maladie a intéressé beaucoup d'auteurs qui ont comparé l'aspect de la fixation de la 18F-FDOPA au cours du temps chez des patients MP et des patients sains. A l'inverse des radiopharmaceutiques utilisant des ligands DAT, il n'y avait pas de diminution significative de la fixation de la 18F-FDOPA au cours du temps chez les patients sains (28) (20) alors qu'on retrouvait bien une diminution progressive chez les patients MP (29) 


\begin{tabular}{|c|c|c|c|}
\hline $\begin{array}{l}\text { Etudes (premier } \\
\text { auteur et } \\
\text { année de publication) }\end{array}$ & $\begin{array}{l}\text { Population et } \\
\text { diagnostic clinique } \\
\text { Maladie de parkinson } \\
\text { et patients contrôles }\end{array}$ & Intérêt de l'étude & Résultats principaux \\
\hline Jokinen \& Al (2009) & $\begin{array}{l}\text { MP précoce }(n=89) \text { et } \\
\text { volontaires sains } \\
(n=21)\end{array}$ & $\begin{array}{l}\text { Séparer les MP } \\
\text { précoces } \\
\text { volontaires sains en } \\
\text { utilisant les rapports } \\
\text { de fixation (SOR) des } \\
\text { sous-régions striatales } \\
\text { en tenant compte de } \\
\text { l'asymétrie des } \\
\text { symptomes moteurs. }\end{array}$ & $\begin{array}{l}\text { Le SOR du putamen postérieur contro- } \\
\text { latéral à l'atteinte motrice } \\
\text { prédominante était significativement } \\
\text { diminué dans le groupe MP précoce. } \\
\text { Les valeurs du SOR et du Ki ref du } \\
\text { putamen postérieur controlatréral ont } \\
\text { montré la plus grande aire sous la } \\
\text { courbe (ROC): } 0,994 \text { pour le SOR et } \\
0.998 \text { pour le Ki ref et permettent donc } \\
\text { une excellente séparation entre les MP } \\
\text { précoces et les volontaires sains. }\end{array}$ \\
\hline Rinne \& Al (2001) & $\begin{array}{l}\text { MP précoce }(=8) \text { et } \\
\text { volontaires sains }(n=5)\end{array}$ & $\begin{array}{l}\text { Comparer les } \\
\text { performances de deux } \\
\text { radiotraceurs en TEP } \\
\text { TDM (18 FDOPA et } 18 \\
\text { FCFT) pour la } \\
\text { détection de l'atteinte } \\
\text { dopaminergique pré- } \\
\text { synaptique dans les } \\
\text { MP précoces. }\end{array}$ & $\begin{array}{l}\text { Les deux radiotraceurs ont la capacité } \\
\text { de détecter l'atteinte pré-synaptique } \\
\text { dopaminergique des MP précoces. Ils } \\
\text { ont montré une diminution } \\
\text { significative au niveau du putamen } \\
\text { postérieur, puis antérieur suivi du } \\
\text { noyau caudé. L'hypofixation la plus } \\
\text { marquée était située au niveau du } \\
\text { putamen postérieur controlatéral à } \\
\text { l'atteinte motrice. }\end{array}$ \\
\hline Eshuis \& Al (2006) & $\begin{array}{l}\text { MP ( } n=30 \text { comprenant } \\
\text { des MP précoces } n=13 \\
\text { et avancées } n=17 \text { ) }\end{array}$ & $\begin{array}{l}\text { Comparaison des } \\
\text { performances de la } \\
\text { 18F FDOPA TEP TDM } \\
\text { et du DaTSCAN® chez } \\
\text { les mêmes patients, } \\
\text { MP précoce et } \\
\text { avancée. }\end{array}$ & $\begin{array}{l}\text { La sensibilité de la 18F-FDOPA TEP } \\
\text { TDM pour différencier les patients } \\
\text { entre les deux groupes était } 0,88 \text { et la } \\
\text { spécificté } 0,77 \text {. Pas de différence } \\
\text { significative entre TEP et DaTSCAN®. }\end{array}$ \\
\hline
\end{tabular}




\begin{tabular}{|c|c|c|c|}
\hline Eshuis \& Al (2009) & $\begin{array}{l}\text { MP précoce }(n=11) \text {, } \\
\text { MP avancée }(n=17) \text { et } \\
\text { volontaires sains } \\
(n=20)\end{array}$ & $\begin{array}{l}\text { Déterminer et } \\
\text { comparer la sensibilité } \\
\text { et la specificité de la } \\
\text { TEP DOPA et du } \\
\text { DaTSCAN@ dans le } \\
\text { diagnostic des déficits } \\
\text { dopaminergiques } \\
\text { striataux. }\end{array}$ & $\begin{array}{l}\text { La TEP DOPA et le DaTSCAN@ sont } \\
\text { tous deux capables de diagnostiquer } \\
\text { une atteinte dopaminergique } \\
\text { présynaptique y compris à un stade } \\
\text { précoce avec une excellente sensibilité } \\
\text { (91\% pour les deux techniques) et } \\
\text { spécificité (respectivement } 90 \text { et } 100 \\
\%) \text {. }\end{array}$ \\
\hline Nurmi \& Al (2001) & $\begin{array}{l}\text { Patients MP }(n=21) \text {, } \\
\text { volontaires } \\
(n=8) .\end{array}$ & $\begin{array}{l}\text { Evaluer la progression } \\
\text { de la maladie de } \\
\text { Parkinson grâce à la } \\
\text { TEP DOPA réalisée à } 5 \\
\text { ans d'intervalle. }\end{array}$ & $\begin{array}{l}\text { Chez les volontaires sains, pas de } \\
\text { diminution singificative de la fixation } \\
\text { de la DOPA. Ches les MP, l'atteinte } \\
\text { débute dans le putamen postérieur, } \\
\text { puis le putamen antérieur, et ensuite le } \\
\text { noyau caudé. }\end{array}$ \\
\hline Pavese \& Al (2011) & $\begin{array}{l}\text { Patients MP précoces } \\
(\mathrm{n}=10)\end{array}$ & $\begin{array}{l}\text { Evaluer la diminution } \\
\text { de la fixation de la F- } \\
\text { FDOPA dans les } \\
\text { structures cérébrales } \\
\text { noradrénergiques, } \\
\text { serotoninergiques et } \\
\text { extrastriatales } \\
\text { pendant une période } \\
\text { de } 3 \text { ans. }\end{array}$ & $\begin{array}{l}\text { L'atteinte la plus marquée est au } \\
\text { niveau du putamen. L'atteinte extra- } \\
\text { striatale est différée dans le temps et } \\
\text { arrive indépendamment de l'atteinte } \\
\text { striatale. La TEP DOPA cérébrale peut } \\
\text { apporter des informations } \\
\text { supplémentaires sur la fonction des } \\
\text { structures mono-aminergiques extra- } \\
\text { striatales. }\end{array}$ \\
\hline Morrish \& Al (1995) & $\begin{array}{l}\text { Patients MP précoces } \\
(n=27) \\
\text { Patients volontaires } \\
\text { sains }(n=16)\end{array}$ & $\begin{array}{l}\text { Etudier l'hypofixation } \\
\text { striatale dès les stades } \\
\text { précoces de la maladie } \\
\text { afin d'évaluer la } \\
\text { capacité de la TEP } \\
\text { DOPA a distinguer les } \\
\text { MP précoces des } \\
\text { patients sains. }\end{array}$ & $\begin{array}{l}\text { Diminution significative de la fixation } \\
\text { putaminale chez les MP } \\
\text { comparativement aux sujets sains de } \\
\text { façon bilatérale. }\end{array}$ \\
\hline
\end{tabular}




\begin{tabular}{|c|c|c|c|}
\hline $\begin{array}{l}\text { Nagasawa \& } \\
\text { (1993) }\end{array}$ & $\begin{array}{l}\text { Patiens MP (n=10) } \\
\text { avec } \\
\text { hémiparkinsonisme } \\
\text { Patients contrôles ( } \mathrm{n}= \\
\text { 10) }\end{array}$ & $\begin{array}{l}\text { Comparer } \\
\text { diminution de la } \\
\text { fixation de la DOPA } \\
\text { entre des patients } \\
\text { présentant un } \\
\text { Parkinson unilatéral et } \\
\text { des patients contrôles } \\
\text { sains. }\end{array}$ & $\begin{array}{l}\text { Diminution significative de la fixation } \\
\text { aussi bien du côté atteint que du côté } \\
\text { pré-symptomatique avec une } \\
\text { correlation avec la sévérité de } \\
\text { l'atteinte. }\end{array}$ \\
\hline Bruke \& Al (2009) & $\begin{array}{l}\text { Patients MP précoces } \\
(n=16) .\end{array}$ & $\begin{array}{l}\text { Etudier la progression } \\
\text { de l'atteinte } \\
\text { dopaminergique } \\
\text { striatale par la TEP } \\
\text { DOPA pendant } 5 \text { ans. }\end{array}$ & $\begin{array}{l}\text { La diminution de la fixation de la DOPA } \\
\text { est non linéaire et plus rapide au début } \\
\text { de la maladie. L'atteinte la plus } \\
\text { marquée est située dans la partie } \\
\text { dorso-caudale du putamen } \\
\text { controlatéral à l'atteinte motrice } \\
\text { prédominante. }\end{array}$ \\
\hline Hilker \& Al (2005) & $\begin{array}{l}\text { MP }(=31) \text {, volontaires } \\
\text { sains }(n=16)\end{array}$ & $\begin{array}{l}\text { Déterminer la } \\
\text { progression de } \\
\text { l'atteinte } \\
\text { dopaminergique dans } \\
\text { la MP grâce à la TEP }\end{array}$ & $\begin{array}{l}\text { Cette étude suggère que le processus } \\
\text { neurodégénératif de la MP suit une } \\
\text { courbe exponentielle négative et } \\
\text { diminue avec l'aggravation des } \\
\text { symptômes dans le temps. }\end{array}$ \\
\hline
\end{tabular}

Tableau 1 : Données de la littérature sur l'apport diagnostic de la TEP cérébrale à la 18 F-FDOPA dans les syndromes parkinsoniens. 


\section{PERFORMANCES DE LA teP A LA 18F-FDOPA DANS LE DIAGNOSTIC DIFFÉRENTIEL DES SYNDROMES PARKINSONIENS}

Selon l'AMM, si la TEP cérébrale à la 18F-FDOPA et le DaTSCAN® permettent de faire le diagnostic différentiel entre maladie de parkinson et tremblement essentiel, psychogène ou iatrogène lié à la prise de neuroleptiques, ces examens ne permettent pas de faire le distinguo entre les différents syndromes parkinsoniens. En effet, au niveau physiopathologique, aucune perte neuronale dopaminergique n'est observée dans les tremblements essentiels, psychogènes ou iatrogènes alors que cette perte neuronale nigro-striée est bien présente pour la paralysie supranucléaire (PSP), l'atrophie multi-systématisée (AMS), la dégénérence cortico-basale (DCB) et la démence à corps de Lewy (DCL) comme dans la MP.

Certaines études se sont attelées à décrire l'aspect de la TEP à la 18F-FDOPA dans ces différentes pathologies. L'équipe de Brooks (18), a décrit un modèle de fixation pour la MP, l'AMS et la PSP en comparant les examens de 16 patients ayant une maladie de parkinson répondant au traitement à 18 patients avec une AMS et 10 patients au diagnostic de PSP. Les patients MP avait une atteinte striatale plus marquée au niveau des putamens postérieurs avec une relative épargne du noyau caudé et présence d'un gradient antéro-postérieur. Les patients PSP avait eux, une atteinte striatale diffuse symétrique, sans différence entre le putamen antérieur, postérieur ou le noyau caudé alors que les patients AMS avaient une atteinte plus hétérogène et moins spécifique, pouvant à la fois toucher l'ensemble du striatum ou mimer une MP avec une atteinte plus marquée au niveau du putamen postérieur. Alors que la diminution de l'intensité de fixation était corrélée au degré de l'atteinte motrice chez les patients MP et AMS, ce n'était pas le cas pour les patients PSP. D'autres études ont aussi confirmé l'impossibilité de distinguer l'AMS de la MP $(30,31)$.

Plus récemment, Ghaemi (32) a montré dans son étude en comparant les données de la TEP à la 18F-FDOPA, au 18F-FDG et au RACLO (11 C-Raclopride) avec celle de l'IRM chez des patients MP, des patients AMS et des patients sains, que la TEP à la 18F-FDOPA ne permettait pas de faire le diagnostic différentiel entre AMS et MP avec une hypofixation striatale et un gradient antéro-postérieur dans les deux cas. La TEP au 18F-FDG et l'IRM permettaient de différencier MP et AMS avec une 
diminution volumétrique striatale et du métabolisme significativement plus marquée dans les AMS. Ces deux derniers examens ne permettaient pas de différencier les patients MP des sujets sains. La TEP-RACLO, récepteurs postsynaptiques de la dopamine, permettait de faire le diagnostic différentiel entre AMS et sujets sains ainsi que entre AMS et MP.

Un schéma de fixation a été décrit pour la DCB avec en TEP à la 18F-FDOPA une réduction de la fixation striatale asymétrique mais homogène sans épargne du noyau caudé (31) et en TEP au 18 FDG une fixation cérébrale asymétrique avec une hypofixation prédominant du même côté que l'atteinte en 18F-FDOPA (33).

Les Parkinsons post-traumatiques ont été étudiés par Turjanski et al (34) qui a comparé la fixation striatale de la 18F-FDOPA chez 6 Parkinsons posttraumatiques à celle de $18 \mathrm{MP}$ et 32 sujets sains. Le schéma de la diminution de fixation de la 18F-FDOPA dans le Parkinson post-traumatique était différent avec une diminution de la fixation globale sans l'épargne relative du noyau caudé.

Enfin, il est recommandé de réaliser une TEP cérébrale à la 18F-FDOPA afin de faire le diagnostic différentiel entre DCL et maladie d'Alzheimer. On retrouvera une hypofixation striatale bilatérale dans la DCL alors que l'examen sera normal pour un Alzheimer (35). Cependant l'examen ne permettra pas de distinguer une DCL d'une MP avec démence (36). 


\begin{tabular}{|c|c|c|c|}
\hline $\begin{array}{l}\text { Etudes (premier } \\
\text { auteur et } \\
\text { année de publication) }\end{array}$ & $\begin{array}{l}\text { Population et } \\
\text { diagnostic } \\
\text { clinique. } \\
\text { Syndromes } \\
\text { parkinsoniens et } \\
\text { patients contrôles }\end{array}$ & Intérêt de l'étude & Résultats principaux \\
\hline Brooks \& Al (2004) & $\begin{array}{l}\text { MP }(n=16), \text { AMS } \\
(n=18), \text { PSP }(=10), \\
\text { volontaires sains } \\
(n=30) .\end{array}$ & $\begin{array}{l}\text { Etudier la fixation de la } \\
\text { FDOPA dans les MP, les } \\
\text { AMS et les PSP en les } \\
\text { comparant à des } \\
\text { volontaires sains } \\
\text { appareillés pour l'âge. }\end{array}$ & $\begin{array}{l}\text { Comparativement aux sujets sains les } \\
\text { MP avaient une diminution de la } \\
\text { fixation du putamen postérieur avec } \\
\text { relative épargne du putamen antérieur } \\
\text { et du noyau caudé, les PSP avaient une } \\
\text { atteinte identique du putamen } \\
\text { antérieur et postérieur et une atteinte } \\
\text { du noyau caudé plus marquée que les } \\
\text { MP. La diminution de la fixation du } \\
\text { noyau caudé et du putamen antérieur } \\
\text { dans les AMS était située entre les MP } \\
\text { et les PSP. }\end{array}$ \\
\hline Ghaemi \& Al (1997) & $\begin{array}{lr}\text { MP } \quad(=24), & \text { AMS } \\
(n=9), & \text { et } \\
\text { volontaires } & \text { sains } \\
(n=7) & \end{array}$ & $\begin{array}{l}\text { Comparer les TEP DOPA, } \\
\text { FDG, RACLO et l'IRM } \\
\text { cérébrale des patients MP, } \\
\text { AMS et contrôles afin } \\
\text { d'affiner le diagnostic } \\
\text { différentiel de ces } \\
\text { pathologies. }\end{array}$ & $\begin{array}{l}\text { La TEP DOPA en tant que marqueur } \\
\text { pré-synaptique ne permet pas de faire } \\
\text { de diagnostic différentiel entre MP et } \\
\text { AMS a l'inverse de l'IRM avec une } \\
\text { diminution du volume, du TEP FDG } \\
\text { qui montre une diminution du } \\
\text { métabolisme et du TEP RACLO comme } \\
\text { récepteur post-synaptiques. }\end{array}$ \\
\hline Turjanski \& Al (1997) & $\begin{array}{l}\text { Syndromes } \\
\text { parkinsoniens post- } \\
\text { traumatiques } \\
(\mathrm{n}=6), \quad \mathrm{MP} \quad \text { et } \\
\text { patients contrôles. }\end{array}$ & $\begin{array}{l}\text { Comparaison des données } \\
\text { de la TEP DOPA chez les } \\
\text { patients sains, MP et } \\
\text { présentant un parkinson } \\
\text { post-traumatique. }\end{array}$ & $\begin{array}{l}\text { La TEP à la 18F-FDOPA permet d'aider } \\
\text { au diagnostic différentiel entre MP et } \\
\text { parkinson post-traumatique en } \\
\text { montrant une atteinte striatale diffuse } \\
\text { alors que les MP ont une relative } \\
\text { épargne du noyau caudé. }\end{array}$ \\
\hline
\end{tabular}




\begin{tabular}{|c|c|c|c|}
\hline Burn \& Al (1994) & $\begin{array}{l}\text { MP }(n=28), \text { AMS } \\
(n=25), \quad \text { PSP }(n= \\
10) \text { et patients } \\
\text { contrôles }(n=27)\end{array}$ & $\begin{array}{l}\text { Evaluation de l'atteinte } \\
\text { dopaminergique striatale } \\
\text { dans la MP, l'AMS et la } \\
\text { PSP par la TEP cérébrale à } \\
\text { la 18F-FDOPA. }\end{array}$ & $\begin{array}{l}\text { La TEP DOPA permet de différencier } \\
\text { les MP des patients contrôles, les MP et } \\
\text { des PSP mais pas des AMS. }\end{array}$ \\
\hline Sawle \& Al (1991) & DCB $(n=6)$ & $\begin{array}{l}\text { Evaluer l'atteinte } \\
\text { dopaminergique des DCB } \\
\text { en TEP DOPA }\end{array}$ & $\begin{array}{l}\text { La DCB en TEP à la 18F-FDOPA montre } \\
\text { une réduction de la fixation striatale } \\
\text { asymétrique mais homogène sans } \\
\text { épargne du noyau caudé }\end{array}$ \\
\hline Laureys \& Al (1999) & $\begin{array}{l}\text { DCB (n = 6), } \\
\text { patients MP }(n=6) \\
\text { et patients } \\
\text { contrôles. }\end{array}$ & $\begin{array}{l}\text { Fixation de la F-FDOPA et } \\
\text { du FDG dans les stades } \\
\text { précoces de DCB } \\
\text { comparativement aux MP. }\end{array}$ & $\begin{array}{l}\text { Hypofixation plus marquée de la } \\
\text { FDOPA au niveau du noyau caudé chez } \\
\text { les DCB, plus marqué du côté } \\
\text { controlatéral à l'atteinte motrice } \\
\text { prédominante. En TEP au } 18 \text { FDG une } \\
\text { fixation cérébrale asymétrique avec } \\
\text { une hypofixation prédominant du } \\
\text { même côté que l'atteinte en 18F- } \\
\text { FDOPA }\end{array}$ \\
\hline Hu \& Al (2000) & $\begin{array}{l}\text { DCL }(n=7) \text {, Maladie } \\
\text { d'Alzheimer } \quad(n= \\
\text { 10) }\end{array}$ & $\begin{array}{l}\text { Diagnostic différentiel } \\
\text { entre la DCL et la maladie } \\
\text { d'Alzheimer } \\
\text { l'utilisation de la } \\
\text { DOPA }\end{array}$ & $\begin{array}{l}\text { La TEP DOPA permet de faire le } \\
\text { diagnostic différentiel entre les deux } \\
\text { pathologies avec une sensibilité de } \\
86 \% \text { et une spécificité de } 100 \% \text { en se } \\
\text { basant sur la fixation du putamen. }\end{array}$ \\
\hline Klein \& Al (2010) & $\begin{array}{l}\text { Patients MP avec } \\
\text { démence }(n=8), \\
\text { DCL }(n=6) \text {, patients } \\
\text { MP sans démence } \\
(n=9) .\end{array}$ & $\begin{array}{l}\text { Comparaison des patients } \\
\text { MP avec démence et des } \\
\text { patients DCL en TEP } \\
\text { cérébrale avec utilisation } \\
\text { de différents traceurs. }\end{array}$ & $\begin{array}{l}\text { Pas de différence significative trouvée } \\
\text { entre les patients MP avec démence et } \\
\text { les patients présentant une DCL } \\
\text { quelque soit le radiotraceur utilisé } \\
\text { (FDG, FDOPA et N-11-C-methyl-4- } \\
\text { piperidyl acetate). }\end{array}$ \\
\hline
\end{tabular}

Tableau 2 : Données de la littérature sur le diagnostic différentiel des syndromes parkinsoniens par la TEP cérébrale à la 18F-FDOPA. 


\section{CONCLUSION ET DISCUSSION DE LA REVUE DE LA LITTÉRATURE}

Notre revue de littérature était initialement axée sur la TEP cérébrale à la 18F-FDOPA dans le diagnostic des syndromes parkinsoniens en routine clinique mais il existe peu de revues de la littérature sur le sujet et la plupart des articles concernent la recherche. Nous avons trouvé un seul article publié par Puñal-Riobóo et al (37) qui s'approche de notre sujet avec une revue de la littérature sur l'utilité clinique de la TEP cérébrale à la 18F-FDOPA dans les mouvements anormaux. Nous retenons de notre recherche que la TEP cérébrale à la 18 F-FDOPA possède des performances diagnostiques suffisantes au moins équivalentes à celles du DaTSCAN® dès les stades précoces de la pathologie. Certains articles ont pourtant émis l'hypothèse que par la présence de mécanismes compensateurs, la 18F-FDOPA était un radiotraceur qui ne permettait pas un diagnostic aussi précoce que les ligands du DAT mais cela n'est pas ressorti de façon évidente dans notre recherche. En modélisant la maladie de Parkinson chez le primate, Langstone et al (38) ont montré, en 1984, qu'une perte des neurones dopaminergiques nigro-striés d'environ 60 à 70\% est nécessaire pour voir apparaître des signes cliniques et on peut donc penser que l'examen d'imagerie initial (TEMP ou TEP) pourra mettre en évidence la perte neuronale dopaminergique. L'apparition tardive des symptômes sous-tend l'existence de mécanismes compensateurs du dysfonctionnement pré et postsynaptique dopaminergique pendant la phase pré-symptomatique de la MP qui seraient dépassés lorsque les symptômes apparaissent comme l'évoque l'article de Ribeiro et al (39) ou encore celui de Nandhagopal et al (40). Les mécanismes compensateurs évoqués sont l'augmentation de la synthèse de dopamine par les neurones restants par le biais de la tyrosine hydroxylase et de la dopadécarboxylase up régulation, la diminution de l'élimination de la dopamine par la down régulation du DAT (le transporteur pré-synaptique de la dopamine) et une augmentation du nombre de récepteurs D2 post-synaptiques. En effet Ribeiro et al a comparé les performances de la TEP à la 18 F-FDOPA à celle d'un ligand DAT : le 76Br-fluorethyl-methyl-carboxymethoxy-bromophényl-tropane (76Br-FE-CBT) sur 18 patients (10 MP précoces et 8 MP avancées) ; l'équipe a trouvé que chez les MP précoces, la fixation de ce dernier était de $43 \%$ de la fixation des patients contrôles, contre une fixation de la 18 F-FDOPA à $63 \%$ de la fixation des patients contrôles. 
Cette différence n'était pas retrouvée chez les MP avancés. L'équipe de Lee (41) comme celle de Nandhagopal ont montré ces mécanismes compensateurs au début de la maladie en comparant trois radiopharmaceutiques pré-synaptiques : le 11Cdihydrotetrabenzamine (11C-DTBZ) traceur de VMAT2, le 11C-methylphénidate (11C-MP) traceur du DAT et la 18F-FDOPA et les seuils de fixation de ce dernier radiopharmaceutique étaient également les plus élevés. Cependant, Nandhagopal a fait une étude prospective avec un suivi dans le temps et a montré que ces effets compensateurs disparaissent après 2 à 5 ans d'évolution de la maladie. On pourrait penser que ces effets compensateurs diminuent la sensibilité de la TEP à la 18FFDOPA mais comme nous l'avons montré plus haut dans notre revue de la littérature, la plupart des études calculant la sensibilité et la spécificité de notre examen, ne confirme pas cette supposition et notamment l'étude comparative de Eshuis et al (27) qui fait une comparaison directe entre la TEP cérébrale à la 18FFDOPA et le DaTSCAN $®$ et qui trouve des performances au moins similaires pour les deux examens. 


\section{ETUDE CLINIQUE PROSPECTIVE}

Les données de la littérature sur l'intérêt clinique de la TEP cérébrale à la 18F-FDOPA sont assez pauvres et c'est ce que nous avons tenté d'évaluer dans cette étude par le biais d'un questionnaire envoyé aux médecins prescripteurs de l'examen. Ensuite en s'appuyant sur le diagnostic final retenu par le neurologue, nous avons évalué notre interprétation visuelle ainsi que l'analyse semi-quantitative et tenté de définir des seuils diagnostiques fiables.

\section{I/ MATÉRIEL ET MÉTHODES}

\section{A. POPULATION}

Notre étude prospective a été réalisée sur une période de six mois et demi, du 15 octobre 2014 au 29 avril 2015. Pendant cette période, pour tous les patients venant dans notre service passer une TEP cérébrale à la 18 F-FDOPA pour l'exploration d'un syndrome parkinsonien atypique, un questionnaire (annexe 1) a été envoyé avec les résultats de l'examen au médecin neurologue prescripteur. Parallèlement, en interprétant l'examen, le médecin nucléaire sénior remplissait lui une fiche d'interprétation (annexe 2) prenant en compte l'interprétation visuelle et des mesures semi-quantitatives. Afin de vérifier la reproductibilité de l'interprétation, un second médecin nucléaire junior a relu et refait les analyses semi-quantitatives en aveugle sur un échantillon d'examen tiré au sort.

En se basant sur le diagnostic final retenu par le médecin neurologue nous avons défini deux groupes de patients : les sujets dits « normaux » c'est-à-dire sans atteinte dopaminergique et les sujets dits « anormaux » c'est-à-dire qui présentaient une atteinte dopaminergique (maladie de parkinson et syndrome extra-pyramidal atypique). C'est le diagnostic final du neurologue qui nous sert de référence. 


\section{B. PARAMÈTRES D'ACQUISITION TEP/TDM}

\section{Préparation du patient}

Comme le recommande la Société Française de Médecine Nucléaire (SFMN) (42), tout traitement anti-parkinsonien contenant de la dopa était arrêté au minimum 12 heures avant l'examen. Il était demandé au patient de faire un jeûne protéique 4 à 6 heures avant l'examen afin d'éviter toute compétition entre des acides aminés exogènes et l'acide-aminé marqué.

Une heure avant l'injection du radiotraceur, $100 \mathrm{mg}$ de Carbidopa (inhibiteur de la dopa décarboxylase périphérique) étaient administrés au patient par voie orale en l'absence de contre-indication afin d'augmenter la biodisponibilité cérébrale de la 18F-FDOPA.

\section{Réalisation de l'examen}

Quatre-vingt-dix minutes après l'injection intra-veineuse du radiopharmaceutique (18F-FDOPA) à la dose de $2 \mathrm{MBq} / \mathrm{kg}$, était réalisé un topogramme de repérage, une tomodensitométrie (nécessaire à la correction d'atténuation) suivie des acquisitions TEP (tableau 3). Le patient était positionné en décubitus dorsal, les bras le long du corps, la tête positionnée et sanglée dans une têtière. 


\begin{tabular}{|c|c|c|}
\hline & TDM & TEP \\
\hline Acquisitions & $\begin{array}{l}100 \mathrm{kV} \\
100 \mathrm{mAs} \text { (valeur moyenne; } \\
\text { système CAREDOSE) }\end{array}$ & $\begin{array}{l}\text { Images dynamiques 3D (mode liste) } \\
1 \text { pas cérébral } \\
\text { Durée des acquisitions : } 10 \text { minutes }\end{array}$ \\
\hline Reconstructions & $\begin{array}{l}\text { Axiales } \\
1 \mathrm{~mm} \times 0,7 \mathrm{~mm} \\
\text { Filtre de convolution } \mathrm{H} 31 \mathrm{~S} \\
\text { moyen lisse+ } \\
\text { Champ de vue } 250 \mathrm{~mm} \text {. }\end{array}$ & $\begin{array}{l}\text { Reconstruction itérative OSEM (5 } \\
\text { itérations ; } 24 \text { sous-ensembles); } \\
\text { Matrice } 400 * 400 \\
\text { Filtre gaussien } 4 \mathrm{~mm} \text {; } \\
\text { Images corrigées de l'atténuation et la } \\
\text { diffusion. } \\
\text { Pas de correction de résolution } \\
\text { spatiale }\end{array}$ \\
\hline $\begin{array}{l}\text { Affichage } \\
\text { images }\end{array}$ & $\begin{array}{l}\text { Fenêtre cérébrale } \\
(\mathrm{W}=80 \mathrm{UH}, \mathrm{L}=40 \mathrm{UH}) .\end{array}$ & $\begin{array}{l}\text { Plan CA-CP } \\
\text { Normalisation : striatum } \\
\text { Echelle de couleur linéaire type « arc- } \\
\text { en-ciel ». }\end{array}$ \\
\hline
\end{tabular}

Tableau 3 : Paramètres d'acquisitions, de reconstructions et d'affichage des images TEP/TDM

\section{ANALYSE DES IMAGES}

Les données TEP brutes ont été reconstruites et corrigées de l'atténuation grâce à la cartographie par tomodensitométrie (TDM) des coefficients massiques $(\mu)$ d'atténuation. L'affichage des images se faisait dans le plan axial, étape préalable indispensable dont la qualité de réalisation conditionne la quantification, et était réalisé après alignement du plan des commissures antérieures et postérieures (CACP) obtenu sur un plan de coupe sagittal et en s'assurant du positionnement symétrique des deux rochers dans le plan frontal. Pour les VOIs du striatum et du putamen antérieur et postérieur, leur bon positionnement était également vérifié sur un plan sagittal respectivement ré axé sur le noyau caudé puis sur le putamen. 
La quantification du métabolisme dopaminergique striatal spécifique a été réalisée à partir de mesures recueillies dans les volumes d'intérêt (VOI) droits et gauches suivants (Figure 8):

- VOI striata défini par une méthode d'isocontour dont le seuil était arbitrairement choisi par l'observateur, de telle manière que la VOI englobe la totalité d'un striatum (Figure 8 : sphère jaune).

- VOI du noyau caudé : sphère de $2 \mathrm{~cm}^{3}$ (méthode manuelle) (Figure 8 : sphère grise).

- VOI des putamens antérieur et postérieur: sphères de $2 \mathrm{~cm}^{3}$ (méthode manuelle) (Figure 8 : sphères verte et orange).

La fixation non spécifique de la 18F-FDOPA c'est-à-dire le bruit de fond était analysée sur un VOI sphérique de $2 \mathrm{~cm}^{3}$ placé dans le cortex occipital (Figure 8 : sphère blanche).

Dans chaque VOI, la quantification de l'activité a été réalisée à partir des mesures du SUV (standardized uptake value ; sans unité), selon la définition suivante :

$$
S U V=\frac{\text { Concentration de la 18FDOPA dans une VOI donnée }(\mathrm{Bq} / \mathrm{L})}{\text { Activité injectée de 18FDOPA }(\mathrm{Bq}) * \text { masse du patient }(\mathrm{kg})}
$$

Seul le SUV max (fixation maximale de 18F-FDOPA au sein du VOI donné) a été mesuré et pris en compte dans notre analyse statistique. En effet lors d'une précédente étude (12) où le SUV mean (SUV moyen) avait aussi été étudié nous avions trouvé ce paramètre insuffisamment robuste pour quantifier le métabolisme dopaminergique striatal. En effet le SUV max apparaît plus reproductible en routine clinique pour quantifier la neurotransmission dopaminergique striatale.

Une approximation du potentiel de liaison (PL, traduisant l'activité métabolique dopaminergique spécifique) a été définie pour chaque VOI selon les ratios suivants :

- SOR (max) : SUV (max) striatal / SUV occipital

- COR (max) : SUV (max) caudé / SUV occipital

- POR antérieur (max) : SUV (max) putamen antérieur / SUV occipital

- POR postérieur (max) : SUV (max) putamen postérieur/ SUV occipital 
La définition des VOI et le recueil des SUV ont été réalisés à l'aide de la console de traitement d'images SyngoVia ${ }^{\circledR}$, Siemens, utilisée en routine clinique.
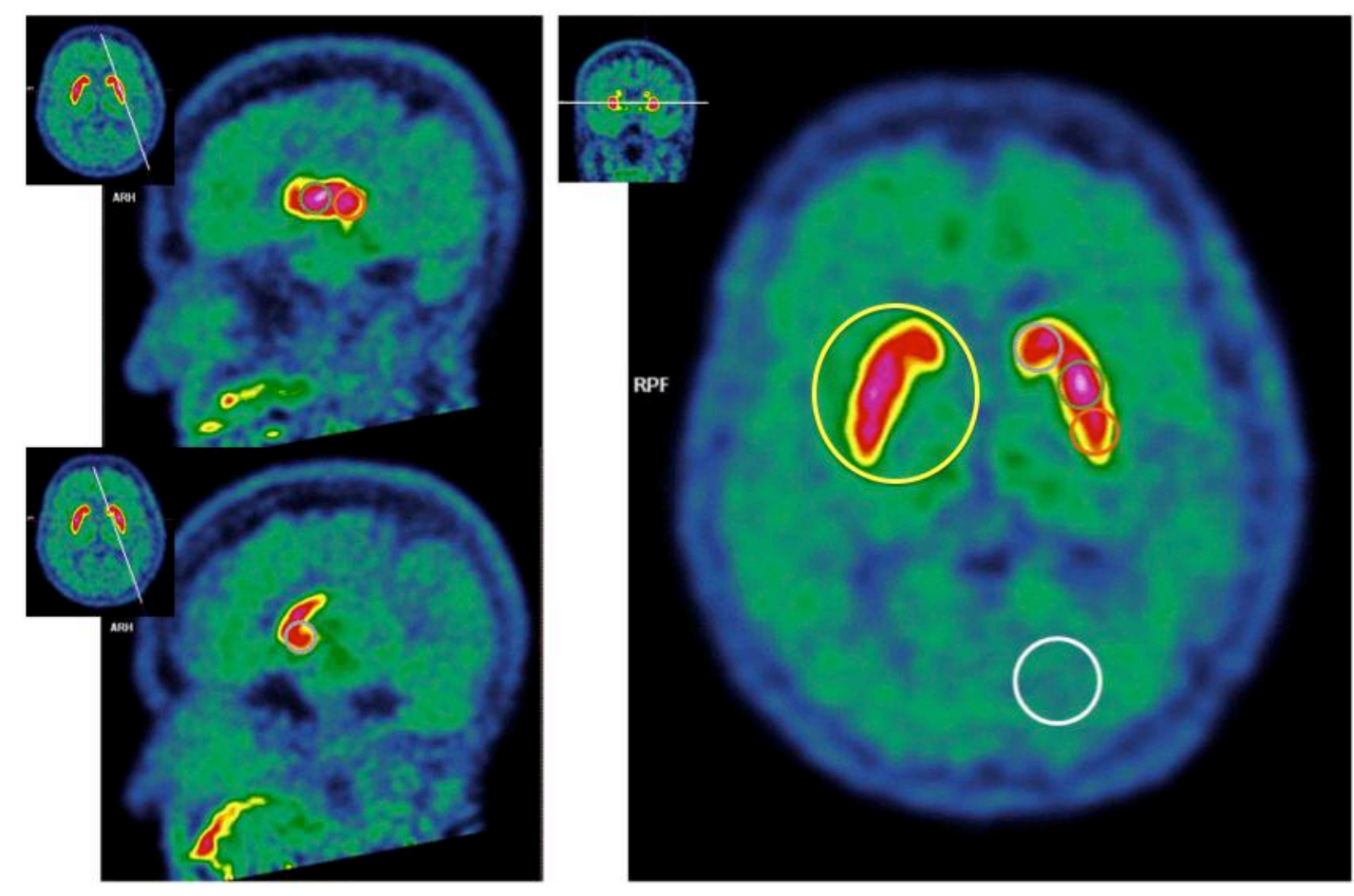

Figure 8 : Réalisation des VOI en coupe axiale plan CA-CP et sagittale ré axée dans le plan du putamen puis du noyau caudé. Sphère jaune : VOI striatal. Sphère grise: VOI noyau caudé. Sphère verte: VOI putamen antérieur. Sphère orange: VOI putamen postérieur. Sphère blanche: VOI occipitale.

\section{QUESTIONNAIRE}

Le questionnaire (annexe 1) remis avec les résultats de l'examen de chaque patient au médecin neurologue prescripteur avait pour but d'évaluer l'apport clinique de l'examen et l'aide au diagnostic ainsi qu'à la prise en charge du patient. Le médecin neurologue pouvait y répondre en s'appuyant sur le résultat de l'examen TEP cérébrale à la 18 F-FDOPA puis le renvoyer par courrier dans notre service. Le questionnaire a été élaboré avec l'aide de médecins nucléaires, de neurologues ainsi que d'un médecin de santé publique, statisticien. Le questionnaire comprenait 10 questions à choix multiples reparties en 3 volets :

- le premier volet sur l'indication de l'examen et la clinique du patient, 
- le deuxième volet sur le diagnostic, l'hypothèse diagnostique avant l'examen, le diagnostic final retenu ainsi que la corrélation entre l'aspect clinique et le résultat TEP,

- le troisième volet visant à évaluer l'intérêt de la TEP dans l'aide au diagnostic, la prise en charge du patient et son suivi.

\section{E. ANALYSE STATISTIQUE}

Les analyses descriptives ont été réalisées pour l'ensemble des variables de l'étude. Les variables catégorielles ont été décrites par effectifs et pourcentages. Les variables quantitatives par moyennes et écarts types. Selon les besoins de l'analyse, certaines variables quantitatives ont pu être catégorisées en fonction de leur dispersion (médiane, terciles ou quartiles).

Nous avons également regroupé les modalités des variables diagnostic initial et diagnostic final en 3 catégories: "maladie de Parkinson », "syndrome extrapyramidal » et «absence d'atteinte dopaminergique » et utilisé ces nouvelles variables selon les nécessités de l'analyse.

Des analyses univariées ont été réalisées. Les variables catégorielles ont été étudiées par test du Khi 2 ou test exact de Fisher lorsque les conditions d'application du Khi2 n'étaient pas remplies. Les comparaisons de moyennes ont été réalisées par test $t$ de Student pour échantillons indépendants ou pour échantillons appareillés selon les situations. Pour chaque variable quantitative la normalité de la distribution était vérifiée par test de Kolmogorov-Smirnov et visuellement par normogramme QQ. Lorsque les conditions d'application du test t de Student n'étaient pas remplies, nous avons eu recours au test non paramétrique de Mann Whitney.

Pour vérifier la reproductibilité inter-opérateur de l'interprétation de l'examen, un tirage au sort aléatoire a été réalisé pour obtenir 30 examens qui ont été relus et analysés par un opérateur différent qui n'avait pas accès aux informations cliniques ni à l'interprétation et aux résultats de l'analyse initiale. La reproductibilité a été évaluée sur les données des SUV par coefficient de corrélation intra-classe. Ce coefficient prend des valeurs théoriques comprises entre 0 et 1 . Plus sa valeur est proche de 1 meilleur est l'accord entre 2 observateurs. 
Nous avons apprécié les qualités diagnostiques par sensibilité et spécificité. Pour chaque outil de mesure la sensibilité et la spécificité qui minimisent le nombre de faux positifs et de faux négatifs ont été déterminées par la méthode de Youden. Des courbes ROC (Receiving Operating Characteristics) ont été produites pour chaque outil diagnostique. Les sensibilités, spécificités et AUC (aire sous la courbe) ont été calculées et exprimées avec intervalle de confiance à 95\%. Les comparaisons des AUC des différents outils diagnostiques ont été réalisées selon la méthode décrite par De Long et al (43). Les tests ont été réalisés de façon bilatérale avec un risque alpha fixé à 0,05 .

Les analyses ont été réalisées avec le logiciel PASW 18.0. Les analyses concernant les qualités diagnostiques des outils de mesures ont été effectuées par PASW 18.0 et MedCalc 15.6.1. 


\section{II/ RÉSULTATS}

\section{A. RÉSULTATS DE L'ÉVALUATION CLINIQUE}

Pendant une période de 6 mois et demi nous avons interrogé par le biais d'un questionnaire les médecins neurologues qui nous ont adressé des patients pour réaliser une TEP cérébrale à la 18F-FDOPA. Notre but était d'évaluer l'intérêt clinique et diagnostique de notre examen dans la prise en charge du patient présentant un syndrome extra-pyramidal.

\section{Réponses au questionnaire}

Nous avons reçu finalement 91 réponses aux 192 questionnaires envoyés soit un taux de réponse de 47\%. La figure 9 illustre l'étape de récupération des questionnaires.

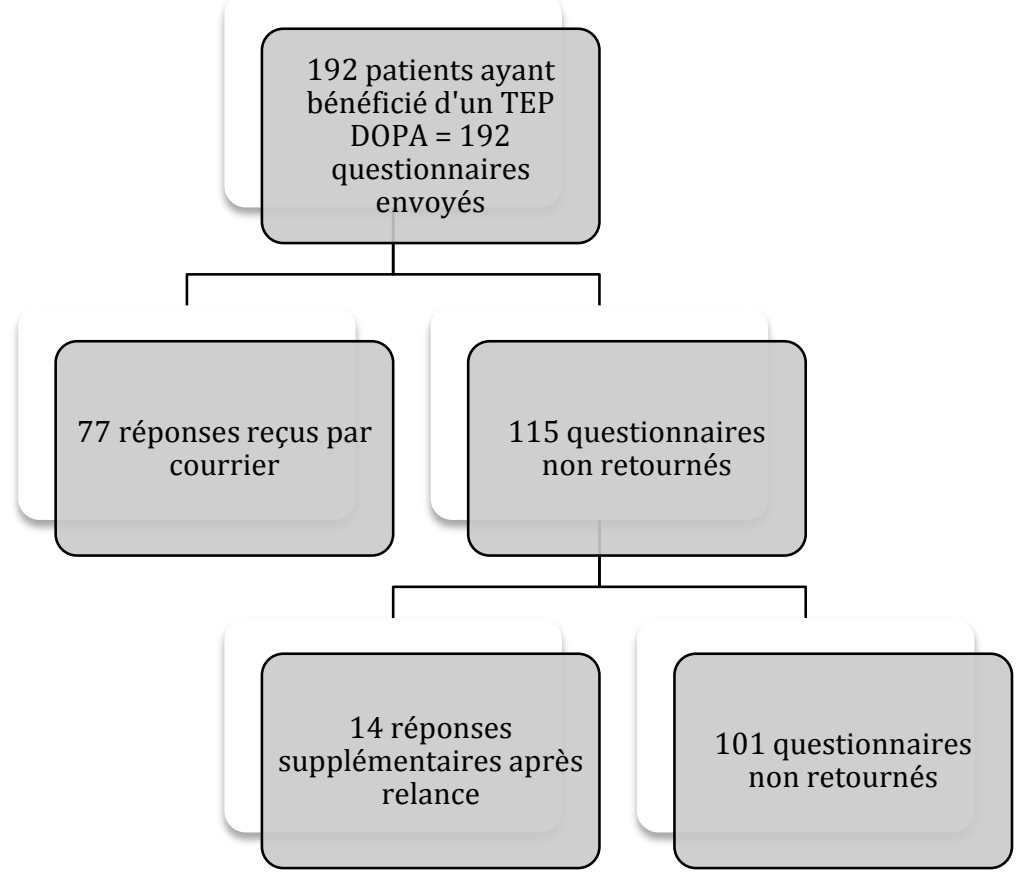

Figure 9 : Réponses au questionnaire 


\section{Médecins participants}

Les 91 réponses ont été obtenues de la part de 16 neurologues. Leurs caractéristiques sont les suivantes : 9 hommes et 7 femmes, 4 neurologues avaient une activité mixte (ville et structure hospitalière), 8 neurologues exerçaient uniquement en cabinet de ville et 4 neurologues étaient des médecins hospitaliers.

Le taux de réponse par neurologue est variable et décrit figure 10. Parmi eux, 3 médecins ont plus activement répondu au questionnaire; le premier répondeur (30,8\% des réponses) avait une activité mixte, le second (22\% des réponses) une activité hospitalière et le troisième $(13,2 \%$ des réponses) une activité de ville exclusive.

\section{Taux de réponse par médecin}
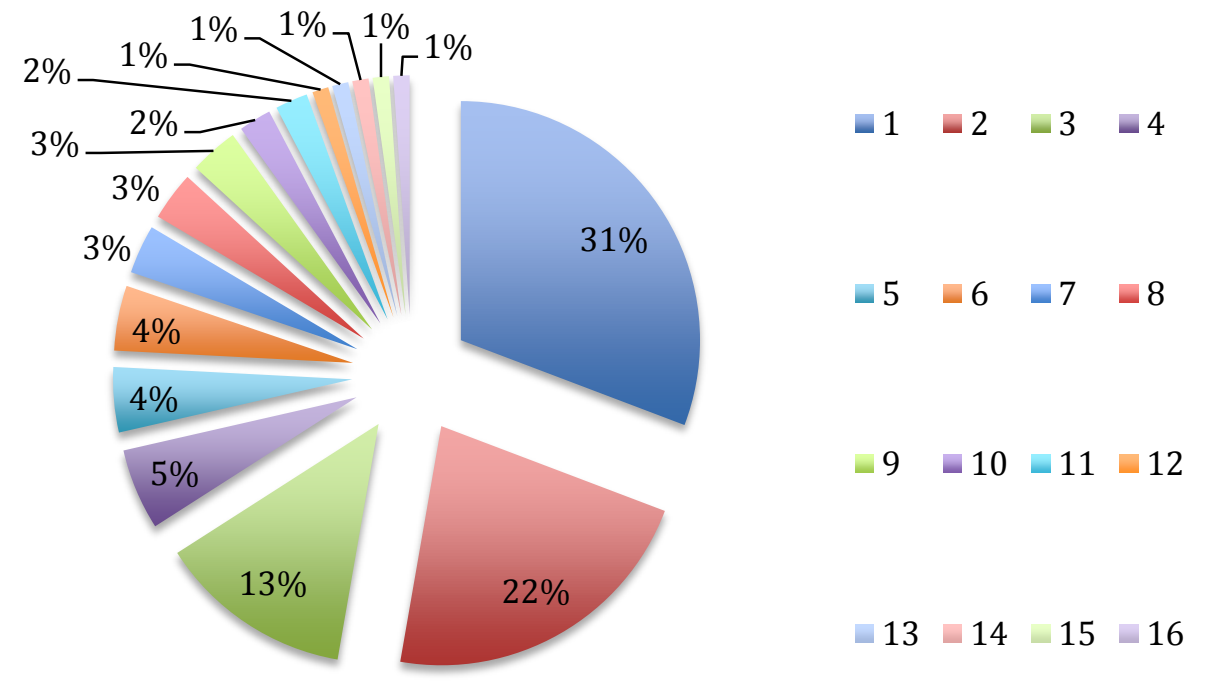

Figure 10 : Taux de réponse par médecin 


\section{Caractéristiques de la population des patients}

\begin{tabular}{|c|c|c|c|}
\hline & & $\mathrm{n}$ & Pourcentage (\%) \\
\hline \multicolumn{2}{|l|}{ Patient } & 91 & 100 \\
\hline \multicolumn{2}{|l|}{ Homme } & 50 & 55 \\
\hline \multicolumn{2}{|l|}{ Femme } & 41 & 45 \\
\hline \multirow[t]{2}{*}{ Age } & Moyen & 71,4 & \\
\hline & Médian & 73,4 & \\
\hline \multirow[t]{5}{*}{ Latéralisation des symptômes } & Droite & 6 & 7 \\
\hline & Gauche & 15 & 16,5 \\
\hline & Droite $>$ Gauche & 13 & 14 \\
\hline & Gauche $>$ Droite & 25 & 27,5 \\
\hline & Bilatérale & 31 & 34 \\
\hline \multirow[t]{4}{*}{ Durée des symptômes } & $<6$ mois & 14 & 15 \\
\hline & 6 mois -2 ans & 38 & 42 \\
\hline & 2 ans -5 ans & 28 & 31 \\
\hline & $>5$ ans & 11 & 12 \\
\hline
\end{tabular}

Tableau 4 : Description de la population

Les caractéristiques de la population de patient sont exposées dans le tableau 4. Nous avons 91 patients, dont 50 hommes (55\%) et 41 femmes (45\%). L'âge moyen de la population était de 71,4 ans et l'âge médian de 73,4. Le patient le plus jeune avait 43 ans et le plus âgé 89 ans.

La latéralisation des symptômes moteurs ainsi que la durée d'évolution des symptômes au moment de la prescription de l'examen sont détaillés dans le tableau 4.

Environ $70 \%$ des patients $(n=64)$ avaient également une symptomatologie non motrice associée à la symptomatologie motrice. Le symptôme non moteur le plus fréquent était les troubles cognitifs $(29 \%, n=26)$, venaient ensuite les troubles de l'équilibre $(23 \%, n=21)$, la dépression $(21 \%, n=19)$, la douleur $(11 \%, n=10)$ et plus rarement la dysautonomie $(2 \%, \mathrm{n}=2)$. 


\section{Evaluation du cheminement diagnostique avant et après la TEP}

L'atteinte de la voie dopaminergique selon le neurologue avant la prescription de l'examen était :

- très probable dans $16,5 \%$ des cas $(\mathrm{n}=15)$,

- probable dans $37,4 \%$ des cas ( $n=34)$,

- peu probable dans $42,9 \%$ des cas $(n=39)$,

- improbable dans $3,3 \%$ des cas ( $n=3)$.

Les figures 11 et 12 décrivent les hypothèses diagnostiques avant la TEP puis les diagnostics retenus après la TEP. Avant la TEP, les neurologues avaient deux ou trois hypothèses diagnostiques pour un tiers des patients $(n=31)$ et une seule hypothèse dans les autres cas ( $\mathrm{n}=60)$.

Parmi les hypothèses diagnostiques et les diagnostics retenus il était proposé : la MP précoce, la MP avancée, l'AMS, la DCB, le parkinson vasculaire, la DCL, la PSP, un syndrome extra-pyramidal atypique, un syndrome extra-pyramidal d'origine iatrogène, un tremblement essentiel, ou d'origine psychogène. Enfin il était possible de formuler en toute lettre une autre hypothèse ou un autre diagnostic. Parmi les autres diagnostics, il a été formulé, deux maladies d'Alzheimer, une démence vasculaire, deux démences non étiquetées, deux démences fronto-temporales, une hydrocéphalie à pression normale, une origine post-radique/post-lésionnelle et deux tremblements dystoniques. Les praticiens ont aussi formulé en toute lettre «ne sait pas » pour le diagnostic final retenu dans 6 cas et une seule fois le médecin n'a conclu à aucun diagnostic. Dans ces 7 derniers cas où le neurologue n'a pas pu conclure à un diagnostic final, une TEP à la 18F-FDOPA a été demandé pour suivre l'évolution dans 2 cas.

Les neurologues ont trouvé que leur examen clinique était cohérent avec le résultat de la TEP dans $78 \%$ des cas $(n=71 / 91)$. 


\section{Hypothèse diagnostique avant TEP}

- MP précoce

- MP avancée

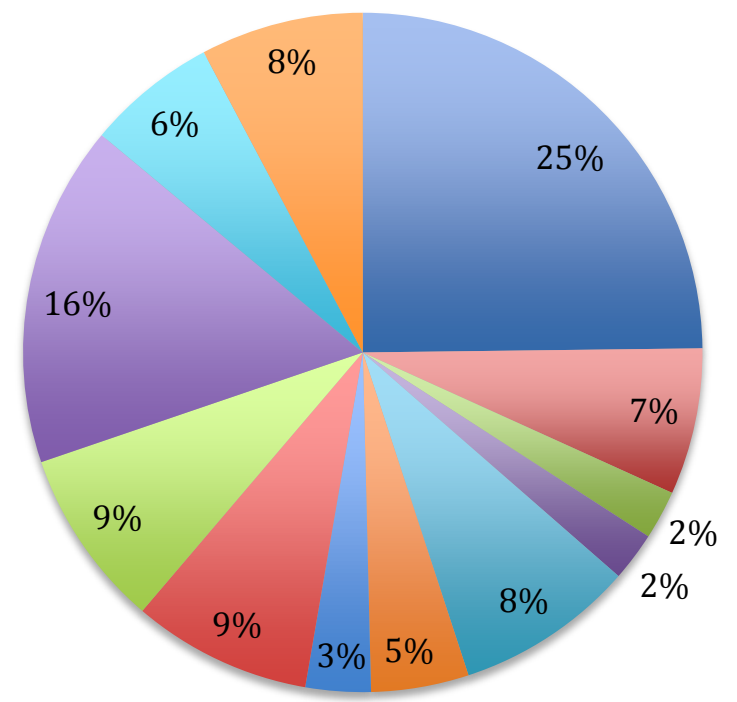

$\square \mathrm{AMS}$

- DCB

- ParkVaculaire

$\square$ DCL

- PSP

— SextPyr atyp

च Iatrogénie

- TremblEssent

OriginePsych

$\square$ Autre

Figure 11 : Hypothèse diagnostique avant TEP

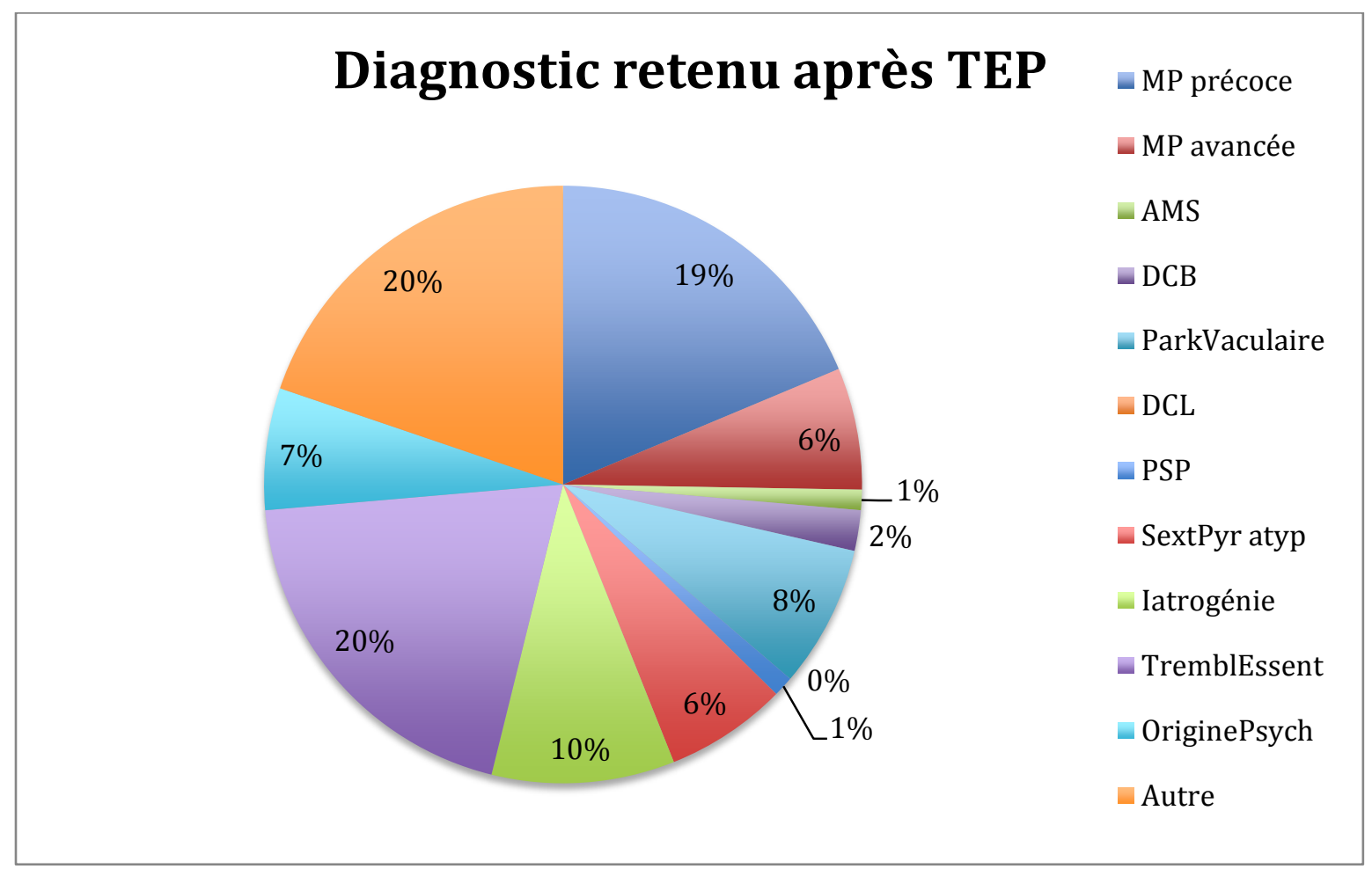

Figure 12 : Diagnostic retenu après TEP 
- Lorsque le médecin neurologue suspectait une maladie de parkinson précoce ou avancée le diagnostic retenu était modifié dans $1 / 3$ des cas $(n=9 / 29)$.

- Lorsque le diagnostic suspecté était un autre syndrome extra-pyramidal, le diagnostic retenu après la TEP était modifié dans plus d'un cas sur deux $(n=15 / 27)$.

- Lorsque le médecin neurologue ne suspectait pas d'atteinte dopaminergique, la TEP était effectivement normale dans $97 \%$ des cas $(n=34 / 35)$.

Les hypothèses diagnostiques avant la TEP cérébrale à la 18 F-FDOPA ainsi que le diagnostic final retenu par le médecin neurologue sont décrites pour chaque patient dans le tableau 5 afin de donner plus de visibilité aux résultats.

- Pour 66 patients (72,5\%) l'hypothèse diagnostique initiale correspondait au diagnostic final retenu.

- Pour 25 patients (27,5\%), l'hypothèse diagnostique initiale était différente du diagnostic final, autrement dit la TEP a permis de changer le diagnostic pour près de 1 patient sur $3(25 / 91)$.

\begin{tabular}{|c|c|c|c|}
\hline $\begin{array}{l}\text { Patient } \\
\text { (num.) }\end{array}$ & Hypothèse(s) diagnostique(s) initiale(s) & Diagnostic final retenu & $\begin{array}{l}\text { Aide au } \\
\text { diagnostic }\end{array}$ \\
\hline 1 & DFT & DFT & renforce \\
\hline 2 & iatrogénie & iatrogénie & renforce \\
\hline \multirow{2}{*}{3} & tremblement essentiel & \multirow{2}{*}{ tremblement essentiel } & \multirow[b]{2}{*}{ confirme } \\
\hline & MP précoce & & \\
\hline \multirow{2}{*}{4} & tremblement essentiel & \multirow{2}{*}{ MP précoce } & \multirow[b]{2}{*}{ confirme } \\
\hline & MP précoce & & \\
\hline \multirow{2}{*}{5} & psychogène & \multirow{2}{*}{ MP avancée } & \multirow[b]{2}{*}{ confirme } \\
\hline & MP avancée & & \\
\hline 6 & sd extrapyramidal atypique & psychogène & élimine \\
\hline 7 & PSP & PSP & renforce \\
\hline \multirow{2}{*}{8} & tremblement essentiel & \multirow{2}{*}{ tremblement essentiel } & \multirow[b]{2}{*}{ élimine } \\
\hline & MP précoce & & \\
\hline
\end{tabular}




\begin{tabular}{|c|c|c|c|}
\hline 9 & sd extrapyramidal atypique & sd extrapyramidal atypique & confirme \\
\hline 10 & MP précoce & MP précoce & confirme \\
\hline 11 & MP précoce & MP précoce & confirme \\
\hline 12 & autre & autre, ne sait pas & élimine \\
\hline 13 & MP précoce & MP précoce & confirme \\
\hline 14 & iatrogénie & iatrogénie & $\begin{array}{l}\text { confirme et } \\
\text { élimine }\end{array}$ \\
\hline 15 & iatrogénie & iatrogénie & $\begin{array}{l}\text { renforce et } \\
\text { confirme }\end{array}$ \\
\hline 16 & tremblement essentiel & tremblement essentiel & renforce \\
\hline 17 & MP précoce & MP précoce & confirme \\
\hline 18 & parkinson vasculaire & parkinson vasculaire & renforce \\
\hline 19 & MP précoce & tremblement essentiel & renforce \\
\hline 20 & MP précoce & MP précoce & confirme \\
\hline 21 & sd extrapyramidal atypique & sd extrapyramidal atypique & confirme \\
\hline 22 & MP précoce & MP précoce & confirme \\
\hline \multirow{2}{*}{23} & tremblement essentiel & \multirow{2}{*}{ tremblement essentiel } & \multirow[b]{2}{*}{ renforce } \\
\hline & MP précoce & & \\
\hline 24 & MP précoce & tremblement essentiel & élimine \\
\hline 25 & parkinson vasculaire & parkinson vasculaire & confirme \\
\hline 26 & MP précoce & MP précoce & renforce \\
\hline 27 & tremblement essentiel & tremblement essentiel & renforce \\
\hline \multirow{2}{*}{28} & AMS & \multirow{2}{*}{ MP précoce } & \multirow[b]{2}{*}{ renforce } \\
\hline & MP précoce & & \\
\hline 29 & DCB & DCB & élimine \\
\hline \multirow{2}{*}{30} & psychogène & \multirow{2}{*}{ MP avancée } & \multirow[b]{2}{*}{ renforce } \\
\hline & MP avancée & & \\
\hline 31 & MP précoce & MP précoce & renforce \\
\hline
\end{tabular}




\begin{tabular}{|c|c|c|c|}
\hline \multirow{2}{*}{32} & MP avancée & \multirow{2}{*}{ HPN } & \multirow[b]{2}{*}{ confirme } \\
\hline & HPN & & \\
\hline 33 & tremblement essentiel & tremblement essentiel & renforce \\
\hline 34 & MP avancée & psychogène & renforce \\
\hline 35 & PSP & $\begin{array}{l}\text { troubles de la marche } \\
\text { d'origine multifactorielle } \\
\text { (neuropathie périphérique, } \\
\text { HPN) }\end{array}$ & renforce \\
\hline 36 & tremblement essentiel & tremblement essentiel & confirme \\
\hline 37 & sd extrapyramidal atypique & sd extrapyramidal atypique & renforce \\
\hline 38 & sd extrapyramidal atypique & tremblement essentiel & renforce \\
\hline \multirow{2}{*}{39} & psychogène & \multirow{2}{*}{ psychogène } & \multirow[b]{2}{*}{ confirme } \\
\hline & iatrogénie & & \\
\hline 40 & parkinson vasculaire & parkinson vasculaire & confirme \\
\hline 41 & MP précoce & MP précoce & confirme \\
\hline 42 & $\mathrm{DCL}$ & DFT & élimine \\
\hline 43 & DCB & sd extrapyramidal atypique & élimine \\
\hline 44 & sd extrapyramidal atypique & sd extrapyramidal atypique & renforce \\
\hline \multirow{2}{*}{45} & iatrogénie & \multirow{2}{*}{ psychogène } & \multirow[b]{2}{*}{ élimine } \\
\hline & psychogène & & \\
\hline \multirow{3}{*}{46} & tremblement essentiel & \multirow{3}{*}{ n'a pas conclu } & \multirow[b]{3}{*}{ élimine } \\
\hline & iatrogène & & \\
\hline & MP précoce & & \\
\hline \multirow{2}{*}{47} & sd extrapyramidal atypique & \multirow{2}{*}{ MP avancée } & \multirow[b]{2}{*}{ confirme } \\
\hline & MP avancée & & \\
\hline 48 & MP précoce & MP précoce & $\begin{array}{l}\text { confusion et } \\
\text { rien }\end{array}$ \\
\hline 49 & parkinson vasculaire & démence de type Alzheimer & élimine \\
\hline
\end{tabular}




\begin{tabular}{|c|c|c|c|}
\hline & $\mathrm{DCL}$ & & \\
\hline 50 & autre & autre, ne sait pas & élimine \\
\hline 51 & tremblements dystoniques & tremblements dystoniques & renforce \\
\hline 52 & tremblement essentiel & tremblement essentiel & $\begin{array}{l}\text { renforce et } \\
\text { confirme }\end{array}$ \\
\hline 53 & parkinson vasculaire & parkinson vasculaire & confirme \\
\hline \multirow{2}{*}{54} & Alzheimer démence vasculaire & \multirow[t]{2}{*}{ Alzheimer démence } & \multirow[b]{2}{*}{ élimine } \\
\hline & $\mathrm{DCL}$ & & \\
\hline 55 & parkinson vasculaire & parkinson vasculaire & confirme \\
\hline \multirow{2}{*}{56} & parkinson vasculaire & \multirow{2}{*}{ démence non spécifique } & \multirow[b]{2}{*}{ élimine } \\
\hline & démence non spécifique & & \\
\hline \multirow{2}{*}{57} & tremblement essentiel & \multirow{2}{*}{ tremblement essentiel } & \multirow[b]{2}{*}{ renforce } \\
\hline & MP précoce & & \\
\hline 58 & MP précoce & MP précoce & confirme \\
\hline 59 & parkinson vasculaire & parkinson vasculaire & renforce \\
\hline 60 & sd extrapyramidal atypique & parkinson vasculaire & renforce \\
\hline \multirow{2}{*}{61} & MP précoce & \multirow{2}{*}{ tremblement essentiel } & \multirow[b]{2}{*}{ confirme } \\
\hline & tremblement essentiel & & \\
\hline 62 & $\mathrm{DCL}$ & démence non étiquetée & rien \\
\hline \multirow{2}{*}{63} & iatrogénie & \multirow{2}{*}{ iatrogénie } & \multirow{2}{*}{$\begin{array}{l}\text { confirme et } \\
\text { élimine }\end{array}$} \\
\hline & MP précoce & & \\
\hline 64 & sd extrapyramidal atypique & autre, ne sait pas & élimine \\
\hline 65 & MP précoce & MP précoce & élimine \\
\hline \multirow{2}{*}{66} & MP avancée & \multirow{2}{*}{ AMS } & \multirow[b]{2}{*}{ confirme } \\
\hline & $\mathrm{DCL}$ & & \\
\hline \multirow{2}{*}{67} & iatrogénie & \multirow{2}{*}{ iatrogénie } & \multirow[b]{2}{*}{ confirme } \\
\hline & psychogène & & \\
\hline 68 & MP précoce & iatrogénie & élimine \\
\hline \multirow{2}{*}{69} & sd extrapyramidal atypique & \multirow{2}{*}{ sd extrapyramidal atypique } & \multirow[b]{2}{*}{ confirme } \\
\hline & AMS & & \\
\hline
\end{tabular}




\begin{tabular}{|c|c|c|c|}
\hline 70 & $\mathrm{DCL}$ & démence vasculaire & élimine \\
\hline \multirow{2}{*}{71} & parkinson vasculaire & \multirow{2}{*}{ iatrogénie } & \multirow[b]{2}{*}{ confirme } \\
\hline & iatrogénie & & \\
\hline 72 & tremblement essentiel & tremblement essentiel & confirme \\
\hline \multirow{2}{*}{73} & tremblement essentiel & \multirow{2}{*}{ MP précoce } & confirme et \\
\hline & MP précoce & & élimine \\
\hline 74 & MP précoce & autre, ne sait pas & élimine \\
\hline \multirow{2}{*}{75} & MP précoce & \multirow{2}{*}{ psychogène } & \multirow[b]{2}{*}{ renforce } \\
\hline & psychogène & & \\
\hline 76 & MP précoce & MP précoce & confirme \\
\hline \multirow{3}{*}{77} & MP avancée & \multirow{3}{*}{ psychogène } & \multirow[b]{3}{*}{ renforce } \\
\hline & parkinson vasculaire & & \\
\hline & psychogène & & \\
\hline 78 & tremblement essentiel & tremblement essentiel & renforce \\
\hline 79 & tremblement essentiel & tremblement essentiel & élimine \\
\hline 80 & PSP & autre, ne sait pas & élimine \\
\hline 81 & DCB & autre, ne sait pas & confusion \\
\hline \multirow{3}{*}{82} & MP avancée & \multirow{3}{*}{ MP avancée } & \multirow[b]{3}{*}{ élimine } \\
\hline & parkinson vasculaire & & \\
\hline & tremblement essentiel & & \\
\hline 83 & tremblement essentiel & tremblement essentiel & confirme \\
\hline \multirow{2}{*}{84} & MP avancée & \multirow{2}{*}{ MP avancée } & \multirow[b]{2}{*}{ confirme } \\
\hline & AMS & & \\
\hline 85 & PSP & DFT & élimine \\
\hline \multirow{2}{*}{86} & MP avancée & \multirow{2}{*}{$\begin{array}{l}\text { origine post-lésionnelle } \\
\text { post-radique }\end{array}$} & \multirow[b]{2}{*}{ confirme } \\
\hline & origine post-lésionnelle post-radique & & \\
\hline 87 & MP précoce & MP précoce & renforce \\
\hline \multirow{2}{*}{88} & iatrogénie & \multirow{2}{*}{ iatrogénie } & \multirow[b]{2}{*}{ confirme } \\
\hline & MP précoce & & \\
\hline
\end{tabular}




\begin{tabular}{|l|l|l|l|}
\hline $\mathbf{8 9}$ & tremblement essentiel & tremblement essentiel & confirme \\
\hline \multirow{2}{*}{90} & sd extrapyramidal atypique & tremblements dystoniques & \multirow{2}{*}{ élimine } \\
\cline { 2 - 4 } & MP précoce & iatrogénie & \\
\hline \multirow{2}{*}{$\mathbf{9 1}$} & MP précoce & & confirme \\
\cline { 2 - 4 } & iatrogénie &
\end{tabular}

Tableau 5 : Hypothèses diagnostiques initiales et diagnostic final retenu pour les 91 cas.

5. Evaluation de l'intérêt de la TEP cérébrale à la 18F-FDOPA par les neurologues

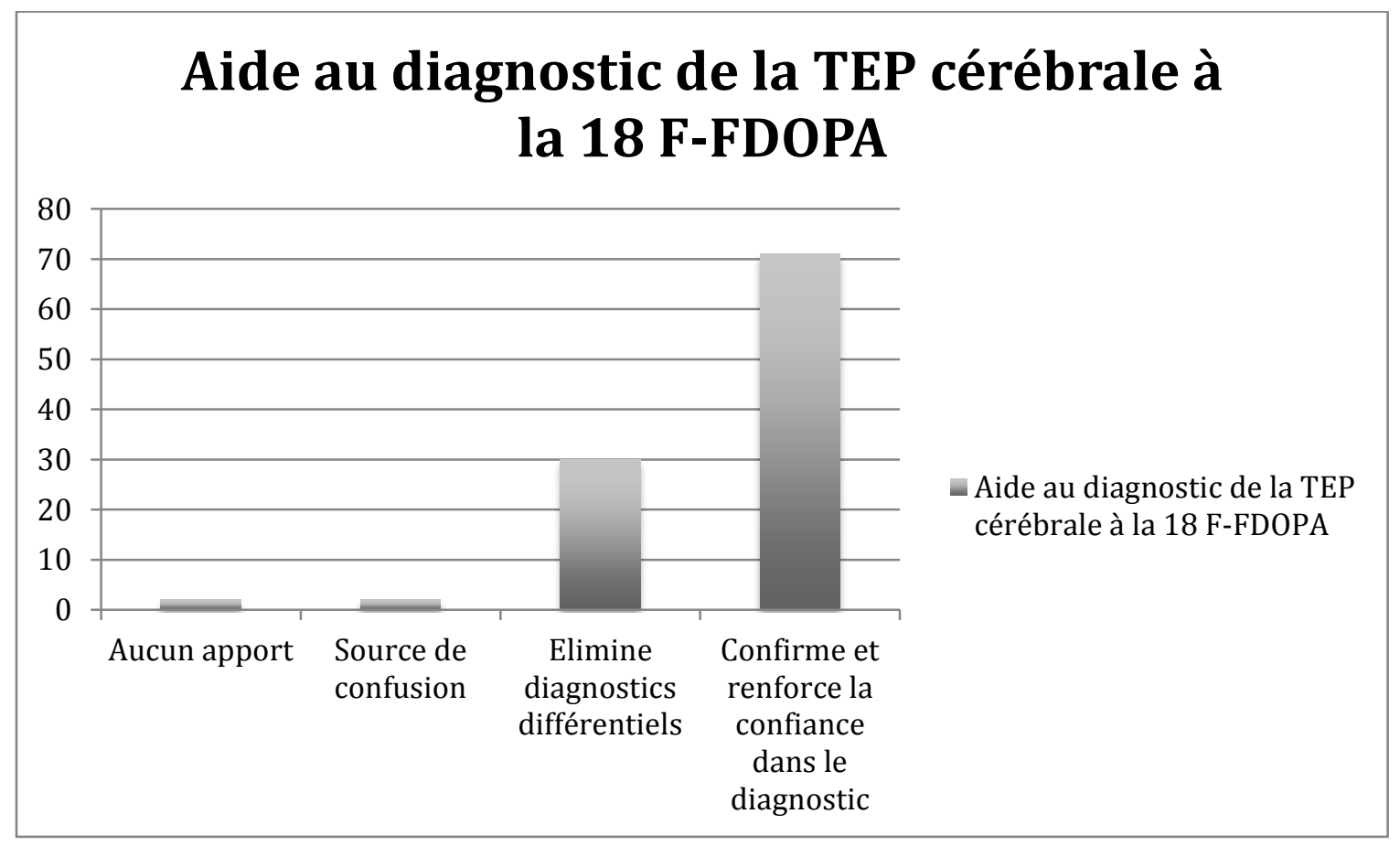

Figure 13 : Aide au diagnostic de la TEP cérébrale à la 18 F-FDOPA

Nous avons demandé aux médecins neurologues, pour chaque examen prescrit, dans quelle mesure le résultat de la TEP les avait aidés pour le diagnostic du patient (figure 13):

- la TEP leur a permis de renforcer leur confiance dans leur diagnostic et de confirmer leur diagnostic dans 71,4\% (65/91) des cas, soit pour plus de 2 examens sur 3.

- la TEP a aussi permis d'éliminer des diagnostics différentiels dans 30\% (27/91) des cas. 
- dans seulement 2,2\% des cas la TEP ne leur apportait rien ou était source de confusion.

Parmi les 71 patients chez qui les neurologues ont trouvé une cohérence entre le résultat de la TEP et leur examen clinique ; l'examen a renforcé leur confiance dans leur diagnostic et a confirmé leur diagnostic pour 84,5\% (60/71) des patients.

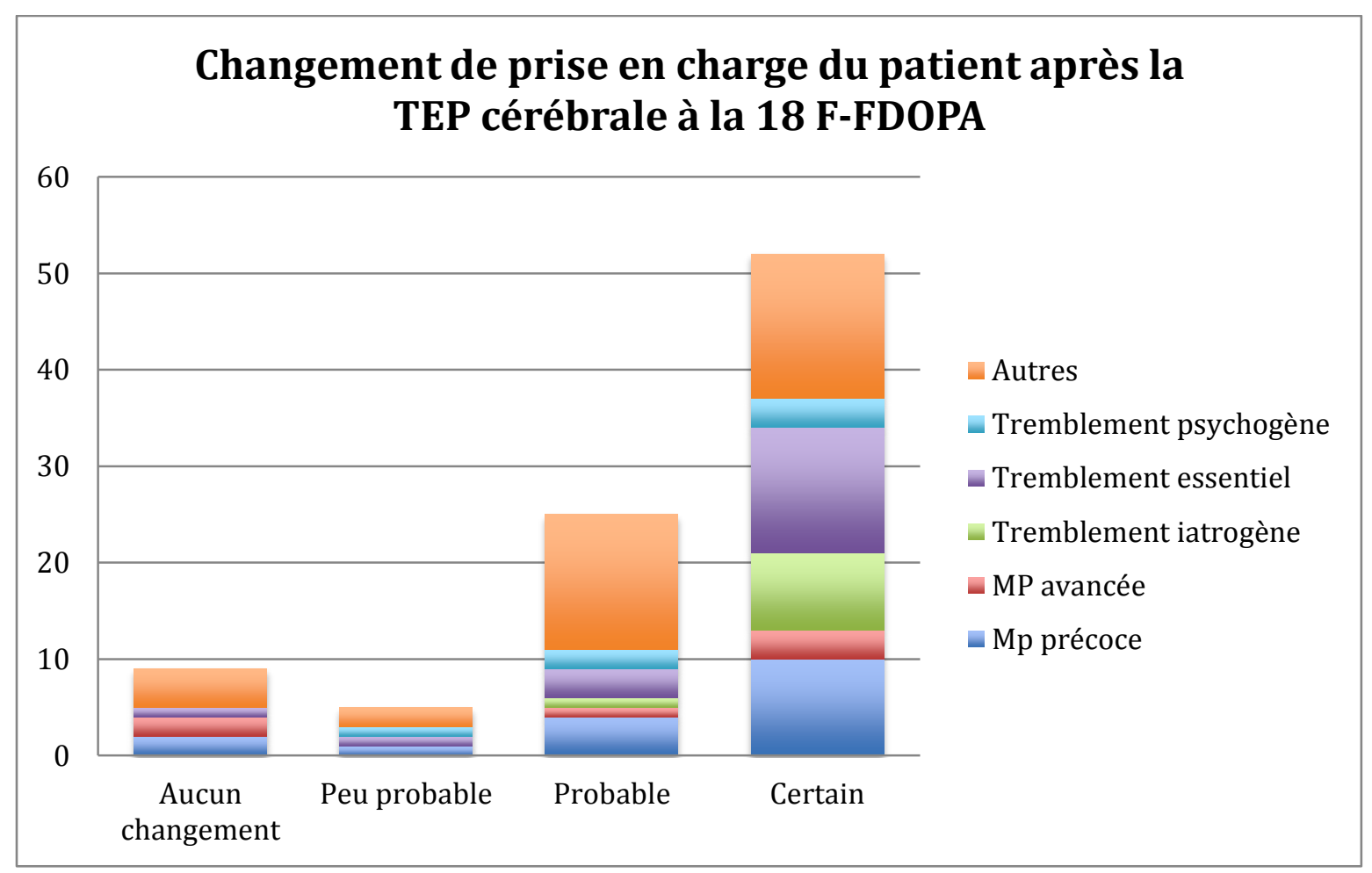

Figure 14 : Evaluation du changement de prise en charge du patient après la TEP cérébrale à la $18 \mathrm{~F}$ FDOPA.

Les changements de prise en charge induits par les résultats de la TEP à la $18 \mathrm{~F}$ FDOPA sont décrits figure 14:

- dans 57\% $(n=52)$ des cas la TEP a induit de façon certaine un changement de prise en charge du patient,

- elle induira probablement un changement dans 27,5\% (n=25) des cas,

- pour seulement $10 \%(n=9)$ des patients, elle n'a conduit à aucun changement dans la prise en charge du patient. 
Ce changement de prise en charge était certain dans près de 2 cas sur 3 pour les MP précoces $(n=10 / 17)$, dans 1 cas sur 2 dans les MP avancées $(n=3 / 6)$, dans 8 cas sur 9 dans les tremblements d'origine iatrogène $(n=8 / 9)$ et dans près de 3 cas sur 4 dans les tremblements essentiels $(n=13 / 18)$.

Les différents types de changement de prise en charge sont exposés figure 15. Lorsqu'il y avait un changement de prise en charge $(n=82 / 91)$ le plus important était l'arrêt ou le début d'un traitement dans $73 \%$ des cas $(n=60 / 82)$ venait ensuite la modification du suivi dans $22 \%$ des cas $(n=18 / 82)$.

Il y avait un changement de traitement pour $84 \%$ des MP $(n=16 / 19), 62,5 \%$ des autres syndromes extrapyramidaux $(n=10 / 16)$ et pour $72 \%$ des patients $(n=34 / 47)$ chez qui il n'était pas retenu d'atteinte dopaminergique (c'est à dire les tremblements essentiels, psychogènes, iatrogènes ou normaux).

Dans la grande majorité des cas $(84 \%$; n=69/82) il n'y avait pas de prescription d'autre examen complémentaire en particulier lorsque le diagnostic retenu était une MP ou un tremblement d'origine essentielle ou psychogène.

Il y a eu une demande d'affection longue durée pour 5 MP précoce soit $6 \%$ des patients $(n=5 / 82)$.

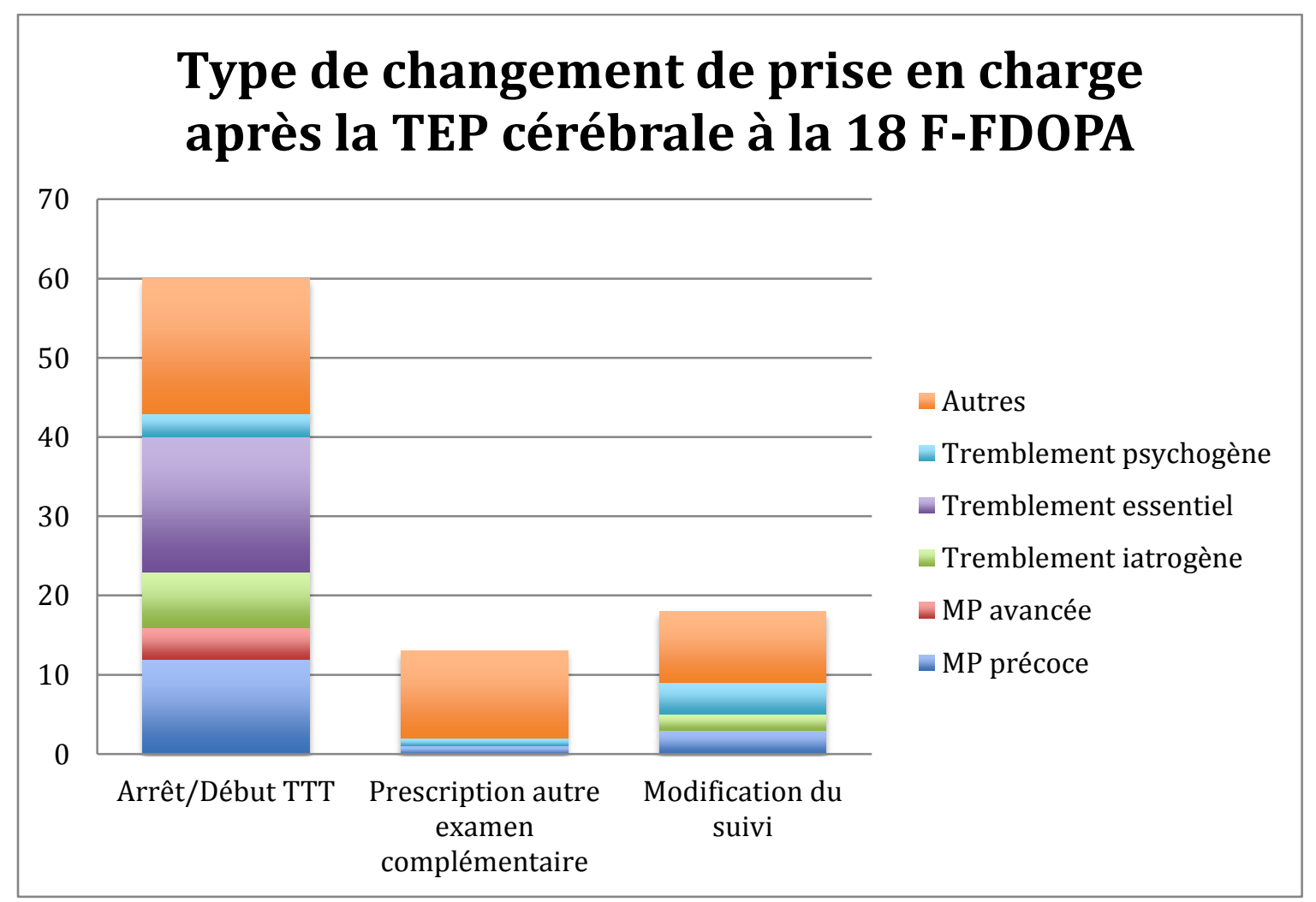

Figure 15 : Type de changement de prise en charge après la TEP cérébrale à la 18 F-FDOPA 
- Parmi les examens normaux (n=49), 69\% des examens (34/49) l'étaient lorsque l'atteinte de la voie dopaminergique avant la TEP était improbable ou peu probable selon le neurologue.

- Parmi les examens anormaux (n=42), 81\% (34/42) l'étaient lorsque l'atteinte de la voie dopaminergique avant la TEP était probable ou très probable.

Pour les patients $(n=15)$ chez qui l'atteinte de la voie dopaminergique avant la TEP était très probable selon le neurologue, $73 \%$ ont eu comme diagnostic final retenu une MP précoce ou avancée (11/15), 20\% un autre syndrome extra-pyramidal (3/15) et un seul patient a été retenu comme normal, sans atteinte dopaminergique. Pour $10 \%$ des patients, le médecin a prescrit de nouveau l'examen pour suivre l'évolution.

Résumé des points essentiels:

L'examen clinique du neurologue était cohérent avec le résultat de la TEP dans $\mathbf{7 8 \%}$ des cas $(n=71 / 91)$.

La TEP a permis de changer le diagnostic retenu :

dans 1/3 des cas lorsqu'une MP était suspectée,

dans 1 cas sur 2 lorsqu'un autre syndrome extra-pyramidal était suspecté.

Selon les neurologues, la TEP a renforcé leur confiance dans leur diagnostic et a confirmé leur diagnostic dans $\mathbf{7 1 , 4 \%}$ des cas.

Dans 57\% des cas la TEP a induit de façon certaine un changement de prise en charge du patient.

La TEP a induit un changement de traitement (début ou arrêt) dans $\mathbf{7 3 \%}$ des cas.

\section{B. RÉSULTATS DE L'ANALYSE VISUELLE}

L'analyse visuelle est la méthode de routine utilisée dans notre service de médecine nucléaire qui consiste à rechercher une hypofixation striatale en général plus marquée au niveau du putamen postérieur et s'étendant au noyau caudé. Nous allons dans ce paragraphe confronter nos résultats de l'analyse visuelle au 
diagnostic final retenu par le neurologue afin de déterminer la sensibilité et la spécificité de cette méthode dans notre étude.

Les médecins nucléaires remplissaient la fiche d'interprétation (annexe 2) concernant l'évaluation visuelle, ces derniers avaient le choix entre 4 items :

- $\quad$ Type 1 : normal

- Type 2 : fixation asymétrique

- $\quad$ Type 3 : fixation caudée exclusive

- Type 4 : fixation caudée exclusive très faible avec bruit de fond très élevé.

La figure 7 (Partie Mise au point, chapitre D, paragraphe 1) donne un exemple d'images illustrant ces 4 types de fixation.

Nous avons obtenu 58 examens de type 1 (soit 63,7\%), 18 examens de type 2 (soit 19,8\%), 13 examens de type 3 (soit 14,3\%) et 2 examens de type 4 (soit 2,2\%) soit 33 examens classés dans les anormaux en analyse visuelle.

9 patients ayant un examen de type 1 normal ont été retenus comme malades par le diagnostic final du neurologue. Les maladies retenues étaient: maladie de parkinson, dégénérescence cortico-basale, parkinson vasculaire et syndrome pyramidal atypique. Un patient pour lequel l'examen avait une analyse visuelle de type 2 a été retenu comme sans atteinte dopaminergique dans le diagnostic neurologique final.

La sensibilité de l'analyse visuelle est de 78\% et la spécificité de 98\%.

\section{RÉSULTATS DE L'ANALYSE SEMI-QUANTITATIVE}

L'interprétation de la TEP cérébrale à la 18 F-FDOPA par le médecin nucléaire repose actuellement sur l'analyse visuelle. Afin de limiter les biais inter mais aussi intra-observateur il est possible de réaliser une analyse semi-quantitative des images à l'aide d'outils de mesure de fixation. Les 91 examens TEP à la 18F-FDOPA ont fait secondairement l'objet d'un travail de semi-quantification afin d'établir des seuils diagnostiques qui pourront être utilisés par la suite en routine clinique. 


\section{Les patients étudiés pour l'analyse semi-quantitative}

Parmi les 91 patients ayant bénéficié d'une TEP cérébrale à la 18F-FDOPA (figure 16), nous avons pour l'analyse semi-quantitative classé les patients en deux catégories selon le diagnostic final retenu par le neurologue:

- les patients dits « normaux » c'est-à-dire les patients n'ayant pas de maladie de parkinson, ni de syndrome parkinsonien atypique. Cela inclut également les patients n'ayant pas d'atteinte dopaminergique mais pouvant être diagnostiqués comme tremblement essentiel, ou d'origine iatrogène ou psychogène.

- les patients dits « anormaux » c'est-à-dire les patients diagnostiqués maladie de parkinson ou un autre syndrome parkinsonien présentant une atteinte dopaminergique.

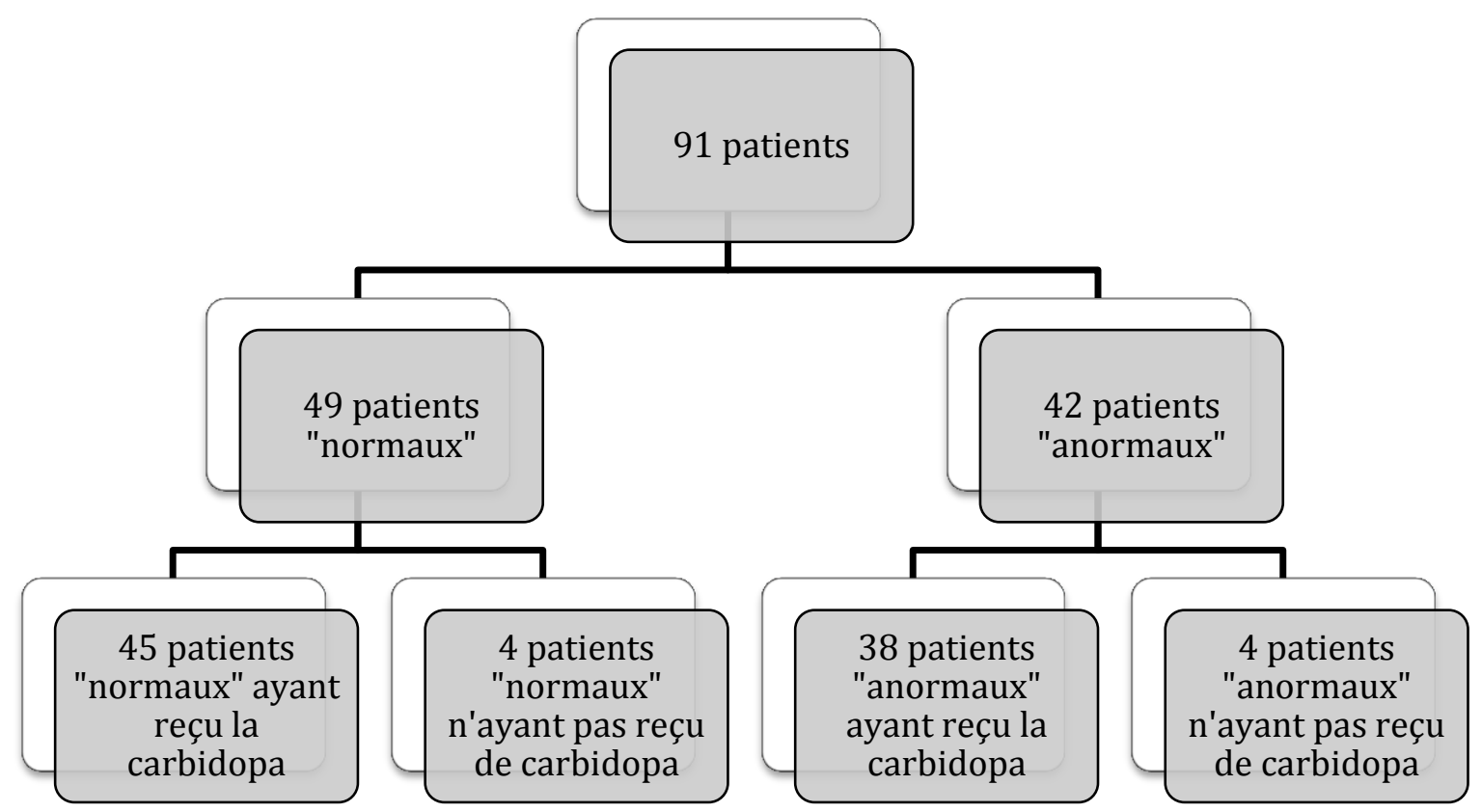

Figure 16 : Patients inclus dans l'étude

Nous avons obtenu 49 patients dits «normaux », parmi eux 45 patients ont reçu de la Carbidopa avant l'examen, 4 patients n'en ont pas reçu en général par contre-indication cardiaque. 
Nous avons obtenu 42 patients dans le groupe dit des patients « anormaux » dont 4 patients n'ayant pas reçu de Carbidopa également pour contre-indication cardiaque.

\section{Etude de reproductibilité inter-observateur}

Avant d'analyser les résultats semi-quantitatifs et afin d'évaluer la reproductibilité inter-observateur, 30 examens tirés au sort de façon aléatoire parmi les 91 examens tous confondus ont été analysés de façon semi-quantitative par un observateur différent.

La reproductibilité était excellente avec des coefficients de corrélation intra-classe proche de 1 (tableau 6) pour l'ensemble des outils de mesures $(\mathrm{p}<0,0001)$.

\begin{tabular}{|c|c|c|c|c|}
\hline \multirow[t]{2}{*}{ Mesures des SUV } & \multirow{2}{*}{$\begin{array}{l}\text { Coefficient de } \\
\text { corrélation } \\
\text { intra-classe }\end{array}$} & \multirow{2}{*}{$\begin{array}{l}\text { Significativité } \\
\text { (p) }\end{array}$} & \multicolumn{2}{|c|}{ Intervalle de confiances à 95\% } \\
\hline & & & Borne inférieure & Borne supérieure \\
\hline SUV occipital & 0,870 & $<0,0001$ & 0,746 & 0,936 \\
\hline SUV striatum droit & 1 & $<0,0001$ & 1 & 1 \\
\hline $\begin{array}{ll}\text { SUV } & \text { striatum } \\
\text { gauche } & \end{array}$ & 0,997 & $<0,0001$ & 0,994 & 0,999 \\
\hline $\begin{array}{l}\text { SUV noyau caudé } \\
\text { droit }\end{array}$ & 0,939 & $<0,0001$ & 0,876 & 0,970 \\
\hline $\begin{array}{l}\text { SUV noyau caudé } \\
\text { gauche }\end{array}$ & 0,983 & $<0,0001$ & 0,965 & 0,992 \\
\hline $\begin{array}{l}\text { SUV putamen } \\
\text { antérieur droit }\end{array}$ & 1 & $<0,0001$ & 1 & 1 \\
\hline $\begin{array}{l}\text { SUV putamen } \\
\text { antérieur gauche }\end{array}$ & 0,996 & $<0,0001$ & 0,991 & 0,998 \\
\hline $\begin{array}{l}\text { SUV putamen } \\
\text { postérieur droit }\end{array}$ & 0,956 & $<0,0001$ & 0,910 & 0,979 \\
\hline $\begin{array}{l}\text { SUV putamen } \\
\text { postérieur gauche }\end{array}$ & 0,971 & $<0,0001$ & 0,941 & 0,986 \\
\hline
\end{tabular}

Tableau 6 : Relecture : coefficients de corrélation intra-classe et intervalle de confiance pour l'ensemble des outils de mesure. 


\section{Etudes préliminaires à l’analyse semi-quantitative}

Afin de s'assurer de la validité de nos mesures nous avons d'abord testé les valeurs semi-quantitatives (SUV des différents VOI) chez les sujets normaux afin de s'assurer de la présence ou non d'une différence significative des valeurs selon la prise de Carbidopa, ainsi que selon le côté droit ou gauche du cerveau, l'âge et le sexe.

Nous avons comparé les valeurs des mesures des SUV chez les patients ayant reçu de la Carbidopa $(n=45)$ de ceux n'en ayant pas reçu $(n=4)$. Toutes les mesures étaient visuellement inférieures chez les patientes n'ayant pas reçu de Carbidopa (Figure 17) et nous avons trouvé une différence significative $(p=0,047)$ au niveau des mesures réalisées sur le putamen antérieur. Pour les autres valeurs la différence était à la limite de la significativité avec des p proches de 0,05. Ce manque de significativité pouvait être expliqué par la différence d'effectif entre les deux groupes. Nous avons donc choisi d'exclure les patients n'ayant pas reçu de Carbidopa pour la suite de l'analyse portant sur les SUV. Par contre sur les rapports de fixation (SOR, COR, POR ant et POR post) comme on pouvait s'y attendre il n'y avait pas de différence significative des données avec ou sans Carbidopa et nous avons donc choisi de garder l'effectif complet de patients pour l'analyse des rapports de fixation afin de ne pas perdre de puissance.

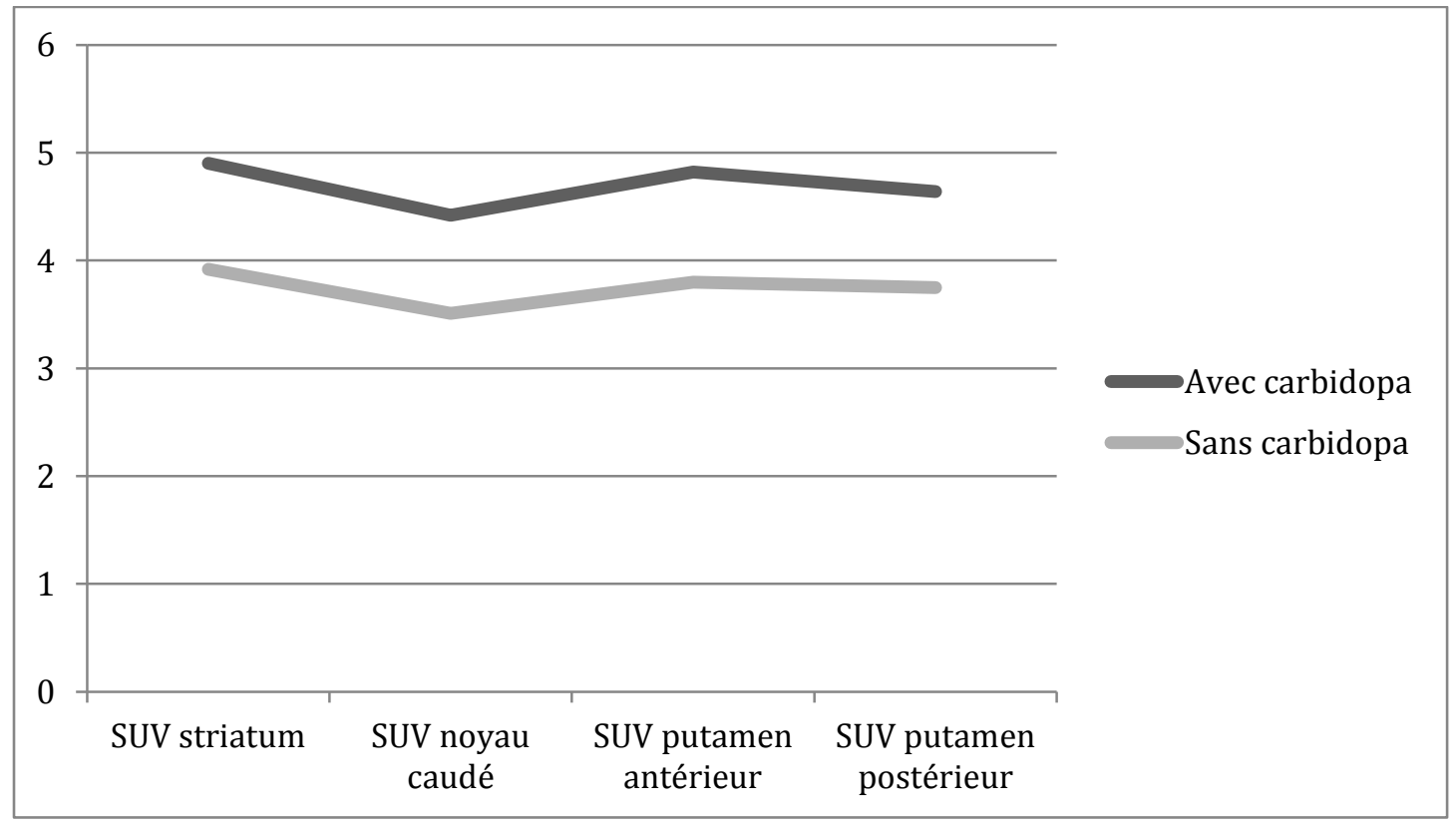

Figure 17: Comparaison des valeurs de SUV avec et sans prise de Carbidopa 
Il n'y avait pas de différence significative selon le test pour échantillons appareillés entre les valeurs mesurées du côté droit et les valeurs mesurées à gauche.

La population de sujets normaux avait un âge médian de 73,7 ans, l'âge minimum était de 50 ans et le maximum de 86 ans. 22 patients avaient moins de 73,7 ans et 23 patients avaient plus de 73,7 ans. Nous n'avons pas trouvé de différence significative entre les valeurs des mesures chez les patients de plus de 73,7 ans et ceux de moins de 73,7 ans. Cela signifie qu'il n'y avait pas de corrélation inverse entre le SUV et l'âge. Cette absence d'effet âge pour la TEP à la 18F-FDOPA était en accord avec les données de la littérature.

La population des sujets dits «normaux» comprenait 24 femmes et 21 hommes, et nous n'avons pas trouvé de différence significative des valeurs entre les deux sexes.

\section{Analyse des données}

\subsection{Valeurs semi-quantitatives}

\begin{tabular}{|l|l|l|l|l|l|l|l|}
\hline & \multicolumn{2}{|l|}{ Patients dits «normaux » } & & \multicolumn{2}{l|}{ Patients dits « anormaux » } \\
\hline & $\mathrm{n}$ & Moyenne & Ecart type & $\mathrm{p}$ & $\mathrm{n}$ & Moyenne & Ecart type \\
\hline SUV striatum & 45 & 4,90 & 0,95 & 0,003 & 38 & 4,27 & 0,95 \\
\hline $\begin{array}{l}\text { SUV noyau } \\
\text { caudé }\end{array}$ & 45 & 4,42 & 0,89 & 0,272 & 38 & 4,20 & 0,94 \\
\hline $\begin{array}{l}\text { SUV putamen } \\
\text { antérieur }\end{array}$ & 45 & 4,82 & 0,95 & $<0,001$ & 38 & 3,99 & 0,97 \\
\hline $\begin{array}{l}\text { SUV putamen } \\
\text { postérieur }\end{array}$ & 45 & 4,64 & 0,98 & $<0,001$ & 38 & 3,39 & 0,95 \\
\hline SOR & 45 & 3,04 & 0,80 & $<0,001$ & 38 & 2,61 & 0,38 \\
\hline COR & 45 & 2,74 & 0,71 & 0,022 & 38 & 2,56 & 0,35 \\
\hline POR ant & 45 & 2,99 & 0,86 & $<0,001$ & 38 & 2,43 & 0,47 \\
\hline POR post & 45 & 2,88 & 0,86 & $<0,001$ & 38 & 2,073 & 0,51 \\
\hline
\end{tabular}

Tableau 7 : Moyenne des SUV et des rapports de fixation (SOR, COR, POR antérieur et postérieur) 
Nous avons exposé dans le tableau 7, pour chacun des deux groupes de patients, les valeurs moyennes droites/gauches des SUV du striatum, noyau caudé, putamen antérieur, postérieur ainsi que les valeurs moyennes des rapports de fixation SOR, COR, POR antérieur et POR postérieur. Il est intéressant de noter que la seule valeur non significativement différente entre les patients normaux et anormaux est le SUV moyen du noyau caudé.

Nous avons distingué les valeurs des SUV et des rapports de fixation (SOR, COR, POR ant et POR post) chez les patients anormaux non pas en droite/gauche mais en côté controlatéral à l'atteinte motrice prédominante et côté homolatéral à l'atteinte motrice prédominante selon les indications données par le médecin neurologue dans le questionnaire (item : latéralisation de l'atteinte motrice). Les valeurs moyennes sont exposées dans le tableau 8.

\begin{tabular}{|c|c|c|c|c|c|c|}
\hline & \multicolumn{6}{|c|}{ Moyenne des rapports de fixation chez les patients « anormaux » } \\
\hline & \multirow[t]{2}{*}{$\mathrm{n}$} & \multicolumn{2}{|c|}{$\begin{array}{l}\text { Côté controlatéral à l'atteinte } \\
\text { motrice prédominante }\end{array}$} & \multicolumn{2}{|c|}{$\begin{array}{l}\text { Côté homolatéral à } \\
\text { l'atteinte motrice } \\
\text { prédominante }\end{array}$} & \multirow[t]{2}{*}{$\mathrm{p}$} \\
\hline & & Moyenne & Ecart type & Moyenne & Ecart type & \\
\hline SOR & 38 & 2,57 & 0,38 & 2,64 & 0,40 & 0,004 \\
\hline COR & 38 & 2,53 & 0,35 & 2,6 & 0,37 & 0,15 \\
\hline POR ant & 38 & 2,37 & 0,47 & 2,51 & 0,47 & 0,001 \\
\hline POR post & 38 & 2 & 0,51 & 2,15 & 0,49 & 0,005 \\
\hline $\begin{array}{l}\text { SUV } \\
\text { striatum }\end{array}$ & 38 & 4,23 & 0,96 & 4,31 & 0,97 & 0,89 \\
\hline $\begin{array}{l}\text { SUV noy } \\
\text { caudé }\end{array}$ & 38 & 4,14 & 0,95 & 4,26 & 0,95 & 0,006 \\
\hline SUV put ant & 38 & 3,88 & 0,99 & 4,31 & 0,97 & $<0,001$ \\
\hline $\begin{array}{l}\text { SUV put } \\
\text { post }\end{array}$ & 38 & 3,32 & 1,03 & 3,46 & 0,94 & 0,119 \\
\hline
\end{tabular}

Tableau 8: SUV et rapports de fixation moyen chez les patients anormaux $(\mathrm{n}=38)$ selon le côté de l'atteinte motrice. 
Les valeurs des rapports de fixation du côté controlatéral à l'atteinte motrice prédominante étaient significativement inférieures à celles du côté homolatéral sur les quatre outils de mesure concernant les rapports : SOR, COR, POR ant et POR post ainsi que sur les SUV du noyau caudé, des putamens antérieur et postérieur.

Les boites à moustaches suivantes montrent la répartition des valeurs des SUV (figure 18) et des rapports de fixation (figure 19) sur l'ensemble des régions d'intérêt selon le côté homo ou controlatéral à l'atteinte motrice prédominante. 


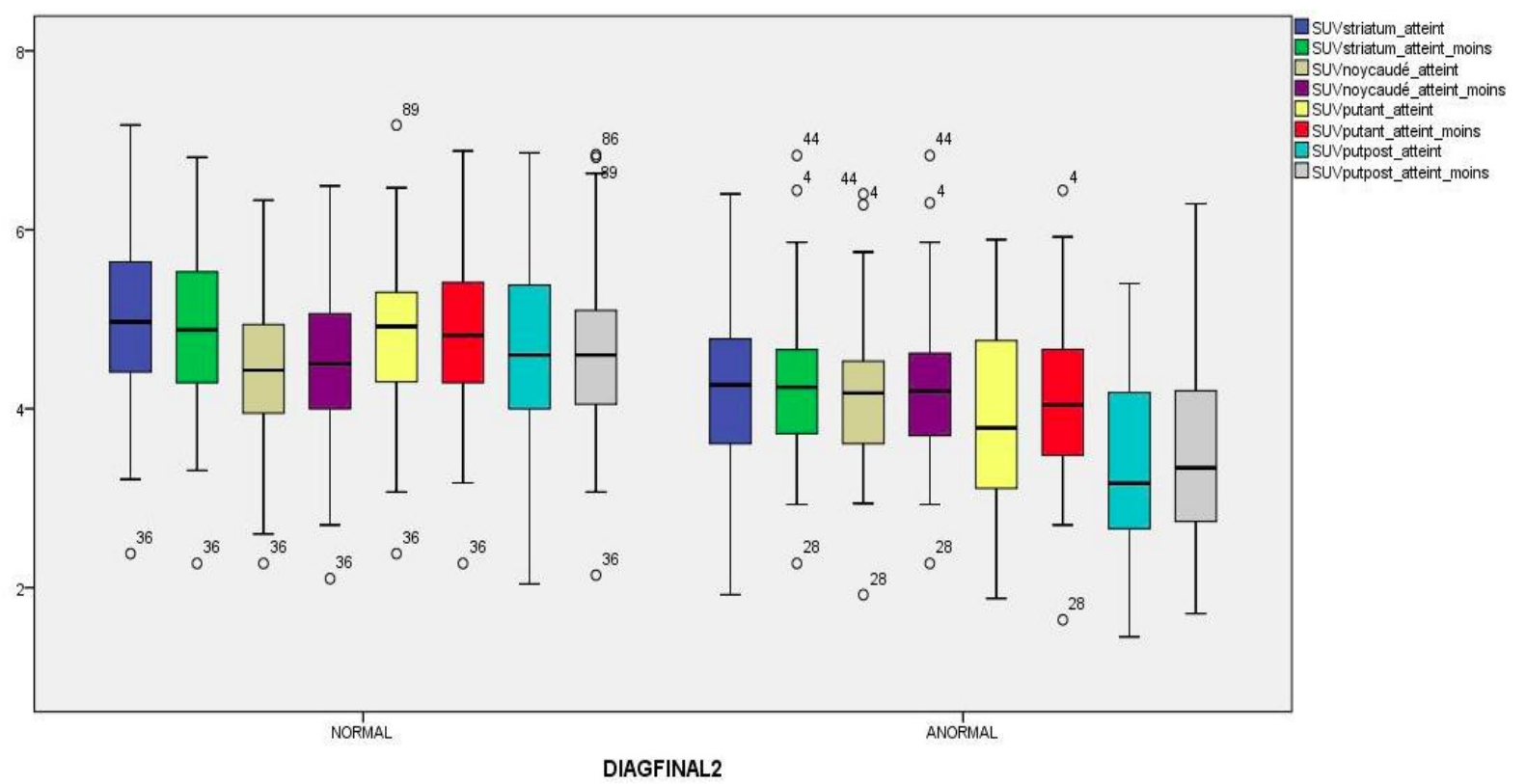

Figure 18: Boite à moustache SUV controlatéraux (atteint) et homolatéraux (moins atteint) à l'atteinte motrice prédominante chez les patients normaux et anormaux.

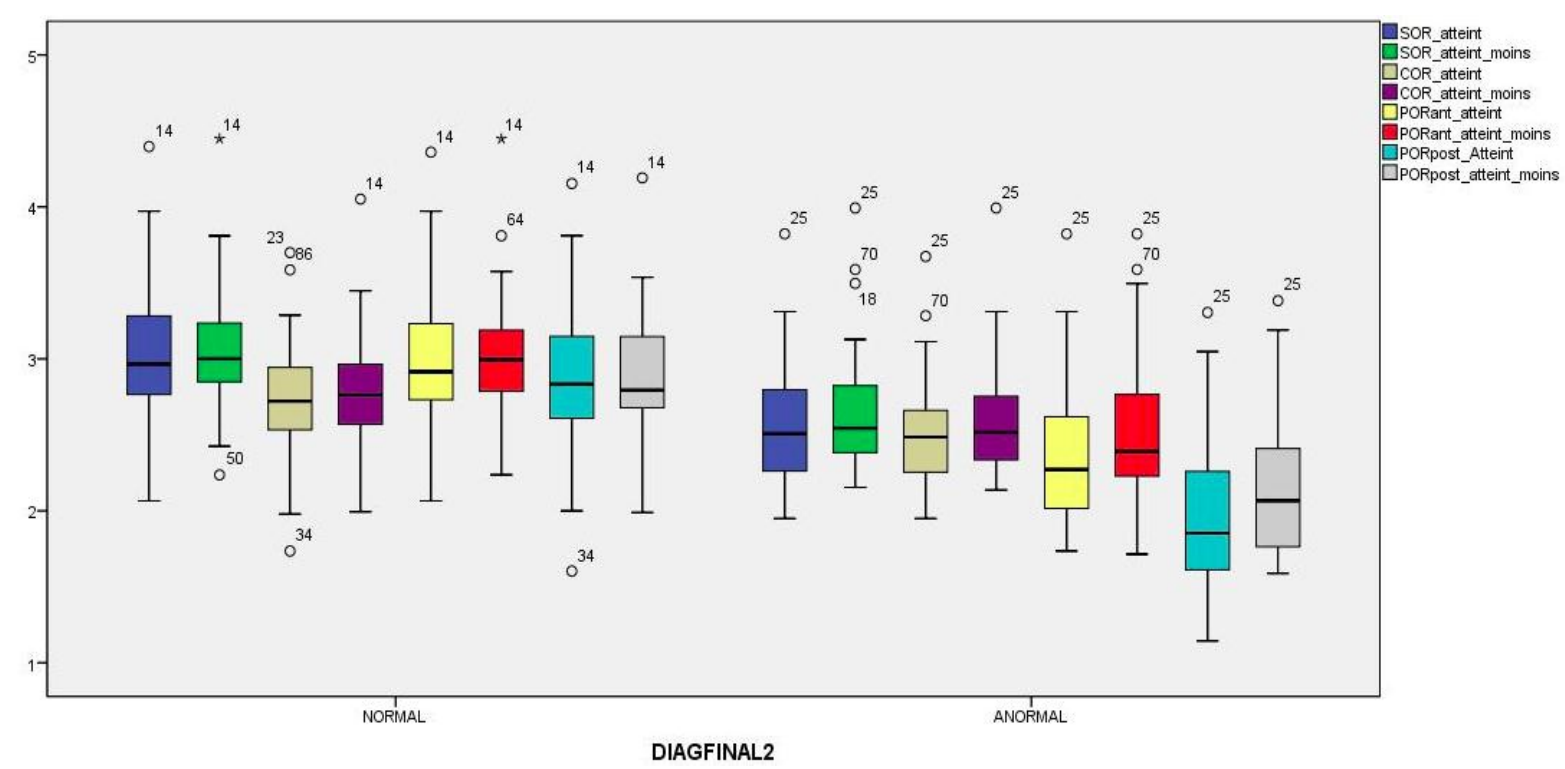

Figure 19: Boite à moustache des rapports de fixation controlatéraux (atteint) et homolatéraux (moins atteint) à l'atteinte motrice prédominante chez les patients normaux et anormaux. 


\subsection{Courbes ROC : figures de mérite SUV controlatéraux et homolatéraux à l'atteinte}

motrice prédominante

Les courbes ROC ont été établies sur les valeurs des SUV mesurées par des VOI au niveau du striatum, du noyau caudé, du putamen antérieur et du putamen postérieur premièrement du côté controlatéral à l'atteinte motrice prédominante et secondairement du côté homolatéral.

4.2.1 Courbes ROC : SUV du côté controlatéral à l'atteinte motrice prédominante.

La figure 20 expose les courbes ROC établies sur les valeurs des SUV du striatum, du noyau caudé, du putamen antérieur et postérieur du côté controlatéral à l'atteinte motrice prédominante.

Selon l'indice de Youden (tableau 7) nous avons obtenu pour chaque figure de mérite une valeur de sensibilité et de spécificité minimisant le nombre de faux positif et le nombre de faux négatif (selon le point sur la courbe le plus proche de 1).

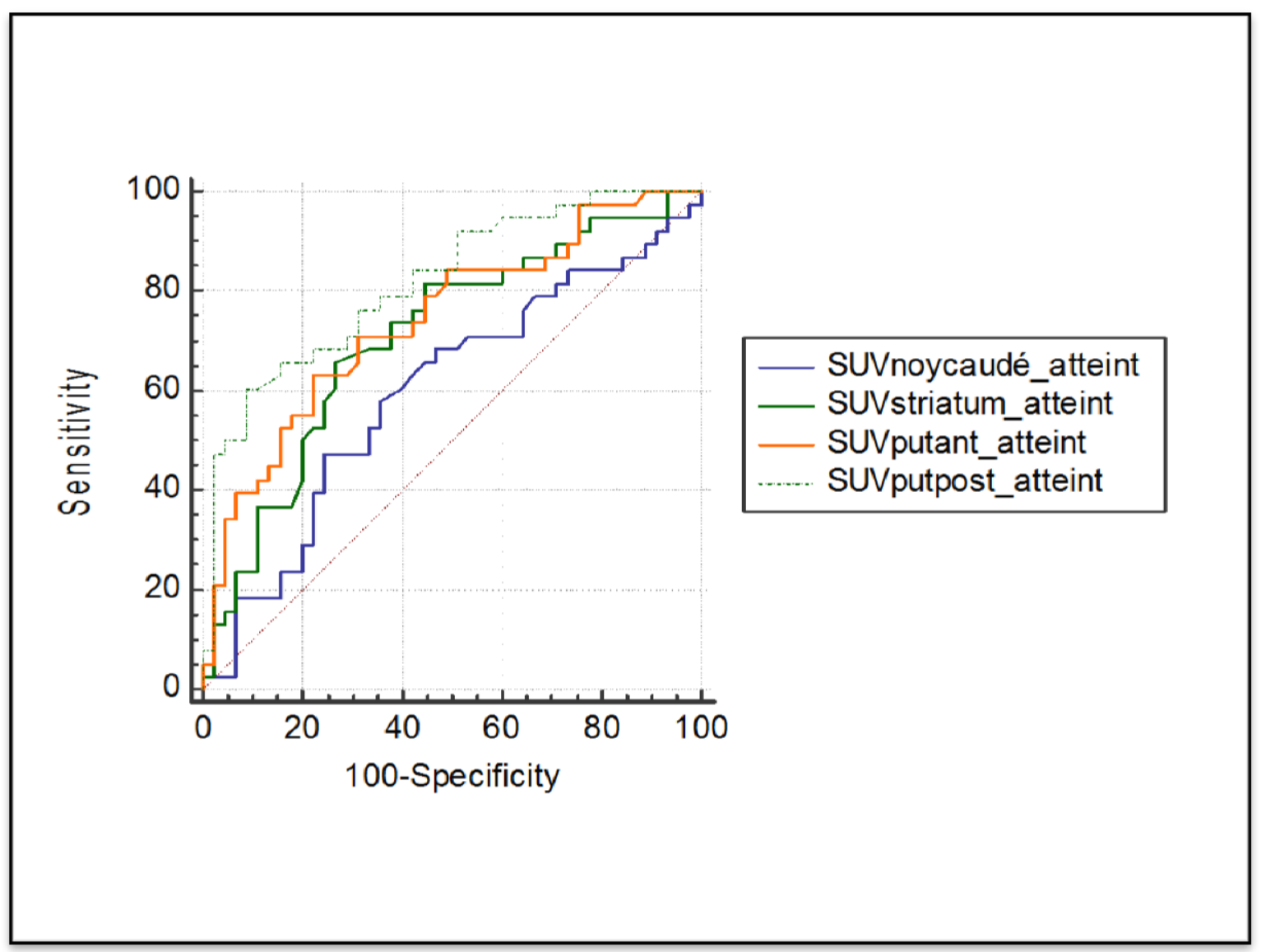

Figure 20 : Courbes ROC des SUV controlatéraux à l'atteinte motrice prédominante. 
La courbe obtenant la plus grande aire sous la courbe $(0,817)$ est celle du SUV du putamen postérieur avec une sensibilité de $60,5 \%$ et une spécificité de 91,1\%, venait ensuite la courbe du SUV du putamen antérieur $(0,742)$ puis celle du SUV du striatum $(0,708)$ et enfin celle du SUV du noyau caudé $(0,598)$. Les différents paramètres diagnostics des 4 courbes ROC ainsi que l'indice de Youden sont exposés dans le tableau 9.

\begin{tabular}{|c|c|c|c|c|c|c|}
\hline $\begin{array}{l}\text { Rapport de } \\
\text { fixation }\end{array}$ & $\begin{array}{l}\text { Valeur } \\
\text { seuil }\end{array}$ & Sensibilité & IC 95\% & Spécificité & IC 95\% & $\begin{array}{ll}\text { Index } & \text { de } \\
\text { Youden } & \end{array}$ \\
\hline $\begin{array}{l}\text { SUV } \\
\text { striatum }\end{array}$ & $\leq 4,52$ & 65,79 & $48,6-80,4$ & 73,33 & $58,1-85,4$ & 0,3912 \\
\hline $\begin{array}{l}\text { SUV noyau } \\
\text { caudé }\end{array}$ & $\leq 3,94$ & 47,37 & $31,0-64,2$ & 75,56 & $\begin{array}{ll}60,5 & - \\
87,1 & \end{array}$ & 0,2292 \\
\hline $\begin{array}{l}\text { SUV } \\
\text { putamen } \\
\text { antérieur }\end{array}$ & $\leq 4,02$ & 63,16 & $46,0-78,2$ & 77,78 & $62,9-88,8$ & 0,4094 \\
\hline $\begin{array}{l}\text { SUV } \\
\text { putamen } \\
\text { postérieur }\end{array}$ & $\leq 3,37$ & 60,53 & $43,4-76$ & 91,11 & $\begin{array}{ll}78,8 & - \\
97,5 & \end{array}$ & 0,5164 \\
\hline
\end{tabular}

Tableau 9: Sensibilité et spécificité des différentes figures de mérite du côté controlatéral à l'atteinte motrice prédominante

Le tableau 10 expose les différentes aires sous la courbe ainsi que leur intervalle de confiance à 95\%.

\begin{tabular}{|l|l|l|}
\hline Variable & AUC & $95 \%$ CI \\
\hline SUV striatum controlatéral & 0,708 & $0,598-0,803$ \\
\hline $\begin{array}{l}\text { SUV noyau caudé } \\
\text { controlatéral }\end{array}$ & 0,598 & $0,485-0,704$ \\
\hline $\begin{array}{l}\text { SUV putamen antérieur } \\
\text { controlatéral }\end{array}$ & 0,742 & $0,634-0,832$ \\
\hline $\begin{array}{l}\text { SUV putamen postérieur } \\
\text { controlatéral }\end{array}$ & 0,817 & $0,717-0,893$ \\
\hline
\end{tabular}

Tableau 10 : Aires sous la courbe des courbes ROC controlatérales à l'atteinte motrice. 
La comparaison des aires sous la courbe des courbes ROC entre elles montrait que :

- l'aire sous la courbe du SUV putamen postérieur était significativement supérieure à celle du SUV putamen antérieur ( $\mathrm{p}=0,0043)$, à celle du SUV striatum $(\mathrm{p}=0,0001)$ ainsi qu'à celle du SUV noyau caudé $(\mathrm{p}<0,0001)$;

- l'aire sous la courbe du SUV putamen antérieur était significativement supérieur à celle du SUV striatum $(\mathrm{p}=0,0302)$ ainsi qu'à celle du SUV noyau caudé $(\mathrm{p}<0,0001)$

- l'aire sous la courbe du SUV striatum était significativement supérieure à celle du SUV noyau caudé $(\mathrm{p}<0,0001)$.

\subsubsection{Courbes ROC : SUV du côté homolatéral à l'atteinte motrice prédominante.}

La figure 21 expose les courbes ROC établies sur les valeurs des SUV du striatum, du noyau caudé, du putamen antérieur et postérieur du côté homolatéral à l'atteinte motrice prédominante.

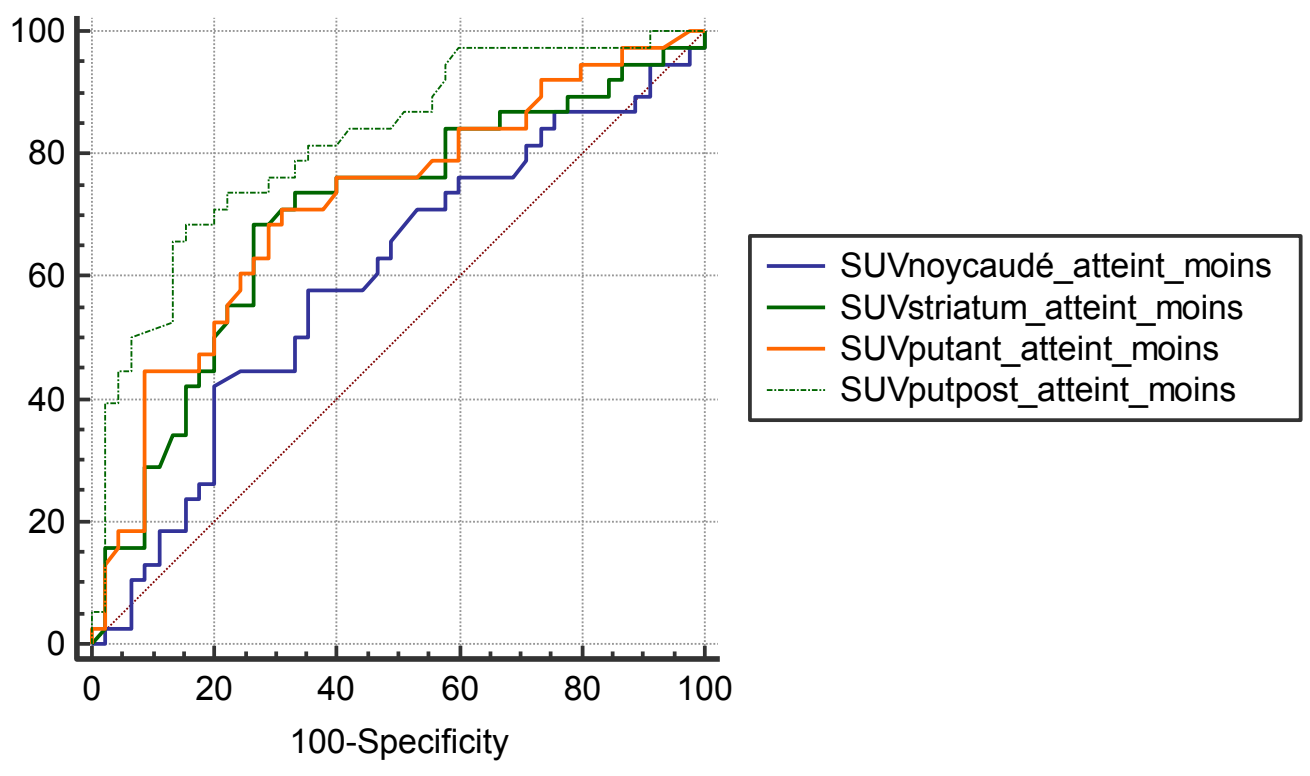

Figure 21 : Courbes ROC des SUV homolatéraux à l'atteinte motrice prédominante. 
La courbe obtenant la plus grande aire sous la courbe $(0,817)$ est aussi celle du SUV du putamen postérieur avec une sensibilité de 68,42\% et une spécificité de $84,4 \%$, venait ensuite la courbe du SUV du putamen antérieur $(0,715)$ puis celle du SUV du striatum $(0,698)$ et enfin celle du SUV du noyau caudé $(0,595)$. Les différents paramètres diagnostics des 4 courbes ROC ainsi que l'indice de Youden sont exposés dans le tableau 11.

\begin{tabular}{|l|l|l|l|l|l|l|}
\hline $\begin{array}{l}\text { Rapport de } \\
\text { fixation }\end{array}$ & Valeur seuil & Sensibilité & IC 95\% & Spécificité & IC 95\% & $\begin{array}{l}\text { Index de } \\
\text { Youden }\end{array}$ \\
\hline $\begin{array}{l}\text { SUV } \\
\text { striatum }\end{array}$ & $\leq 4,51$ & 68,42 & $51,3-82,5$ & 73,33 & $58,1-85,4$ & 0,4175 \\
\hline $\begin{array}{l}\text { SUV noyau } \\
\text { caudé }\end{array}$ & $\leq 4,25$ & 57,89 & $40,8-73,7$ & 64,44 & $48,8-78,1$ & 0,2234 \\
\hline $\begin{array}{l}\text { SUV } \\
\text { putamen } \\
\text { antérieur }\end{array}$ & $\leq 4,54$ & 71,05 & $54,1-84,6$ & 68,89 & $53,4-81,8$ & 0,3994 \\
\hline $\begin{array}{l}\text { SUV } \\
\text { putamen } \\
\text { postérieur }\end{array}$ & $\leq 3,7$ & 68,42 & $51,3-82,5$ & 84,44 & $70,5-93,5$ & 0,5287 \\
\hline
\end{tabular}

Tableau 11 Sensibilité et spécificité des différentes figures de mérite du côté homolatéral à l'atteinte motrice prédominante

Le tableau 12 expose les différentes aires sous la courbe ainsi que leur intervalle de confiance à 95\%.

\begin{tabular}{|l|l|l|}
\hline Variable & AUC & $95 \%$ CI \\
\hline SUV striatum homolatéral & 0,698 & $0,587-0,794$ \\
\hline SUV noyau caudé homolatéral & 0,595 & $0,482-0,702$ \\
\hline $\begin{array}{l}\text { SUV putamen antérieur } \\
\text { homolatéral }\end{array}$ & 0,715 & $0,606-0,809$ \\
\hline $\begin{array}{l}\text { SUV putamen postérieur } \\
\text { homolatéral }\end{array}$ & 0,817 & $0,717-0,894$ \\
\hline
\end{tabular}

Tableau 12 : Aires sous la courbe des courbes ROC homolatérales à l'atteinte motrice. 
La comparaison des aires sous la courbe des courbes ROC entre elles montrait que :

- l'aire sous la courbe du SUV putamen postérieur était significativement supérieure à celle du SUV putamen antérieur ( $\mathrm{p}=0,0004)$, à celle du SUV striatum $(\mathrm{p}=0,0002)$ ainsi qu'à celle du SUV noyau caudé $(\mathrm{p}<0,0001)$;

- l'aire sous la courbe du SUV putamen antérieur était significativement supérieure à celle du SUV noyau caudé $(\mathrm{p}<0,0001)$.

- l'aire sous la courbe du SUV striatum était significativement supérieure à celle du SUV noyau caudé ( $\mathrm{p}<0,0001)$.

- l'aire sous la courbe du SUV putamen antérieur n'était pas significativement supérieure à celle du SUV striatum $(\mathrm{p}=0,1111)$

\subsection{Courbes ROC : figures de mérite SOR, COR et POR controlatéraux et homolatéraux à l'atteinte motrice prédominante}

Les courbes ROC ont été établies sur les valeurs des rapports de fixation: SOR, COR, POR antérieur et POR postérieur premièrement sur le côté controlatéral à l'atteinte motrice prédominante et secondairement du côté homolatéral.

\subsubsection{Courbes ROC: SOR, COR, POR du côté controlatéral à l'atteinte motrice} prédominante.

La figure 22 expose les courbes ROC établies sur les valeurs des rapports de fixation : SOR, COR, POR antérieur et POR postérieur du côté homolatéral à l'atteinte motrice prédominante.

Selon l'indice de Youden (tableau 7) nous avons obtenu pour chaque figure de mérite une valeur de sensibilité et de spécificité minimisant le nombre de faux positif et le nombre de faux négatif (selon le point sur la courbe le plus proche de 1). 


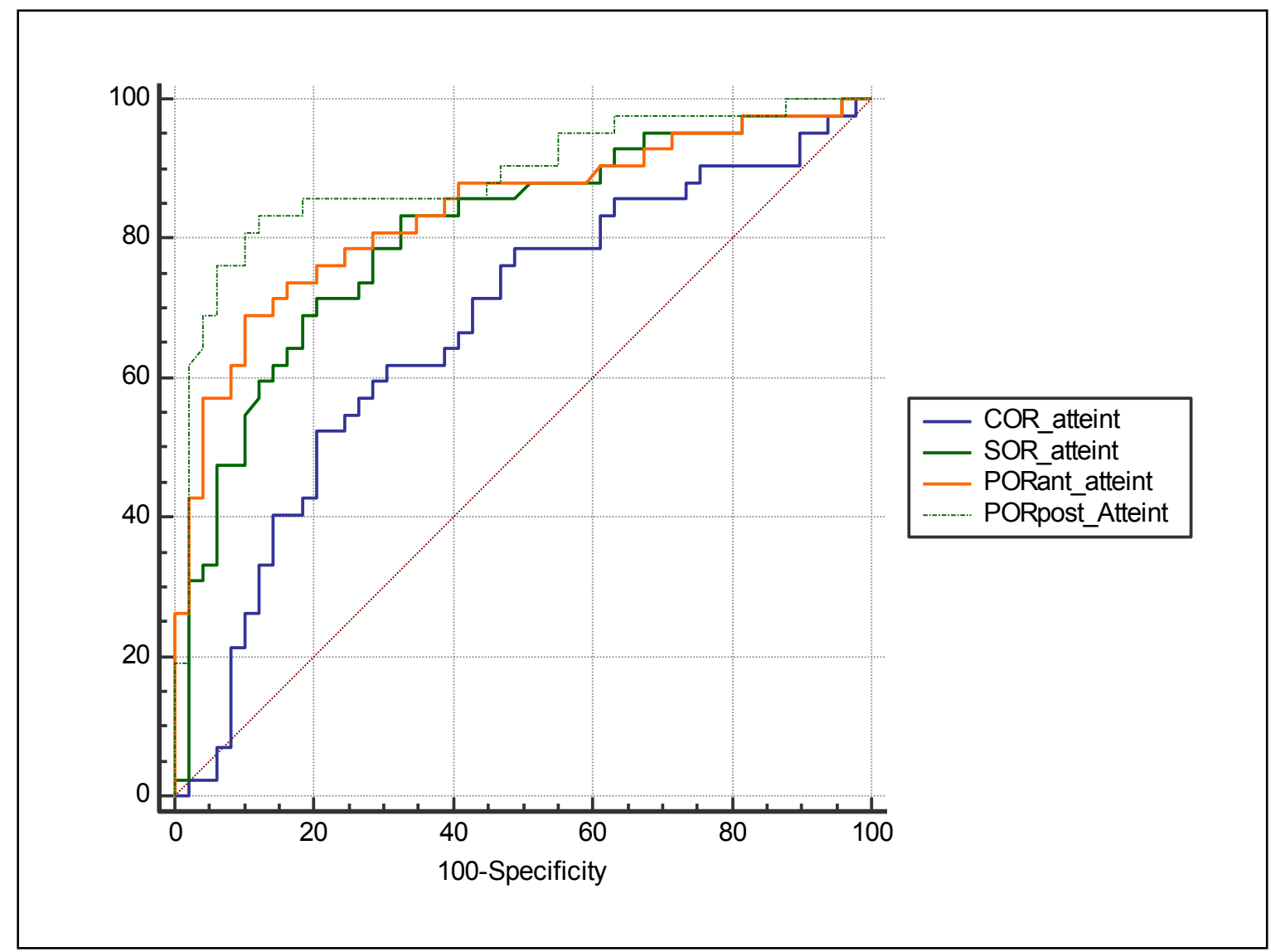

Figure 22 : Courbes ROC: SOR, COR, POR antérieur, POR postérieur sur le côté controlatéral à l'atteinte motrice prédominante.

Le POR postérieur controlatéral à l'atteinte motrice prédominante est la figure de mérite qui permet d'obtenir les meilleures performances diagnostiques. Pour un POR postérieur $\leq 2,44$ la sensibilité est de 83,3\%, et la spécificité de 87, 8\% (index de Youden 0,7109).

Pour le POR antérieur controlatéral à l'atteinte motrice prédominante nous avons obtenu une valeur seuil POR antérieur $\leq 2,51$ qui conduit à une sensibilité de 69,1\% et une spécificité de 89,8\% (index de Youden 0,5884).

Pour le SOR antérieur controlatéral à l'atteinte motrice prédominante nous avons trouvé une valeur seuil de $\mathrm{SOR} \leq 2,73$ qui conduit à une sensibilité de 71,4\% et une spécificité de 79,6\% (index de Youden 0,5102)

Et enfin pour le COR controlatéral à l'atteinte motrice prédominante nous avons obtenu une valeur seuil COR $\leq 2,50$ conduisant à une sensibilité de $52,4 \%$ et une spécificité de 79,60\% (index de Youden 0,3197). Les différents paramètres des 4 courbes sont exposés dans le tableau 13. 


\begin{tabular}{|l|l|l|l|l|l|l|}
\hline $\begin{array}{l}\text { Rapport de } \\
\text { fixation }\end{array}$ & Valeur seuil & Sensibilité & IC 95\% & Spécificité & IC 95\% & $\begin{array}{l}\text { Index de } \\
\text { Youden }\end{array}$ \\
\hline SOR & $\leq 2,73$ & 71,43 & $55,4-84,3$ & 79,59 & $65,7-89,8$ & 0,5102 \\
\hline COR & $\leq 2,50$ & 52,38 & $36,4-68$ & 79,59 & $65,7-89,8$ & 0,3197 \\
\hline $\begin{array}{l}\text { POR } \\
\text { antérieur }\end{array}$ & $\leq 2,51$ & 69,05 & $52,9-82,4$ & 89,80 & $77,8-96,6$ & 0,5884 \\
\hline $\begin{array}{l}\text { POR } \\
\text { postérieur }\end{array}$ & $\leq 2,44$ & 83,33 & $68,6-93,0$ & 87,76 & $75,2-95,4$ & 0,7109 \\
\hline
\end{tabular}

Tableau 13 : Sensibilité et spécificité des différents outils de mesure du côté controlatéral à l'atteinte motrice prédominante

Le tableau 14 expose les aires sous la courbe des 4 courbes ROC précédentes ainsi que leur intervalle de confiance à 95\%.

\begin{tabular}{|l|l|l|}
\hline Variable & AUC & $95 \%$ CI \\
\hline SOR controlatéral & 0,806 & 0,709 to 0,881 \\
\hline COR controlatéral & 0,670 & 0,563 to 0,765 \\
\hline PORant controlatéral & 0,838 & 0,746 to 0,907 \\
\hline PORpost controlatéral & 0,888 & 0,805 to 0,945 \\
\hline
\end{tabular}

Tableau 14 : Aires sous la courbe des courbes ROC controlatérales à l'atteinte motrice.

Les trois courbes POR postérieur, POR antérieur et SOR présentent des aires sous la courbe qui approchent la valeur 1 , respectivement de $0,888,0,838$ et 0,806 . La courbe COR présente une aire sous la courbe plus faible à 0,670.

Lorsque l'on compare les aires sous les courbes entre elles, celle du POR postérieur est significativement supérieure aux 3 autres $(p=0,02, p=0,003$ et $\mathrm{p}<0,0001$ ). Ensuite vient l'aire sous la courbe $\mathrm{du}$ POR antérieur qui est significativement supérieure à la courbe $\operatorname{COR}(\mathrm{p}<0,0001)$ mais qui ne présente pas de différence significative avec celle du SOR $(p=0,07)$. Enfin, l'aire sous la courbe SOR est, elle, significativement supérieure à la courbe COR $(\mathrm{p}<0,0001)$. 


\section{prédominante.}

La figure 23 expose les courbes ROC établies sur les valeurs des rapports de fixation : SOR, COR, POR antérieur et POR postérieur du côté homolatéral à l'atteinte motrice prédominante.

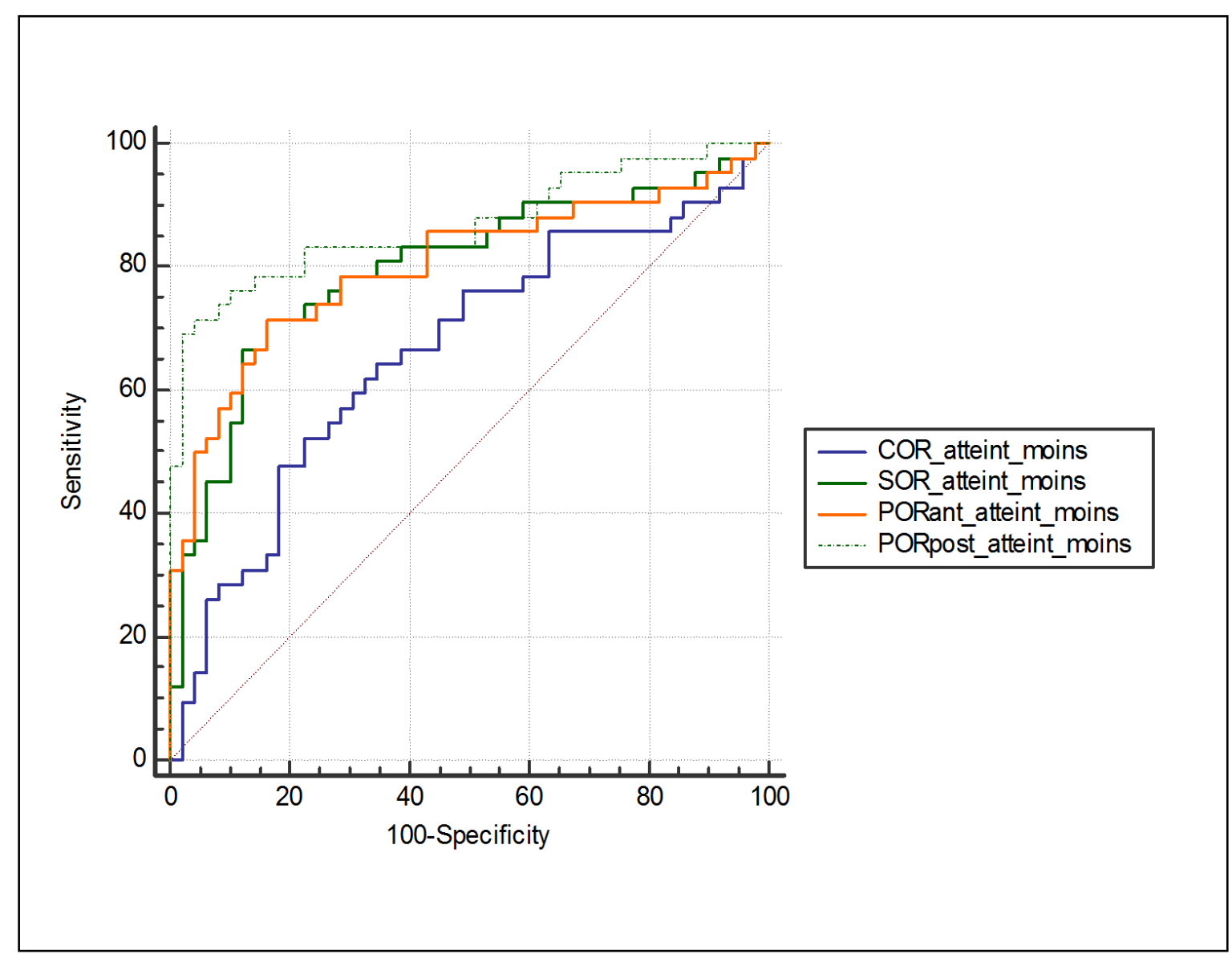

Figure 23 : Courbes ROC : SOR, COR, POR antérieur, POR postérieur sur le côté homolatéral à l'atteinte motrice prédominante.

Le POR postérieur homolatéral à l'atteinte motrice prédominante est la figure de mérite qui obtient également les meilleurs paramètres diagnostiques. Pour un POR postérieur $\leq 2,22$ on obtient une sensibilité de $71,4 \%$ et une spécificité de 95,92\% (index de Youden 0,6735).

Pour le POR antérieur homolatéral à l'atteinte motrice prédominante nous avons obtenu une valeur seuil POR antérieur $\leq 2,68$ qui conduit à une sensibilité de 71,4\% et une spécificité de 83,7\% (index de Youden 0,5510). 
Pour le SOR antérieur homolatéral à l'atteinte motrice prédominante nous avons trouvé une valeur seuil de $\mathrm{SOR} \leq 2,77$ qui conduit à une sensibilité de $71,4 \%$ et une spécificité de 83,7\% (index de Youden 0,5510).

Et enfin pour le COR homolatéral à l'atteinte motrice prédominante nous avons obtenu une valeur seuil COR $\leq 2,55$ conduisant à une sensibilité de $52,4 \%$ et une spécificité de 77,5\% (index de Youden 0,2993).

Le tableau 15 résume l'ensemble des valeurs seuils avec leur sensibilité, leur spécificité avec leur intervalle de confiance respectif et l'indice de Youden.

\begin{tabular}{|l|l|l|l|l|l|l|}
\hline $\begin{array}{l}\text { Rapport de } \\
\text { fixation }\end{array}$ & Valeur seuil & Sensibilité & IC 95\% & Spécificité & IC 95\% & $\begin{array}{l}\text { Index de } \\
\text { Youden }\end{array}$ \\
\hline SOR & $\leq 2,77$ & 71,43 & $55,4-84,3$ & 83,67 & $70,3-92,7$ & 0,5510 \\
\hline COR & $\leq 2,55$ & 52,38 & $36,4-68,0$ & 77,55 & $63,4-88,2$ & 0,2993 \\
\hline $\begin{array}{l}\text { POR } \\
\text { antérieur }\end{array}$ & $\leq 2,68$ & 71,43 & $55,4-84,3$ & 83,67 & $70,3-92,7$ & 0,5510 \\
\hline $\begin{array}{l}\text { POR } \\
\text { postérieur }\end{array}$ & $\leq 2,22$ & 71,43 & $55,4-84,3$ & 95,92 & $86,0-99,5$ & 0,6735 \\
\hline
\end{tabular}

Tableau 15 : Sensibilité et spécificité des différents outils de mesure du côté homolatéral à l'atteinte motrice prédominante

Le tableau 16 expose les aires sous la courbe des 4 courbes ROC précédentes ainsi que leur intervalle de confiance à 95\%.

\begin{tabular}{|l|l|l|}
\hline Variable & AUC & $95 \%$ CI \\
\hline SOR homolatéral & 0,796 & 0,699 to 0,874 \\
\hline COR homolatéral & 0,662 & 0,556 to 0,758 \\
\hline POR antérieur homolatéral & 0,801 & 0,704 to 0,878 \\
\hline POR postérieur homolatéral & 0,867 & 0,780 to 0,929 \\
\hline
\end{tabular}

Tableau 16 : Aires sous la courbe des courbes ROC homolatérales à l'atteinte motrice.

La courbe ROC réalisée pour la figure de mérite POR postérieur obtient la plus grande aire sous la courbe $(0,867$, c'est à dire la plus proche de 1$)$, ensuite vient la courbe du POR antérieur, puis la courbe du SOR et en dernier la courbe du COR 
qui obtient l'aire sous la courbe la plus faible et donc des paramètres diagnostiques inférieurs.

La comparaison des courbes entre-elles montre que l'aire sous la courbe du POR postérieur est significativement supérieure à celle du POR antérieur $(p=0,0022)$ ainsi qu'à celles du SOR $(p=0,003)$ et du COR $(p<0,0001)$. La courbe sur le POR antérieur présente des paramètres significativement supérieurs à ceux de la courbe COR ( $\mathrm{p}<0,0001)$. Par contre il n'y a pas de différence significative entre les deux courbes SOR et POR ant $(\mathrm{p}=0,697)$. Pour finir, la courbe SOR est significativement supérieure à la courbe $\operatorname{COR}(\mathrm{p}<0,0001)$.

Que ce soit du côté homo ou controlatéral à l'atteinte motrice prédominante, la figure de mérite obtenant les meilleures valeurs diagnostiques reste le POR postérieur. On note cependant que du côté controlatéral à l'atteinte motrice prédominante les valeurs diagnostiques sont sensiblement meilleures : sensibilité $83,3 \%$ versus $73,8 \%$ et une aire sous la courbe de 0,888 versus 0,867 .

L'ensemble des outils diagnostiques (analyse visuelle et semi-quantitative) chez les patients normaux et anormaux testés sur les côtés homo et controlatéraux à l'atteinte motrice prédominante sont exposés de façon récapitulative dans le tableau 17. 


\begin{tabular}{|c|c|c|c|c|c|c|c|c|}
\hline & & $\begin{array}{l}\text { Moyen } \\
\text { ne } \\
\text { normal } \\
e\end{array}$ & $\begin{array}{l}\text { Moyen } \\
\text { ne } \\
\text { anorm } \\
\text { ale }\end{array}$ & $p$ & $A U C$ & $\begin{array}{l}\text { Valeur } \\
\text { seuil }\end{array}$ & $\begin{array}{l}\text { Sensibi } \\
\text { lité (\%) }\end{array}$ & $\begin{array}{l}\text { Spécificit } \\
e ́(\%)\end{array}$ \\
\hline $\begin{array}{l}\text { Analyse } \\
\text { visuelle }\end{array}$ & & - & - & - & - & - & 78 & 98 \\
\hline \multirow{4}{*}{$\begin{array}{l}\text { SUV contro } \\
\text { latéraux }\end{array}$} & Striatum & 4,92 & 4,23 & 0,002 & 0,708 & $\leq 4,52$ & 65,79 & 73,33 \\
\hline & $\begin{array}{l}\text { Noyau } \\
\text { caudé }\end{array}$ & 4,36 & 4,14 & 0,265 & 0,598 & $\leq 3,94$ & 47,37 & 75,56 \\
\hline & $\begin{array}{l}\text { Putamen } \\
\text { ant }\end{array}$ & 4,81 & 3,88 & $<0,001$ & 0,742 & $\leq 4,02$ & 63,16 & 77,78 \\
\hline & $\begin{array}{l}\text { Putamen } \\
\text { post }\end{array}$ & 4,64 & 3,33 & $<0,001$ & 0,817 & $\leq 3,37$ & 60,53 & 91,11 \\
\hline \multirow{4}{*}{$\begin{array}{l}\text { SUV homo } \\
\text { latéraux }\end{array}$} & Striatum & 4,89 & 4,31 & 0,007 & 0,698 & $\leq 4,51$ & 68,42 & 73,33 \\
\hline & $\begin{array}{l}\text { Noyau } \\
\text { caudé }\end{array}$ & 4,48 & 4,26 & 0,292 & 0,595 & $\leq 4,25$ & 57,89 & 64,44 \\
\hline & $\begin{array}{l}\text { Putamen } \\
\text { ant }\end{array}$ & 4,83 & 4,11 & 0,001 & 0,715 & $\leq 4,54$ & 71,05 & 68,89 \\
\hline & $\begin{array}{l}\text { Putamen } \\
\text { post }\end{array}$ & 4,64 & 3,46 & $<0,001$ & 0,817 & $\leq 3,7$ & 68,42 & 84,44 \\
\hline \multirow{4}{*}{$\begin{array}{l}\text { Rapports de } \\
\text { fixation } \\
\text { contro } \\
\text { latéraux }\end{array}$} & Striatum & 3,04 & 2,57 & $<0,001$ & 0,806 & $\leq 2,73$ & 71,43 & 79,59 \\
\hline & $\begin{array}{l}\text { Noyau } \\
\text { caudé }\end{array}$ & 2,72 & 2,53 & 0,021 & 0,670 & $\leq 2,50$ & 52,38 & 79,59 \\
\hline & $\begin{array}{l}\text { Putamen } \\
\text { ant }\end{array}$ & 2,98 & 2,37 & $<0,001$ & 0,838 & $\leq 2,51$ & 69,05 & 89,80 \\
\hline & $\begin{array}{l}\text { Putamen } \\
\text { post }\end{array}$ & 2,88 & 2 & $<0,001$ & 0,888 & $\leq 2,44$ & 83,33 & 87,76 \\
\hline \multirow{4}{*}{$\begin{array}{l}\text { Rapports de } \\
\text { fixation } \\
\text { homo } \\
\text { latéraux }\end{array}$} & Striatum & 3,06 & 2,64 & $<0,001$ & 0,796 & $\leq 2,77$ & 71,43 & 83,67 \\
\hline & $\begin{array}{l}\text { Noyau } \\
\text { caudé }\end{array}$ & 2,77 & 2,6 & 0,035 & 0,662 & $\leq 2,55$ & 52,38 & 77,55 \\
\hline & $\begin{array}{l}\text { Putamen } \\
\text { ant }\end{array}$ & 3,01 & 2,51 & $<0,001$ & 0,801 & $\leq 2,68$ & 71,43 & 83,67 \\
\hline & $\begin{array}{l}\text { Putamen } \\
\text { post }\end{array}$ & 2,90 & 2,15 & $<0,001$ & 0,867 & $\leq 2,22$ & 71,43 & 95,92 \\
\hline
\end{tabular}

Tableau 17 : Récapitulatif des outils de mesure diagnostiques 


\section{III/ DISCUSSION}

Le diagnostic de la maladie de Parkinson est, dans la plupart des cas, un diagnostic clinique. Cependant lorsque l'analyse clinique de la symptomatologie reste douteuse, lorsque les symptômes sont peu marqués à un stade précoce ou encore lorsque l'évolution de la pathologie est atypique ou bien lorsque le traitement ne fonctionne pas, les médecins neurologues peuvent être amenés à prescrire un examen complémentaire comme aide au diagnostic.

La médecine nucléaire propose depuis plusieurs dizaines d'années un examen d'imagerie fonctionnelle permettant d'évaluer l'atteinte dégénérative dopaminergique: le 123I-FP-CIT (123I- fluoropropyl-2béta-carbomethoxy3béta(4idodophényl) nortropane) connu sous le nom commercial de DatSCAN® qui a d'ores et déjà démontré son intérêt $(44,45)$. Avec l'arrivée des caméras TEP et des nouveaux radiopharmaceutiques, la scintigraphie cérébrale au ligand DAT a été remplacée dans notre service par la TEP cérébrale à la 18F-FDOPA, offrant une meilleure résolution spatiale d'image et un temps d'acquisition moins long. Ce changement de pratique de routine nous a amenés à nous interroger sur l'avis de nos médecins prescripteurs sur ce nouvel examen et à évaluer dans quelle mesure il les aidait au diagnostic. C'est dans ce but que nous avons construit cette étude clinique prospective monocentrique.

Une des limites de notre étude est de ne pas avoir comparé notre examen à la référence diagnostique incontestable qui est l'examen histopathologique postmortem. Les études comparant le diagnostic clinique au diagnostic autopsique ont montré un taux d'erreur du diagnostic clinique de 20 à $25 \%$ et que le temps nécessaire pour obtenir un diagnostic correct pouvait varier de quelques mois à plusieurs années (46). Nous avons choisi de considérer le diagnostic du neurologue comme référence pour des raisons pratiques évidentes mais aussi pour établir, audelà de la performance diagnostique, l'intérêt concret de l'examen en pratique courante pour le neurologue. Une telle approche s'inscrit dans le cadre d'une 
évaluation des pratiques qui a pour but d'améliorer le service rendu au patient. Nous aurions pu le comparer au DatSCAN® mais cela a été déjà réalisé dans la littérature et plusieurs études ont montré une équivalence entre les deux examens aussi bien pour les stades précoces qu'avancés $(26,27)$. De plus cela aurait été difficile à mettre en œuvre notamment pour les patients qui auraient dû venir deux fois dans le service et subir un examen irradiant supplémentaire.

Afin de retenir le patient comme «sain» ou «pathologique» nous nous sommes basés sur le diagnostic final retenu à l'instant t par le neurologue. Il aurait été intéressant de suivre l'évolution des patients sur 6 mois, 1 an puis 2 ans car le diagnostic peut être amené à changer au cours du suivi. Suivre notre cohorte de patients dans le temps pourra faire l'objet d'une seconde étude prospective dans les mois et années à venir.

L'objet de notre étude a été de sonder chaque médecin neurologue prescrivant l'examen sur une période de 6 mois et ce pour chaque examen prescrit et quelle que soit le type d'activité du médecin. Nous avons eu des médecins exerçant au sein du CHU de Nice, d'autres dans de plus petites structures dites de périphéries, d'autres exerçant une activité mixte ou bien libérale pure. Cela a permis d'évaluer l'intérêt de l'examen quel que soit le type de médecine exercée car on peut supposer que les médecins exerçant au CHU ont accès aux différents examens complémentaires plus facilement, sont moins isolés et peuvent bénéficier plus rapidement de l'expertise d'un confrère en cas de difficulté. Enfin le questionnaire était envoyé quel que soit le résultat de la TEP, qu'elle soit normale ou non.

Lorsqu'un patient présente un syndrome extra-pyramidal, un retard diagnostique ou un mauvais diagnostic peut induire des coûts supplémentaires de santé, un traitement inapproprié et/ou une perte de chances pour le patient. Nous avons montré que selon les neurologues prescripteurs la TEP cérébrale à la $18 \mathrm{~F}$ FDOPA renforce et confirme le diagnostic du médecin prescripteur dans $71 \%$ des cas et élimine des diagnostics différentiels dans 1 cas sur 3. Selon les neurologues l'examen permet aussi de changer le diagnostic dans 1 cas sur 3. Enfin il change la prise en charge du patient de façon probable ou certaine dans $85 \%$ des cas et le 
changement le plus important était l'arrêt ou le début d'un traitement dans 73\% des cas lorsqu'il y avait un changement. En voyant ces chiffres très parlant sur l'intérêt diagnostique de la TEP cérébrale à la 18F-FDOPA, il serait intéressant d'évaluer également son intérêt en termes de santé publique et de réduction des coûts de santé.

Dans 90\% des cas l'examen n'était pas represcrit pour suivre l'évolution, ce qui est en accord avec les recommandations. Cependant nous aurions aimé savoir si dans le cas où l'examen est prescrit une seconde fois pour le même patient, cela correspond plutôt aux cas douteux ou aux examens normaux avec symptômes cliniques équivoques. Cela n'a pas pu ressortir significativement dans notre analyse statistique devant l'échantillon trop faible et il serait intéressant de suivre dans le temps ces patients afin d'évaluer si le diagnostic change au cours du suivi à 1 an, 2 ans voire 5 ans comme évoqué précédemment. L'article de Kuriakosa et al (47) propose effectivement de se servir de la TEP cérébrale à la 18 F-FDOPA pour le monitorage de la progression de la maladie. Cependant se pose la question de l'intérêt de prescrire l'examen à distance lorsque celui-ci est normal en présence de signes cliniques douteux. Dans l'étude de Fahn et al (48), les patients ayant une suspicion clinique de MP avaient un DaTSCAN initial puis après 40 semaines de suivi. Les 19 patients qui n'avaient pas de perte dopaminergique sur l'examen initial avaient également un examen normal à 40 semaines, de plus ils ne répondaient pas au traitement. Toutes les études ayant réalisé un monitorage par TEP à la DOPA montrent que la progression de l'atteinte dopaminergique suit une courbe exponentielle négative, avec une décroissance rapidement évolutive les premières années qui ralentit ensuite avec l'évolution de la maladie et on peut donc penser qu'en présence de symptômes, un examen normal le restera aussi au cours du suivi et que la cause n'est pas une atteinte neuronale dopaminergique.

En ce qui concerne l'impact clinique et diagnostique de la TEP cérébrale à la 18 F-FDOPA nous n'avons trouvé aucune étude similaire à la nôtre dans la littérature pour pouvoir y confronter nos résultats. Cependant il existe plusieurs études sur l'impact clinique et diagnostique du DatSCAN® $(49,50)$. L'étude de Catafau et al (51) a cherché à montrer l'intérêt clinique et diagnostic du DaTSCAN® 
chez 118 patients présentant un syndrome parkinsonien cliniquement incertain. L'examen renforçait la confiance dans le diagnostic, induisant un changement de prise en charge clinique dans $72 \%$ des cas. Il a changé le diagnostic pour 52\% des patients. L'étude la plus récente, datant de mai 2015, de l'équipe de Gayed et al (52) est une étude rétrospective de 57 patients pour lesquels les DatSCAN@ ont été relus par deux observateurs en aveugle afin d'établir l'impact diagnostique de l'examen. Ils ont obtenu une sensibilité de $92 \%$ et une bonne reproductibilité interobservateur. Ils concluent que cet examen aide à confirmer le diagnostic de MP pour les patients cliniquement douteux.

L'interprétation de l'examen par le médecin nucléaire repose pour le moment sur une analyse visuelle. Cette dernière a montré dans notre étude d'excellentes performances diagnostiques (sensibilité 78\% et spécificité 98\%). Son évaluation présente cependant un biais puisque le diagnostic de référence était celui retenu par le neurologue qui le rendait après avoir obtenu les résultats de l'examen. L'analyse visuelle est limitée par le fait qu'il n'existe aucune zone de fixation cérébrale de référence à laquelle on puisse comparer la fixation striatale. En effet ce radiopharmaceutique est spécifique du striatum qui est aussi la zone pathologique à étudier. Ceci nécessite des critères strictes d'interprétation visuelle et d'établir, pour l'analyse semi-quantitative, des seuils de fixation de référence sur une base de sujets normaux/anormaux qui pourra aider à l'interprétation. Dans notre étude nous avons tenté de trouver des seuils de fixation qui pourraient discriminer les patients présentant une dénervation dopaminergique striatale de ceux n'en présentant pas. La caractérisation de ces figures de mérites quantitatives permettrait de pallier les limites de la méthode visuelle qui présente comme inconvénient un manque de reproductibilité inter et intra-opérateur. Nous avons relu un échantillon de trente examens tirés au sort de façon aléatoire pour évaluer notre reproductibilité interopérateur sur l'analyse quantitative. Nous avons obtenu une excellente reproductibilité inter-opérateur et celle-ci pourrait être encore accrue à l'aide de logiciels semi-automatisés comme il en existe actuellement pour interpréter le DatSCAN@ (53) . Il serait très intéressant de tester ce type de logiciel en l'adaptant à la TEP cérébrale à la 18 F-FDOPA. Nous prenons pour exemple le logiciel DaTsoft3D* qui fait une reconstruction itérative OSEMR des images, utilise une échelle de 
couleur quantitative basée sur l'activité cérébrale non spécifique et réalise un recalage sur un modèle 3D du striatum. Il permet une correction de l'effet de volume partiel adapté à la résolution spatiale de l'image ainsi qu'une quantification du potentiel de liaison. L'intérêt de ce type de logiciel est qu'il permet une comparaison de l'examen à une base de données de patients sains comme nous l'utilisons déjà en routine dans notre service pour la TEP cérébrale au 18F-FDG qui a récemment remplacé la scintigraphie cérébrale de perfusion. En utilisant notre cohorte de patients il serait intéressant d'essayer d'adapter ce type de logiciel à la TEP cérébrale à la 18F-FDOPA et cela fera l'objet d'une étude subsidiaire.

Chang et al(16) ont cependant comparé la méthode manuelle à la méthode automatisée et n'ont pas trouvé de différence significative entre les deux méthodes de mesure. En effet les valeurs des rapports de fixation obtenus par la méthode d'analyse automatique étaient hautement corrélées à celles obtenues par la méthode manuelle. La méthode automatisée présenterait comme avantage selon eux un gain de temps et donc de coût.

Afin d'améliorer les performances de l'analyse visuelle bien que déjà satisfaisantes, l'enjeu est donc de définir des outils de quantification simples et reproductibles permettant de discriminer les patients malades dès les stades précoces de la maladie. C'est ce que nous avons fait dans notre étude grâce à notre cohorte de patient composée pour moitié du sujet sain et pour moitié de sujets atteints. Nous avons établi à l'aide des courbes ROC des seuils de rapports de fixation en dessous desquels le patient est à considérer comme atteint. La figure de mérite la plus discriminante était le POR postérieur (putamen postérieur occipital ratio) du côté controlatéral à l'atteinte motrice prédominante qui avait une aire sous la courbe de 0,888 . Pour un seuil de rapport de fixation fixé à 2,44 , nous obtenons une sensibilité de 83,4\% et une spécificité de 87,8\%. Ensuite venait le POR antérieur avec un seuil de 2,5 (sensibilité 69\% et spécificité 89,8\%) puis le SOR avec un seuil de 2,73 (sensibilité 71,4\% et spécificité 89,8\%). Le rapport de fixation réalisé sur le COR avait les moins bons paramètres diagnostiques. Nos résultats correspondent à ceux retrouvés dans la littérature. Il est intéressant de remarquer que la spécificité $(87,8 \%)$ de la figure de mérite la plus performante : POR postérieur controlatéral 
reste cependant inférieure à celle de l'analyse visuelle (98\%), et la sensibilité est à peine supérieure $(83,3 \%$ versus $78 \%)$.

L'étude de Oehme et al (54), bien que réalisée avec une quantification dynamique, trouve également les meilleurs paramètres diagnostiques pour distinguer les patients sains des patients MP au niveau du POR controlatéral à l'atteinte motrice prédominante. Par contre ils ne distinguent pas dans cette étude le putamen antérieur du putamen postérieur de même que Pavese et al (23) qui trouvent des résultats similaires sur le putamen en suivant des patients MP pendant 3 ans. Dans notre étude nous avons distingué le putamen postérieur du putamen antérieur comme suggéré par Jokinen et al (14). En effet le putamen postérieur se révèle être la zone de mesure la plus discriminante de même que dans l'étude de Brück et al (21). Il permet de distinguer les patients atteints des patients sains de façon la plus précoce au niveau controlatéral à l'atteinte motrice prédominante. Nurmi et al (20) ont également observé une diminution plus importante de la fixation dans le putamen postérieur plutôt que dans le putamen antérieur et le noyau caudé. Au niveau physiopathologique on peut expliquer cela par le fait que le putamen postérieur reçoit des projections de la partie ventro-latérale de la substance noire qui est la région qui subit l'atteinte dégénérative la plus importante dans les MP $(55,56)$.

Dans l'étude la plus récente de Eshuis et al (27) qui compare directement la TEP cérébrale à la 18 F-DOPA et le DatSCAN®, il a été défini des valeurs de rapport de fixation moyennes chez les sujets contrôles $(n=10)$ qui sont très proches de nos valeurs obtenues sur nos patients «normaux» $(n=45)$ : SOR striatum 2,69 dans leur étude versus 3,04 pour la nôtre, COR 2,69 pour leur étude versus 2,74 dans notre étude et POR 2,70 versus 2,88 pour le POR post et 2,99 pour le POR ant dans notre étude.

Jokinen et al (14) ont également trouvé la plus grande aire sous la courbe pour l'outil de mesure POR postérieur controlatéral à l'atteinte motrice prédominante. Elle était sensiblement supérieure à la nôtre : 0,994 contre 0,888 dans notre étude.

Enfin l'étude des rapports de fixation spécifique sur non spécifique (occipitale), se révèle être une méthode plus simple et plus reproductible (37) que la 
méthode de calcul de constante Ki qui s'obtient par une acquisition dynamique fastidieuse et difficilement réalisable en routine. Cependant cette dernière méthode est plus sensible aux variations interindividuelles et aux changements au cours de l'évolution (57).

Dans notre groupe de patients dit «normaux » basé sur le diagnostic final retenu par le neurologue nous avions des patients présentant un tremblement essentiel ou iatrogène. Nous avons pu les regrouper ainsi car les données de la littérature et notamment l'étude de Cummings et al (46) sont convergentes à ce sujet: il n'y a pas de différence significative entre les sujets témoins et les sujets présentant un tremblement essentiel. Cela pouvait être attendu puisque au niveau physiopathologique il n'y a pas d'atteinte des neurones dopaminergiques dans le tremblement essentiel ces patients peuvent donc être considérés comme des sujets témoins.

Alors que pour le DatSCAN® les données de la littérature objectivent une diminution de la fixation striatale spécifique de 4\% tous les 10 ans chez les sujets normaux, cet effet lié à l'âge n'est pas retrouvé en TEP à la 18 F-FDOPA (27). Pour ce dernier examen on ne retrouve pas de changement significatif (moins de 1\%) dans les études de Morrish et al (28) et Nurmi et al (20) qui ont évalué la progression de l'atteinte au cours du temps. De même dans notre étude nous avons cherché à évaluer s'il existait une différence du niveau de fixation dans notre cohorte de patients «normaux» selon l'âge. L'âge médian était de 73,7 ans et nous n'avons trouvé aucune différence significative des SUV ou bien des rapports de fixation entre les sujets de moins de 73,7 ans et ceux de plus de 73,7 ans. Ces résultats sont concordants avec d'autres résultats de la littérature (58-60).

Une autre limite de notre étude est qu'elle ne présentait pas de critères d'inclusion ou d'exclusion prédéfinis et que tous les patients bénéficiant d'une TEP cérébrale à la 18F-FDOPA ont été inclus. Il serait intéressant de réaliser une étude avec critères d'inclusion stricts se basant sur des échelles de score clinique telles que l'UPDRS (Unified Parkinson's Disease Rating Scale) recommandée par la HAS et en corrélant le degré de l'atteinte neuronale dopaminergique à la clinique comme 
ont réussi à le réaliser l'équipe de Picco et al (61) dans leur inclusion de patients. En effet dans leur étude cela avait un intérêt direct puisqu'ils ont étudié et cherché à corréler la fixation extra-striatale de la 18F-FDOPA à celle de l'atteinte cognitive des patients MP. Selon eux, le lobe frontal médial, le gyrus cingulaire antérieur et le gyrus frontal médian seraient impliqués dans l'atteinte des fonctions exécutives et du langage chez les patients MP précoces. Il semble donc que l'atteinte des neurones dopaminergiques ne se limite pas au striatum et les champs d'étude de cette maladie neuro-dégénérative par la TEP cérébrale à la 18 F-DOPA sont encore multiples. 


\section{CONCLUSION}

Notre revue de la littérature montre que dans un contexte de recherche la TEP cérébrale à la 18 F-FDOPA est un examen sensible et fiable dans le diagnostic des syndromes parkinsoniens. Malgré les controverses liées aux mécanismes compensateurs qui diminueraient la sensibilité de la TEP cérébrale à la 18 F-FDOPA dans le diagnostic des MP précoces, les données de la littérature montrent que ses performances diagnostiques sont équivalentes (voire supérieure) à celle de la TEMP FP-CIT y compris pour les stades précoces de la maladie (27). En effet, la TEP cérébrale à la 18F-FDOPA est un examen ayant une sensibilité au moins équivalente à celle de la TEMP FP-CIT, elle offre un protocole d'acquisition plus rapide et bénéficie d'une modalité d'imagerie TEP ce qui augmente la sensibilité et la résolution spatiale des images et nous permet de proposer à nos correspondants des images de qualité supérieure. De plus, la 18 F-FDOPA est un radiopharmaceutique dont le rôle dans l'imagerie oncologique (tumeurs neuroendocrines, tumeurs cérébrales, phéochromocytome et paragangliome) est devenu indéniable et a toute sa place dans un service de médecine nucléaire en 2015.

Notre étude, en lien avec l'expérience de notre service de médecine nucléaire, a permis d'apporter la preuve que la TEP cérébrale à la 18 F-FDOPA est un examen réalisable en routine et malgré les difficultés d'approvisionnement du radiopharmaceutique, nous réussissons à réaliser cet examen sur des vacations dédiées une à deux fois par semaine. Depuis l'installation de notre caméra TEP en juillet 2012 nous avons réalisé 1004 TEP cérébrales à la 18F-FDOPA (à la date du 21/08/2015) dans le diagnostic des syndromes parkinsoniens.

L'utilité clinique de la TEP cérébrale à la 18F-FDOPA dans le diagnostic des syndromes parkinsoniens n'avait jamais été évaluée auparavant et notre étude montre que cet examen renforce la confiance dans le diagnostic et permet de confirmer le diagnostic dans $71,4 \%$ des cas. Il change la prise en charge du patient dans $57 \%$ des cas avec un changement de thérapeutique dans $73 \%$ des cas. Cet examen permet aussi de modifier le diagnostic pour plus de $30 \%$ des patients. La 
TEP cérébrale à la 18F-FDOPA apparait être un élément majeur dans la démarche diagnostique des syndromes parkinsoniens dès les stades précoces de la maladie.

Cet examen est utilisé depuis plus de vingt ans pour évaluer les syndromes parkinsoniens en recherche clinique et notre étude montre la faisabilité d'une analyse semi-quantitative en routine clinique. En effet, l'analyse visuelle bien que présentant d'excellentes performances diagnostiques avec une spécificité supérieure à celle de l'analyse semi-quantitative et une sensibilité quasiment équivalente, présente quelques limites avec notamment un manque de reproductibilité intra et inter-opérateur. Développer une analyse semi-quantitative de routine apportera plus de robustesse à cet examen en limitant les biais d'interprétation.

Dans notre étude nous avons pu établir des seuils de rapports de fixation permettant de distinguer les patients atteints des patients sains, le POR postérieur (rapport de fixation spécifique du putamen postérieur sur non spécifique au niveau de l'occiput) du côté controlatéral à l'atteinte motrice prédominante à un seuil de 2,44 obtient les meilleurs paramètres diagnostiques avec une sensibilité de $83 \%$ et une spécificité de 88\%. L'intérêt de cette analyse semi-quantitative est donc de renforcer la reproductibilité de l'examen en fixant des seuils diagnostiques robustes et l'utilisation de logiciels d'analyse automatique offre une perspective intéressante qui fera l'objet d'une prochaine étude sur une plus grande cohorte de patients. 


\section{BIBLIOGRAPHIE}

1. Darcourt J, Booij J, Tatsch K, Varrone A, Vander Borght T, Kapucu OL, et al. EANM procedure guidelines for brain neurotransmission SPECT using (123)Ilabelled dopamine transporter ligands, version 2. Eur J Nucl Med Mol Imaging. févr 2010;37(2):443-50.

2. Van Laere K, Varrone A, Booij J, Vander Borght T, Nobili F, Kapucu OL, et al. EANM procedure guidelines for brain neurotransmission SPECT/PET using dopamine D2 receptor ligands, version 2. Eur J Nucl Med Mol Imaging. févr 2010;37(2):434-42.

3. Gupta D, Kuruvilla A. Vascular parkinsonism: what makes it different? Postgrad Med J. déc 2011;87(1034):829-36.

4. Corvol J-C, Hunot S. Maladie de Parkinson, thématiques, neurosciencessciences-cognitives-neurologie-psychiatrie. Inserm.fr. févr 2015;

5. Fahn S. Parkinson's disease: 10 years of progress, 1997-2007. Mov Disord Off J Mov Disord Soc. 2010;25 Suppl 1:S2-14.

6. Elbaz A, Clavel J, Rathouz PJ, Moisan F, Galanaud J-P, Delemotte B, et al. Professional exposure to pesticides and Parkinson disease. Ann Neurol. oct 2009;66(4):494-504.

7. Darcourt J, Schiazza A, Sapin N, Dufour M, Ouvrier MJ, Benisvy D, et al. 18FFDOPA PET for the diagnosis of parkinsonian syndromes. Q J Nucl Med Mol Imaging Off Publ Ital Assoc Nucl Med AIMN Int Assoc Radiopharmacol IAR Sect Soc Radiopharm Chem Biol. déc 2014;58(4):355-65.

8. Leenders KL, Palmer AJ, Quinn N, Clark JC, Firnau G, Garnett ES, et al. Brain dopamine metabolism in patients with Parkinson's disease measured with positron emission tomography. J Neurol Neurosurg Psychiatry. août 1986;49(8):853- 60. 9. Firnau G, Garnett ES, Chirakal R, Sood S, Nahmias C, Schrobilgen G. [18F]fluoro-L-dopa for the in vivo study of intracerebral dopamine. Int J Rad Appl Instrum [A]. 1986;37(8):669-75.

10. Hoffman JM, Melega WP, Hawk TC, Grafton SC, Luxen A, Mahoney DK, et al. The Effects of Carbidopa Administration on 6-[18F]Fluoro-L-DOPA Kinetics in Positron Emission Tomography. J Nucl Med. 8 janv 1992;33(8):1472-7.

11. Brown WD, Oakes TR, DeJesus OT, Taylor MD, Roberts AD, Nickles RJ, et al. Fluorine-18-fluoro-L-DOPA dosimetry with carbidopa pretreatment. J Nucl Med Off Publ Soc Nucl Med. nov 1998;39(11):1884-91.

12. Guignard R, Schiazza A, Ochin A, Darcourt J. Quantification de l'activité dopaminergique striatale en TEP à la 18F-DOPA : quels paramètres choisir en routine ? Médecine Nucl. avr 2014;38(2):91-9.

13. Scherman D, Desnos C, Darchen F, Pollak P, Javoy-Agid F, Agid Y. Striatal dopamine deficiency in Parkinson's disease: role of aging. Ann Neurol. oct 1989;26(4):551-7.

14. Jokinen P, Helenius H, Rauhala E, Brück A, Eskola O, Rinne JO. Simple ratio analysis of 18F-fluorodopa uptake in striatal subregions separates patients with early Parkinson disease from healthy controls. J Nucl Med Off Publ Soc Nucl Med. juin 2009;50(6):893-9.

15. Dhawan V, Ma Y, Pillai V, Spetsieris P, Chaly T, Belakhlef A, et al. Comparative analysis of striatal FDOPA uptake in Parkinson's disease: ratio method versus graphical approach. J Nucl Med Off Publ Soc Nucl Med. oct 2002;43(10):1324-30. 16. Chang I-C, Lue K-H, Hsieh H-J, Liu S-H, Kao C-HK. Automated striatal uptake 
analysis of 18F-FDOPA PET images applied to Parkinson's disease patients. Ann Nucl Med. 1 déc 2011;25(10):796-803.

17. Brooks DJ. The early diagnosis of parkinson's disease. Ann Neurol. 1 sept 1998;44(S1):S10-8.

18. Brooks DJ, Ibanez V, Sawle GV, Quinn N, Lees AJ, Mathias CJ, et al. Differing patterns of striatal 18F-dopa uptake in Parkinson's disease, multiple system atrophy, and progressive supranuclear palsy. Ann Neurol. 1 oct 1990;28(4):547-55.

19. O. Rinne J, Nurmi E, Ruottinen HM, Bergman J, Eskola 0, Solin 0. [18F]FDOPA and $[18 \mathrm{~F}] \mathrm{CFT}$ are both sensitive PET markers to detect presynaptic dopaminergic hypofunction in early Parkinson's disease. Synapse. 1 juin 2001;40(3):193-200.

20. Nurmi E, Ruottinen HM, Bergman J, Haaparanta M, Solin O, Sonninen P, et al. Rate of progression in Parkinson's disease: a 6-[18F]fluoro-L-dopa PET study. Mov Disord Off J Mov Disord Soc. juill 2001;16(4):608-15.

21. Brück A, Aalto S, Rauhala E, Bergman J, Marttila R, Rinne JO. A follow-up study on 6-[18F]fluoro-L-dopa uptake in early Parkinson's disease shows nonlinear progression in the putamen. Mov Disord Off J Mov Disord Soc. 15 mai 2009;24(7):1009-15.

22. Hilker R, Schweitzer K, Coburger S, et al. NOnlinear progression of parkinson disease as determined by serial positron emission tomographic imaging of striatal fluorodopa f 18 activity. Arch Neurol. 1 mars 2005;62(3):378-82.

23. Pavese N, Rivero-Bosch M, Lewis SJ, Whone AL, Brooks DJ. Progression of monoaminergic dysfunction in Parkinson's disease: a longitudinal 18F-dopa PET study. NeuroImage. 1 juin 2011;56(3):1463-8.

24. Morrish PK, Sawle GV, Brooks DJ. Clinical and [18F] dopa PET findings in early Parkinson's disease. J Neurol Neurosurg Psychiatry. 12 janv 1995;59(6):597600 .

25. Nagasawa H, Saito H, Kogure K, Hatazawa J, Itoh M, Fujiwara T, et al. 6[18F]fluorodopa metabolism in patients with hemiparkinsonism studied by positron emission tomography. J Neurol Sci. avr 1993;115(2):136-43.

26. Eshuis SA, Maguire RP, Leenders KL, Jonkman S, Jager PL. Comparison of FPCIT SPECT with F-DOPA PET in patients with de novo and advanced Parkinson's disease. Eur J Nucl Med Mol Imaging. févr 2006;33(2):200-9.

27. Eshuis SA, Jager PL, Maguire RP, Jonkman S, Dierckx RA, Leenders KL. Direct comparison of FP-CIT SPECT and F-DOPA PET in patients with Parkinson's disease and healthy controls. Eur J Nucl Med Mol Imaging. mars 2009;36(3):454-62.

28. Morrish PK, Sawle GV, Brooks DJ. An [18F]dopa-PET and clinical study of the rate of progression in Parkinson's disease. Brain J Neurol. avr 1996;119 ( Pt 2):58591.

29. Vingerhoets FJ, Snow BJ, Lee CS, Schulzer M, Mak E, Calne DB. Longitudinal fluorodopa positron emission tomographic studies of the evolution of idiopathic parkinsonism. Ann Neurol. nov 1994;36(5):759-64.

30. Burn DJ, Sawle GV, Brooks DJ. Differential diagnosis of Parkinson's disease, multiple system atrophy, and Steele-Richardson-Olszewski syndrome: discriminant analysis of striatal 18F-dopa PET data. J Neurol Neurosurg Psychiatry. mars 1994;57(3):278-84.

31. Sawle GV, Brooks DJ, Marsden CD, Frackowiak RS. Corticobasal degeneration. A unique pattern of regional cortical oxygen hypometabolism and striatal fluorodopa uptake demonstrated by positron emission tomography. Brain J Neurol. févr 1991;114 ( Pt 1B):541-56. 
32. Ghaemi M, Hilker R, Rudolf J, Sobesky J, Heiss W. Differentiating multiple system atrophy from Parkinson's disease: contribution of striatal and midbrain MRI volumetry and multi-tracer PET imaging. J Neurol Neurosurg Psychiatry. nov 2002;73(5):517-23.

33. Laureys S, Salmon E, Garraux G, Peigneux P, Lemaire C, Degueldre C, et al. Fluorodopa uptake and glucose metabolism in early stages of corticobasal degeneration. J Neurol. déc 1999;246(12):1151-8.

34. Turjanski N, Lees AJ, Brooks DJ. Dopaminergic function in patients with posttraumatic parkinsonism: an 18F-dopa PET study. Neurology. juill 1997;49(1):183-9.

35. Hu XS, Okamura N, Arai H, Higuchi M, Matsui T, Tashiro M, et al. 18Ffluorodopa PET study of striatal dopamine uptake in the diagnosis of dementia with Lewy bodies. Neurology. 28 nov 2000;55(10):1575-7.

36. Klein JC, Eggers C, Kalbe E, Weisenbach S, Hohmann C, Vollmar S, et al. Neurotransmitter changes in dementia with Lewy bodies and Parkinson disease dementia in vivo. Neurology. 16 mars 2010;74(11):885-92.

37. Puñal-Riobóo J, Serena-Puig A, Varela-Lema L, Alvarez-Páez AM, RuanoRavina A. [Clinical utility of (18)F-DOPA-PET in movement disorders. A systematic review]. Rev Esp Med Nucl. juin 2009;28(3):106-13.

38. Langston JW, Langston EB, Irwin I. MPTP-induced parkinsonism in human and non-human primates--clinical and experimental aspects. Acta Neurol Scand Suppl. 1984;100:49-54.

39. Ribeiro M-J, Vidailhet M, Loc'h C, Dupel C, Nguyen JP, Ponchant M, et al. Dopaminergic function and dopamine transporter binding assessed with positron emission tomography in Parkinson disease. Arch Neurol. avr 2002;59(4):580-6. 40. Nandhagopal R, Kuramoto L, Schulzer M, Mak E, Cragg J, McKenzie J, et al. Longitudinal evolution of compensatory changes in striatal dopamine processing in Parkinson's disease. Brain J Neurol. nov 2011;134(Pt 11):3290-8.

41. Lee CS, Samii A, Sossi V, Ruth TJ, Schulzer M, Holden JE, et al. In vivo positron emission tomographic evidence for compensatory changes in presynaptic dopaminergic nerve terminals in Parkinson's disease. Ann Neurol. avr 2000;47(4):493-503.

42. Aupee O, Bonardel G, Gontier O, Montravers F. Guide pour la rédaction de protocoles pour la tomographie par émission de positons (TEP) à la 6-fluoro-[18F]L-dihydroxyphénylalanine ou 6-f luoro-[18F]-L-DOPA ([18F]-FDOPA). Soc Fr Med Nucl 2011.

43. DeLong ER, DeLong DM, Clarke-Pearson DL. Comparing the Areas under Two or More Correlated Receiver Operating Characteristic Curves: A Nonparametric Approach. Biometrics. 1 sept 1988;44(3):837-45.

44. Booij J, Tijssen Marina A. J., Berendse Henk W. Clinical Applications of [123I]FP-CIT SPECT Imaging. In: PET and SPECT in Neurology. Dierckx R, Otte A, de Vries E, van Waarde A, Leenders K.; 2014. p. pp 719-36.

45. Vlaar AM, van Kroonenburgh MJ, Kessels AG, Weber WE. Meta-analysis of the literature on diagnostic accuracy of SPECT in parkinsonian syndromes. BMC Neurol. 1 sept 2007;7(1):27.

46. Cummings JL, Henchcliffe C, Schaier S, Simuni T, Waxman A, Kemp P. The role of dopaminergic imaging in patients with symptoms of dopaminergic system neurodegeneration. Brain. 11 janv 2011;134(11):3146-66.

47. Kuriakose R, Stoessl AJ. Imaging the nigrostriatal system to monitor disease 
progression and treatment-induced complications. Prog Brain Res. 2010;184:17792.

48. Fahn S, Oakes D, Shoulson I, Kieburtz K, Rudolph A, Lang A, et al. Levodopa and the progression of Parkinson's disease. N Engl J Med. 9 déc 2004;351(24):2498508.

49. Kupsch AR, Bajaj N, Weiland F, Tartaglione A, Klutmann S, Buitendyk M, et al. Impact of DaTscan SPECT imaging on clinical management, diagnosis, confidence of diagnosis, quality of life, health resource use and safety in patients with clinically uncertain parkinsonian syndromes: a prospective 1-year follow-up of an open-label controlled study. J Neurol Neurosurg Psychiatry. juin 2012;83(6):620-8.

50. Seifert KD, Wiener JI. The impact of DaTscan on the diagnosis and management of movement disorders: A retrospective study. Am J Neurodegener Dis. 2013;2(1):29-34.

51. Catafau AM, Tolosa E, DaTSCAN Clinically Uncertain Parkinsonian Syndromes Study Group. Impact of dopamine transporter SPECT using 123I-Ioflupane on diagnosis and management of patients with clinically uncertain Parkinsonian syndromes. Mov Disord Off J Mov Disord Soc. oct 2004;19(10):1175-82.

52. Gayed I, Joseph U, Fanous M, Wan D, Schiess M, Ondo W, et al. The impact of DaTscan in the diagnosis of Parkinson disease. Clin Nucl Med. mai 2015;40(5):390-3. 53. Gantet P GE. Quantification auto-calibrée de la neurotransmission dopaminergique en TEMP : évaluation de DaTsoft3D. Médecine Nucl.

2014;38(3):133.

54. Oehme L, Perick M, Beuthien-Baumann B, Wolz M, Storch A, Löhle M, et al. Comparison of dopamine turnover, dopamine influx constant and activity ratio of striatum and occipital brain with ${ }^{18} \mathrm{~F}$-dopa brain PET in normal controls and patients with Parkinson's disease. Eur J Nucl Med Mol Imaging. août 2011;38(8):1550-9.

55. Damier P, Hirsch EC, Agid Y, Graybiel AM. The substantia nigra of the human brain. II. Patterns of loss of dopamine-containing neurons in Parkinson's disease. Brain J Neurol. août 1999;122 ( Pt 8):1437-48.

56. Kish SJ, Shannak K, Hornykiewicz O. Uneven pattern of dopamine loss in the striatum of patients with idiopathic Parkinson's disease. Pathophysiologic and clinical implications. N Engl J Med. 7 avr 1988;318(14):876-80.

57. Sawle GV, Playford ED, Burn DJ, Cunningham VJ, Brooks DJ. Separating Parkinson's disease from normality. Discriminant function analysis of fluorodopa F 18 positron emission tomography data. Arch Neurol. mars 1994;51(3):237-43.

58. Ishikawa T, Dhawan V, Kazumata K, Chaly T, Mandel F, Neumeyer J, et al. Comparative nigrostriatal dopaminergic imaging with iodine-123-beta CITFP/SPECT and fluorine-18-FDOPA/PET. J Nucl Med Off Publ Soc Nucl Med. nov 1996;37(11):1760-5.

59. Sawle GV, Colebatch JG, Shah A, Brooks DJ, Marsden CD, Frackowiak RS. Striatal function in normal aging: implications for Parkinson's disease. Ann Neurol. déc 1990;28(6):799-804.

60. Eidelberg D, Takikawa S, Dhawan V, Chaly T, Robeson W, Dahl R, et al. Striatal 18F-dopa uptake: absence of an aging effect. J Cereb Blood Flow Metab Off J Int Soc Cereb Blood Flow Metab. sept 1993;13(5):881-8.

61. Picco A, Morbelli S, Piccardo A, Arnaldi D, Girtler N, Brugnolo A, et al. Brain (18)F-DOPA PET and cognition in de novo Parkinson's disease. Eur J Nucl Med Mol Imaging. juin 2015;42(7):1062-70. 


\title{
ANNEXES
}

\section{A. Annexe 1 : Questionnaire envoyé aux médecins neurologues prescripteurs}

\author{
EXEMPLAIRE A ENVOYER AU MEDECIN PRESCRIPTEUR : Dr
}

QUESTIONNAIRE D’EVALUATION DE L'APPORT CLINIQUE DE LA TEP CEREBRALE A LA 18 F-DOPA DANS LES SYNDROMES PARKINSONIENS AUPRES DES MEDECINS NEUROLOGUES PRESCRIPTEURS

Merci de trouver ci-dessous, un questionnaire qui me permettra grâce à votre contribution d'effectuer le recueil de données pour mon travail de thèse. Celui-ci porte sur l'intérêt diagnostique et clinique de la TEP cérébrale à la 18 F-DOPA dans la maladie de Parkinson auprès des médecins neurologues. Sur une durée de 6 mois, chaque résultat d'examen vous sera communiqué avec ce questionnaire que vous pourrez nous retourner dans le service de Médecine Nucléaire du Centre Antoine Lacassagne de Nice par courrier (enveloppe affranchie jointe), par fax (04.92.03.11.48) ou par mail (aurelie.schiazza@gmail.com). Les données seront traitées statistiquement de façon anonyme et les résultats vous seront communiqués prioritairement. En vous remerciant par avance pour le temps que vous voudrez bien accorder à ce questionnaire. Je suis à votre disposition pour toute information complémentaire. Aurélie Schiazza, Interne de Médecine Nucléaire

Nom et Prénom du patient :

Examen réalisé dans le service de Médecine Nucléaire du CAL de Nice, en date du : ...................

\section{A/INDICATION}

1) Latéralisation de la symptomatologie motrice : $\square$ Droite unilatérale $\square$ Gauche unilatérale $\square$ Bilatérale $G>D \quad \square$ Bilatérale $D>G \quad \square$ Bilatérale symétrique

2) Présence de symptômes non-moteurs: $\square$ Oui $\square$ Non $\square$ Non évalué Si oui (plusieurs réponses possibles) : $\square$ troubles psy $\square$ douleurs $\square$ troubles cognitifs $\square$ dépression $\square$ troubles de l'équilibre $\square$ dysautonomie $\quad \square$ autres :...

3) Début de la symptomatologie: $\square<6$ mois $\square 6$ mois -2 ans $\square 2$ ans -5 ans $\square>5$ ans

4) L'atteinte de la voie dopaminergique striatale AVANT la TEP était:
$\square$ improbable
$\square$ peu probable
$\checkmark$ probable
$\square$ très probable

B/ DIAGNOSTIC

5) Hypothèse diagnostique initiale pré-TEP (plusieurs réponses possibles): $\square$ Maladie de Parkinson précoce $\square$ Maladie de Parkinson avancée $\square$ AMS $\square$ DCB $\square$ Parkinson vasculaire $\square$ Démence à corps de Lewy $\square$ PSP $\square$ Syndrome extra-pyramidal atypique $\quad \square$ latrogénie $\quad$ Tremblement essentiel $\square$ origine psychogène $\quad \square$ Autre

6) Y-a-t-il une corrélation entre l'aspect clinique et le résultat de la TEP: $\quad \square$ oui $\quad \square$ non

7) Diagnostic final que vous retenez: $\square$ Maladie de Parkinson précoce $\square$ Maladie de Parkinson avancée $\square$ AMS $\square$ DCB $\square$ Parkinson vasculaire $\square$ Démence à corps de Lewy $\square$ PSP $\square$ Syndrome extra-pyramidal atypique $\square$ latrogénie $\square$ Tremblement essentiel $\square$ origine psychogène $\square$ Autre :

C/ EVALUATION DE L'INTERET DE LA TEP PAR LE NEUROLOGUE, chez ce patient précisément :

8) Dans quelle mesure le résultat vous aide-t-il pour le diagnostic de votre patient (plusieurs réponses possibles):
$\square$ il est source de confusion
$\square$ il n'apporte rien
$\square$ il renforce ma confiance dans mon diagnostic
$\square$ il confirme mon diagnostic
$\square$ il élimine des diagnostics différentiels mais ne me permet pas de faire le
diagnostic positif

9) Le résultat de la TEP DOPA induira-t-il un changement de prise en charge chez ce patient :

$\square$ non $\square c^{\prime}$ est peu probable $\square c^{\prime}$ est probable $\square c^{\prime}$ est certain

et dans quelle(s) mesure(s) (plusieurs réponses possibles): $\square$ arrêt/début d'un traitement $\square$ demande d'autres examens complémentaires $\square$ modification du suivi $\square$ demande d'ALD, APA, tutelle $\square$ autres mesures :......

10) Prescrirez-vous de nouveau cet examen chez ce patient, pour suivre l'évolution : 


\section{B. Annexe 2 : Fiche d'interprétation du médecin nucléaire}

EXEMPLAIRE A REMPLIR PAR UN MEDECIN NUCLEAIRE SENIOR : Dr. ET A CONSERVER AU SECRETARIAT DANS LA POCHETTE DEDIEE.

QUESTIONNAIRE D'EVALUATION DE L'APPORT DIAGNOSTIC DU TEP CEREBRAL 18 F-DOPA AUPRES DES MEDECINS NEUROLOGUES PRESCRIPTEURS

Nom et Prénom du Patient :.

Sexe :

IPP :

Date de naissance

Age

Prescripteur : $\mathrm{Dr}$

Prise de Carbidopa : $\square$ oui $\square$ non

Début de la symptomatologie : $\quad \square<6$ mois

6 mois - 2 ans

$\square 2$ ans -5 ans $\square>5$ ans

Latéralisation de la symptomatologie motrice :

$\square$ Droite unilatérale $\quad \square$ Gauche unilatérale $\quad \square$ Bilatérale G>D $\quad \square$ Bilatérale $D>G$

$\square$ Bilatérale symétrique

Résultat de la TEP :

Examen normal

$\square$ Douteux $\quad \square \quad$ Non contributif

$\square$ Ininterprétable, cause :

$\square$ Atteinte dopaminergique : $\square$ gauche

$\square$ droite

$\square$ bilatérale

$\square$ Autre

\section{A/Mesures: SUV en bw}

\begin{tabular}{|c|c|c|c|c|c|c|c|c|c|}
\hline & \multicolumn{4}{|c|}{ VOI à droite } & \multicolumn{4}{|c|}{ VOI à gauche } & \\
\hline & $\begin{array}{l}\text { Striatum } \\
\text { droit }\end{array}$ & $\begin{array}{l}\text { Noyau } \\
\text { caudé }\end{array}$ & $\begin{array}{l}\text { Putamen } \\
\text { ant }\end{array}$ & $\begin{array}{l}\text { Putamen } \\
\text { post }\end{array}$ & $\begin{array}{l}\text { Striatum } \\
\text { gauche }\end{array}$ & $\begin{array}{l}\text { Noyau } \\
\text { caudé }\end{array}$ & $\begin{array}{l}\text { Putamen } \\
\text { ant }\end{array}$ & $\frac{\text { Putamen }}{\text { post }}$ & $\begin{array}{l}\text { VOI } \\
\text { occip }\end{array}$ \\
\hline SUVmax & & & & & & & & & \\
\hline
\end{tabular}

Exemple de mesure: - Chaque sphère doit mesurer $2 \mathrm{~cm} 3$

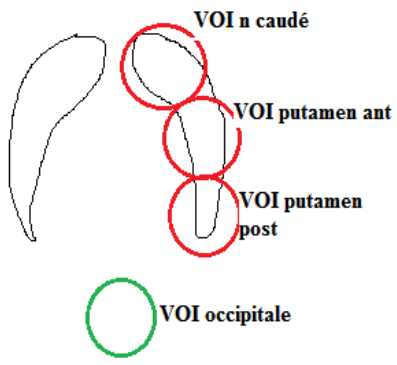

- Le VOI striatal est manuel, isocontour avec seuil arbitraire défini par l'observateur de telle manière qu'il englobe la totalité du striatum.

B/Evaluation visuelle: $\quad \square$ Type 1 : normal $\quad \square$ Type 2 : fixation asymétrique des putamens $\square$ Type 3 : fixation caudée exclusive $\quad \square$ Type 4 : fixation caudée exclusive très faible avec bruit de fond très élevé 


\section{Table des illustrations}

Figure $1:$ L tyrosine, L DOPA et Dopamine___ 26

Figure 2 : Aire tegmentale ventrale _ـ 27

Figure 3 : Schéma du cerveau en coupe coronale et des noyaux gris centraux___ 27

Figure 4 : Coupes de mésencéphale humain humain illustrant la dépigmentation de la substance noire

chez un sujet parkinsonien (Images de l'INCR : institut des neurosciences cliniques de Rennes)___ 29

Figure 5 : Marquage par un anticorps anti-ubiquitine révélant la présence de corps de Lewy au sein des neurones de la substance noire.

Figure 6 Représentation schématique d'une synapse dopaminergique : LAT= large aminoacid transporter, $D D C$ = dopa-décarboxylase, VMAT2 = type 2 vesicular monoamine transporter, DAT = dopamine transporter. Schéma adapté de (7) avec permission. 30 Figure 7 : Images de TEP cérébrale à la 18F-FDOPA en coupe axiale passant par les striatum réaxée plan CA-CP.

Figure 8 : Réalisation des VOI en coupe axiale plan CA-CP et sagittale ré axée dans le plan du putamen puis du noyau caudé. Sphère jaune : VOI striatal. Sphère grise: VOI noyau caudé. Sphère verte: VOI putamen antérieur. Sphère orange: VOI putamen postérieur. Sphère blanche: VOI occipitale. ___ 51 Figure 9 : Réponses au questionnaire __ 54 Figure 10: Taux de réponse par médecin__ 55

Figure $11:$ Hypothèse diagnostique avant TEP___ 58

Figure 12 : Diagnostic retenu après TEP___ 58

Figure 13 : Aide au diagnostic de la TEP cérébrale à la 18 F-FDOPA___ 64

Figure 14 : Evaluation du changement de prise en charge du patient après la TEP cérébrale à la 18 FFDOPA.

Figure 15: Type de changement de prise en charge après la TEP cérébrale à la 18 F-FDOPA___ 66

Figure 16: Patients inclus dans l'étude __ 69

Figure 17: Comparaison des valeurs de SUV avec et sans prise de carbidopa___ 71

Figure 18 : Boite à moustache SUV controlatéraux (atteint) et homolatéraux (atteint_moins) à l'atteinte

motrice prédominante chez les patients normaux et anormaux. __ 75

Figure 19 : Boite à moustache des rapports de fixation controlatéraux (atteint) et homolatéraux

(atteint_moins) à l'atteinte motrice prédominante chez les patients normaux et anormaux.__ 75

Figure 20 : Courbes ROC des SUV controlatéraux à l'atteinte motrice prédominante.__ 76

Figure 21 : Courbes ROC des SUV ipsilatéraux à l'atteinte motrice prédominante.___ 78

Figure 22 : Courbes ROC: SOR, COR, POR antérieur, POR postérieur sur le côté controlatéral à l'atteinte

motrice prédominante.

Figure 23 : Courbes ROC : SOR, COR, POR antérieur, POR postérieur sur le côté homolatéral à l'atteinte

motrice prédominante. 


\section{Table des tableaux}

Tableau 1 : données de la littérature sur l'apport diagnostic de la TEP cérébrale à la 18 F-FDOPA dans les syndromes parkinsoniens.

Tableau 2 : Données de la littérature sur le diagnostic différentiel des syndromes parkinsoniens par la TEP cérébrale à la 18F-FDOPA.

Tableau 3 : Paramètres d'acquisitions, de reconstructions et d'affichage des images TEP/TDM............... 49

Tableau 4 : Description de la population .................................................................................................. 56

Tableau 5 : hypothèses diagnostiques initiales et diagnostic final retenu pour les 91 cas. ......................... 64

Tableau 6 : Relecture : coefficients de corrélation intra-classe et intervalle de confiance pour l'ensemble

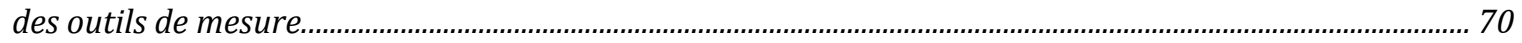

Tableau 7 : Moyenne des SUV et des rapports de fixation (SOR, COR, POR antérieur et postérieur).......... 72

Tableau 8 : SUV et rapports de fixation moyen chez les patients anormaux $(n=38)$ selon le côté de

l'atteinte motrice.

Tableau 9 : Sensibilité et spécificité des différentes figures de mérite du côté controlatéral à l'atteinte

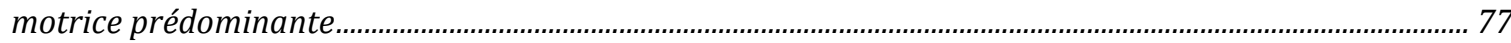

Tableau 10 : Aires sous la courbe des courbes ROC controlatérales à l'atteinte motrice............................. 77

Tableau 11 Sensibilité et spécificité des différentes figures de mérite du côté ipsilatéral à l'atteinte

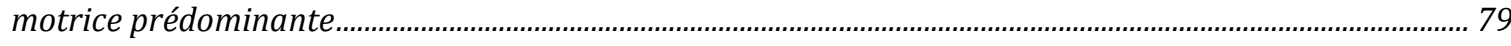

Tableau 12 : Aires sous la courbe des courbes ROC ipsilatérales à l'atteinte motrice. ................................. 79

Tableau 13 : Sensibilité et spécificité des différents outils de mesure du côté controlatéral à l'atteinte

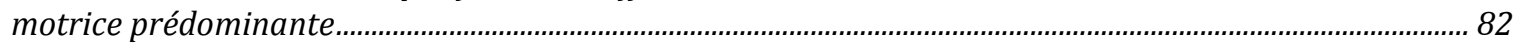

Tableau 14 : Aires sous la courbe des courbes ROC controlatérales à l'atteinte motrice. ............................. 82

Tableau 15 : Sensibilité et spécificité des différents outils de mesure du côté homolatéral à l'atteinte motrice prédominante

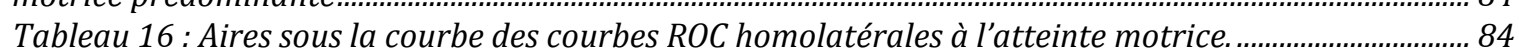

Tableau 17 : Récapitulatif des outils de mesure diagnostiques .......................................................................8 86 


\section{RÉSUMÉ}

Introduction : La maladie de Parkinson est la seconde maladie neuro-dégénérative la plus fréquente après la maladie d'Alzheimer. Si l'exploration de l'activité dopaminergique striatale reste dominée par la tomoscintigraphie des transporteurs pré-synaptiques de la dopamine, les données de notre revue de la littérature montrent que la TEP cérébrale à la 18F-FDOPA (TEP-DOPA) obtient des performances diagnostiques au moins équivalentes. L'analyse visuelle est le principal outil d'évaluation de la TEP-DOPA et l'analyse semi-quantitative permettrait de pallier son manque de reproductibilité. L'objectif principal de notre étude clinique monocentrique prospective était d'évaluer l'utilité clinique de la TEPDOPA dans les syndromes parkinsoniens par un questionnaire. L'objectif secondaire était de définir des outils diagnostiques semi-quantitatifs fiables et utilisables en routine clinique.

Matériel et méthodes: Du 15 octobre 2014 au 29 avril 2015 les médecins neurologues ont reçu un questionnaire pour chaque examen prescrit $(n=192)$. Une interprétation visuelle et semi-quantitative par un médecin nucléaire sénior a été réalisée. La fixation de la 18F-FDOPA a été quantifiée à partir des mesures de SUV mesurés sur plusieurs VOI et des rapports de fixation spécifique sur non spécifique (VOI occipital) ont été établis pour chaque VOI. Les paramètres diagnostiques de chaque SUV et rapport de fixation ont été étudiés par le biais de courbe ROC (AUC, index de Youden, sensibilité et spécificité).

Résultats : Nous avons obtenu 91 réponses pour les examens de 91 patients, d'un âge moyen de 71,4 ans. La TEP a permis de changer le diagnostic retenu dans $1 / 3$ des cas lorsqu'une MP était suspectée et dans 1 cas sur 2 lorsqu'un autre syndrome extra-pyramidal était suspecté. Selon les neurologues, la TEP a renforcé leur confiance dans leur diagnostic et a confirmé leur diagnostic dans $\mathbf{7 1 , 4 \%}$ des cas. Dans $\mathbf{5 7 \%}$ des cas la TEP a induit de façon certaine un changement de prise en charge du patient correspondant à un changement de traitement dans $\mathbf{7 3 \%}$ des cas. Il n'y avait pas de différence significative entre les SUV des patients normaux selon l'âge, le sexe ou le côté. Les courbes ROC ont été réalisées sur l'ensemble des SUV et rapports de fixation du côté homo et controlatéral à l'atteinte motrice prédominante. Le paramètre le plus robuste obtenant les meilleurs paramètres diagnostiques était le POR sur le putamen postérieur avec un seuil de 2,44 , on obtenait une sensibilité de 83,3\% (IC95\%: 68,6-93), une spécificité de 87,8\% (IC95\% : 75,2-95,4), une aire sous la courbe de 0,888 (index de Youden à 0,7109).

Conclusion : Selon notre étude la TEP-DOPA joue un rôle important dans la prise en charge des patients parkinsoniens tant au niveau diagnostique que clinique. Nous avons montré que l'analyse semi-quantitative est fiable, présente de bonnes performances diagnostiques et est réalisable en routine en complément de l'analyse visuelle. L'utilisation de logiciel d'analyse automatique est une perspective à explorer pour l'avenir. 


\section{ABSTRACT}

Introduction. Parkinson disease (PD) is the second neurodegenerative disease after Alzheimer's disease. Dopaminergic striatal assessment is currently performed by single photon emission tomography of the DAT transporter. We reviewed the literature and demonstrated that brain 18F-FDOPA brain PET was able to provide equivalent diagnostic performances. Visual analysis is the standard for 18F-FDOPA image analysis. However, semi-quantitative measurements could increase the reproducibility of the studies. The principal goal of our monocentric prospective study was to evaluate the clinical usefulness of brain 18F-FDOPA PET in the assesment of parkinsonian syndromes using a questionnaire. The second goal was to test a semi-quantitative approach in clinical routine.

Materials and methods. From October $15^{\text {th }} 2014$ to April 29th 2015, a questionnaire was sent to each referring neurologist for each 18F-FDOPA study they requested. Visual reading and semi-quantitative analysis were performed by a senior nuclear physician. 18F-FDOPA striatal uptake was quantified using VOI SUVs measures and by specific to non-specific ratios (to occipital uptake). Performances were evaluated using ROC analysis (AUC, Youden index, sensitivity and specificity).

Results. We obtained 91 answers to our questionnaires concerning 91 patients with a mean age of 71.4 years. PET results changed the diagnosis in $1 / 3$ of the cases when PD was suspected and in half of them when another extra-pyramidal syndrome was suspected. According to the referring neurologists, PET results increased their diagnosis confidence in $74.4 \%$ of the cases. In $57 \%$ of the cases, PET results induced a change in patient management corresponding to a treatment change in $73 \%$ of the cases. There was no significant difference in normal patients with age, sex and side. ROC analyses showed that the most robust figure of merit for the diagnosis was the POR measured on the contro-lateral posterior putamen. With a threshold of 2.44, sensitivity was 83.3\% (IC95\%: 68.6-93) and specificity 87.8\% (IC95\%: 75.2-95.4) with and AUC of 0.888 (Youden index of 0.7109).

Conclusion. Our results show that 18F-FDOPA PET plays an important role in parkinsonian patients' management. We also showed that semi-quantitative analysis was feasible in clinical practice with high diagnostic performances allowing coping with the lack of reproducibility of visual analysis which is not inferior. The use of automatic softwares could be useful in the future. 


\section{SERMENT D'HIPPOCRATE}

Au moment d'être admis à exercer la médecine, je promets et je jure d'être fidèle aux lois de l'honneur et de la probité.

Mon premier souci sera de rétablir, de préserver ou de promouvoir la santé dans tous ses éléments, physiques et mentaux, individuels et sociaux.

Je respecterai toutes les personnes, leur autonomie et leur volonté, sans aucune discrimination selon leur état ou leurs convictions. J'interviendrai pour les protéger si elles sont affaiblies, vulnérables ou menacées dans leur intégrité ou leur dignité. Même sous la contrainte, je ne ferai pas usage de mes connaissances contre les lois de l'humanité.

J'informerai les patients des décisions envisagées, de leurs raisons et de leurs conséquences. Je ne tromperai jamais leur confiance et n'exploiterai pas le pouvoir hérité des circonstances pour forcer les consciences.

Je donnerai mes soins à l'indigent et à quiconque me le demandera. Je ne me laisserai pas influencer par la soif du gain ou la recherche de la gloire.

Admis dans l'intimité des personnes, je tairai les secrets qui me seront confiés. Reçu à l'intérieur des maisons, je respecterai les secrets des foyers et ma conduite ne servira pas à corrompre les mours.

Je ferai tout pour soulager les souffrances. Je ne prolongerai pas abusivement les agonies. Je ne provoquerai jamais la mort délibérément.

Je préserverai l'indépendance nécessaire à l'accomplissement de ma mission. Je n'entreprendrai rien qui dépasse mes compétences. Je les entretiendrai et les perfectionnerai pour assurer au mieux les services qui me seront demandés.

J'apporterai mon aide à mes confrères ainsi qu'à leurs familles dans l'adversité.

Que les hommes et mes confrères m'accordent leur estime si je suis fidèle à mes promesses ; que je sois déshonorée et méprisée si j'y manque. » 